\title{
Quantum mechanics of Proca fields
}

Farhad Zamani' and Ali Mostafazadeh'

Citation: Journal of Mathematical Physics 50, 052302 (2009); doi: 10.1063/1.3116164

View online: http://dx.doi.org/10.1063/1.3116164

View Table of Contents: http://aip.scitation.org/toc/jmp/50/5

Published by the American Institute of Physics

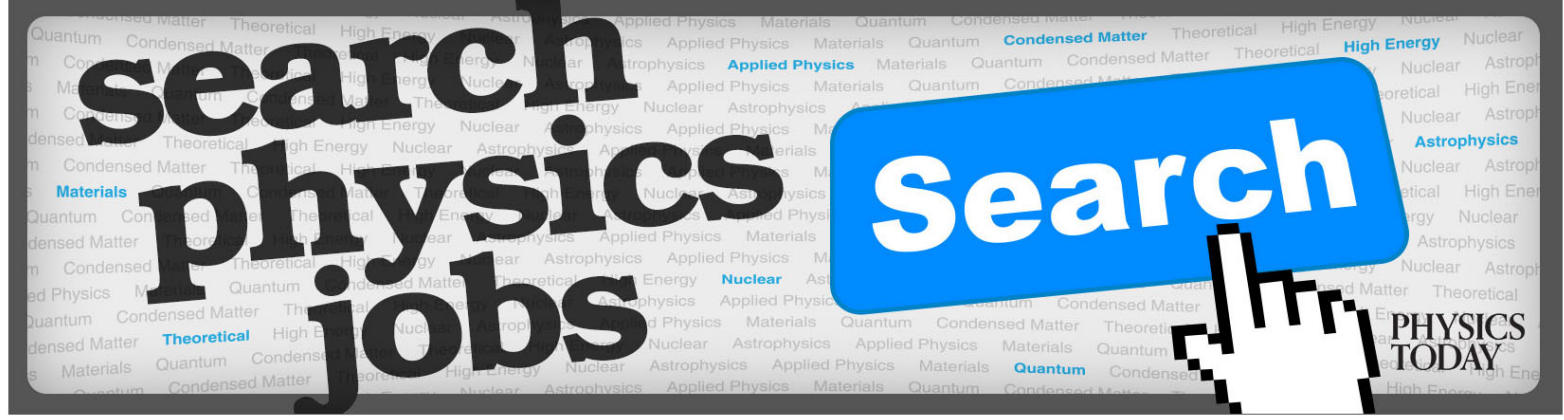




\title{
Quantum mechanics of Proca fields
}

\author{
Farhad Zamani ${ }^{1, a)}$ and Ali Mostafazadeh ${ }^{2, b)}$ \\ ${ }^{1}$ Department of Physics, Institute for Advanced Studies in Basic Sciences, Zanjan 45195- \\ 1159, Iran \\ ${ }^{2}$ Department of Mathematics, Koç University, Rumelifeneri Yolu, Sariyer, Istanbul 34450, \\ Turkey
}

(Received 8 December 2008; accepted 17 March 2009; published online 14 May 2009)

\begin{abstract}
We construct the most general physically admissible positive-definite inner product on the space of Proca fields. Up to a trivial scaling this defines a five-parameter family of Lorentz invariant inner products that we use to construct a genuine Hilbert space for the quantum mechanics of Proca fields. If we identify the generator of time translations with the Hamiltonian, we obtain a unitary quantum system that describes first-quantized Proca fields and does not involve the conventional restriction to the positive-frequency fields. We provide a rather comprehensive analysis of this system. In particular, we examine the conserved current density responsible for the conservation of the probabilities, explore the global gauge symmetry underlying the conservation of the probabilities, obtain a probability current density, construct position, momentum, helicity, spin, and angular momentum operators, and determine the localized Proca fields. We also compute the generalized parity $(\mathcal{P})$, generalized time-reversal $(\mathcal{T})$, and generalized charge or chirality $(\mathcal{C})$ operators for this system and offer a physical interpretation for its $\mathcal{P} \mathcal{T}_{-}, \mathcal{C}_{-}$, and $\mathcal{C P}$-symmetries. (C) 2009 American Institute of Physics.
\end{abstract}

[DOI: $10.1063 / 1.3116164]$

\section{INTRODUCTION}

Quantum mechanics (QM) and special relativity (SR) constitute the basis for the main body of modern physics developed during the first half of the 20th century. The conceptual marriage of QM and SR is relativistic QM (RQM) in which the one-particle quantum wave functions are identified with the solutions of an appropriate field equation. The solution space of these equations provides the representation (Hilbert) spaces for the irreducible (projective) representations of the Poincaré group that in turn define the elementary particles. ${ }^{1-3}$ One can construct the Fock space associated with such a Hilbert space and define the quantum field operators as operators acting in this space. In this sense, RQM provides a basis for quantum field theory (QFT). Although a fully relativistic treatment of elementary particles requires the methods of QFT, there are processes and energy scales where one can safely employ RQM. This is, for example, the case in the relativistic treatment of quantum systems displaying quantum superposition and quantum entanglement. ${ }^{4}$

Since the early days of RQM the formulation of a consistent (first-quantized) quantum theory with a genuine probabilistic interpretation for bosonic fields has encountered severe difficulties. It was in an attempt at such a formulation for the massive scalar [Klein-Gordon (KG)] fields that Dirac introduced the electron's wave equation and discovered the antimatter. His proposal that led to the development of second-quantized field theories was also mainly motivated by this problem. Recently, we used the methods of pseudo-Hermitian $\mathrm{QM}^{5}$ (PHQM) to give a complete formulation

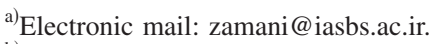

${ }^{b}$ Electronic mail: amostafazadeh@ku.edu.tr.
} 
of a genuine quantum mechanical treatment of $\mathrm{KG}$ fields. ${ }^{6}$ The purpose of the present paper is to employ a similar approach to devise a consistent quantum theory of free first-quantized massive vector fields.

Research on the theory of massive vector fields started with the work of Proca ${ }^{7}$ whose original aim was to obtain a description of the four states of electron-positron system using a four-vector. Proca attempted to pursue the approach of Pauli and Weisskopf ${ }^{8}$ who had quantized the KG fields and interpreted the KG conserved current with that of the electric charge rather than the probability. Although Proca's results did not serve its original purpose, its mathematical formalism could be used to treat massive vector fields.

In its manifestly covariant form, Proca's equation for a free massive vector field $A^{\mu}$ reads $^{7}$

$$
\partial_{\mu} F^{\mu \nu}-\mathcal{M}^{2} A^{\nu}=0
$$

where

$$
F^{\mu \nu}:=\partial^{\mu} A^{\nu}-\partial^{\nu} A^{\mu},
$$

$\mathcal{M}:=m c / \hbar$ is the inverse of the Compton's wavelength, $m \in \mathrm{R}^{+}$is the mass, $\partial_{\mu} \partial^{\mu}:=\eta^{\mu \nu} \partial_{\mu} \partial_{\nu}$, and $\left(\eta^{\mu \nu}\right)=\left(\eta_{\mu \nu}\right)^{-1}:=\operatorname{diag}(-1,1,1,1)$.

If we apply $\partial_{\nu}$ to (1) and use $F_{\mu \nu}=-F_{\nu \mu}$, we find that $A^{\mu}$ satisfies the Lorentz condition,

$$
\partial_{\mu} A^{\mu}=0 .
$$

Making use of this relation in (1) we find

$$
\left(\partial_{\nu} \partial^{\nu}-\mathcal{M}^{2}\right) A^{\mu}=0 .
$$

This means that the four components of the vector field $A^{\mu}$ satisfy the KG equation. Note that the Proca equation does not have a gauge symmetry. Therefore (3) is not a gauge choice but rather a constraint that is to be imposed on $A^{\mu}$. In fact, it is not difficult to show that (4) together with (3) are equivalent to the Proca equation (1). Similarly to the case of KG fields, the presence of the second time derivative in (4) is responsible for the difficulties associated with devising a sound probabilistic interpretation for the Proca fields.

An important motivation for the study of Proca fields is their close relationship with electromagnetic fields. For example, the study of Proca fields may shed light on the interesting problem of the consequences of a nonzero photon rest mass which has been the subject of intensive research over the past several decades. (See the review article 9 in the list of references.) It might also be possible to construct a first-quantized quantum theory of a photon by taking the zero-mass limit of that of a Proca field. This can, in particular, led to a resolution of the important issue of the construction of an appropriate position operator and localized states for a photon. ${ }^{10}$

The literature on the Proca fields is quite extensive. There are a number of publications that deal with the issue of the consistency of Proca's theory and the difficulties associated with interacting massive vector bosons (see Refs. 11 and 12 and references therein). It turns out that there are various ways of formulating a relativistic wave equation describing the dynamical states of a massive vector boson. The famous ones employ the equations of Proca, Duffin-Kemmer-Petiau (DKP), ${ }^{13}$ and Weinberg-Shay-Good. ${ }^{14}$ These are equivalent in the absence of interactions but lead to different predictions upon the inclusion of interactions.

Among the works that are directly relevant to the subject of the present article are those of Taketani and Sakata, ${ }^{15}$ Case, ${ }^{16}$ and Foldy. ${ }^{17}$ Using the analogy with the Maxwell equations, Taketani and Sakata ${ }^{15}$ reduced the ten-component DKP wave function to a six-component wave function satisfying a Schrödinger equation. They made use of this representation to study the interaction of the field with an electromagnetic field. Employing the six-component TaketaniSakata (TS) representation, Case ${ }^{16}$ cast the Proca equation into a form in which the positive- and negative-energy states were separately described by three-component wave functions. The latter is the so-called Foldy's canonical representation ${ }^{17}$ which Case used to study the nonrelativistic limit of the Proca equation and obtained the position and spin operators acting on the TS wave func- 
tions. The approaches of Case ${ }^{16}$ and Foldy ${ }^{17}$ involve the use of an indefinite inner product ${ }^{18}$ on the space of six-component TS wave functions. This seems to be the main reason why these authors did not suggest any reasonable solution for the problem of the probabilistic interpretation of their theories. The problem of the construction of an appropriate position operator and the corresponding localized states for Proca fields has also been considered by various authors, e.g., Refs. 19-22. But a universally accepted solution has not been given. Some other more recent articles on Proca fields are Refs. 23 and 24.

In Refs. 25-27, 6, and 28, we have employed the results obtained within the framework of PHQM to formulate a consistent QM of KG fields. Here we pursue a similar approach to treat the Proca fields. The first step in this direction has been taken by Jakubský and Smejkal ${ }^{29}$ who constructed a one-parameter family of admissible inner products on the space of Proca fields. In what follows we will offer a more systematic and general treatment of this problem. In particular, we give a complete characterization of Lorentz-invariant positive-definite inner products that render the generator of the time translations and the helicity operator self-adjoint. We further construct a position operator and the corresponding localized states for the Proca fields and examine a variety of related problems.

In the remainder of this section we briefly review PHQM and give the notations and conventions that we use throughout the paper.

$\mathrm{PHQM}^{5,30}$ has developed in an attempt to give a mathematically consistent formulation for the $P T$-symmetric QM. ${ }^{31}$ In PHQM, a quantum system is described by a diagonalizable Hamiltonian operator $H$ that acts in an auxiliary Hilbert space $\mathcal{H}^{\prime}$ and has a real spectrum. One can show (under some general conditions) that these conditions imply that $H$ is Hermitian with respect to a positive-definite inner product that is generally different from the defining inner product $\langle\cdot, \cdot\rangle$ of $\mathcal{H}^{\prime}{ }^{32}$ This motivates the following definition of a pseudo-Hermitian operator: ${ }^{33} \mathrm{H}$ is a pseudoHermitian operator if there is a linear, invertible, Hermitian (metric) operator $\eta: \mathcal{H}^{\prime} \rightarrow \mathcal{H}^{\prime}$ satisfying $H^{\dagger}=\eta H \eta^{-1}$. This condition is equivalent to the requirement that $H$ be self-adjoint with respect to the (possibly indefinite) inner product $\langle\langle\cdot, \cdot\rangle\rangle_{\eta}:=\langle\cdot, \eta \cdot\rangle$. It turns out that for a given pseudo-Hermitian operator $H$, the metric operator $\eta$ is not unique. If we make a particular choice for $\eta$, we say that $H$ is $\eta$-pseudo-Hermitian.

A proper subset of pseudo-Hermitian operators is the set of quasi-Hermitian operators. ${ }^{34} \mathrm{~A}$ quasi-Hermitian operator $H$ is a pseudo-Hermitian operator that is $\eta_{+}$-pseudo-Hermitian for a positive-definite metric operator $\eta_{+}$, i.e.,

$$
H^{\dagger}=\eta_{+} H \eta_{+}^{-1} .
$$

This means that $H$ is Hermitian (self-adjoint) with respect to the positive-definite inner product,

$$
\langle\langle\cdot, \cdot\rangle\rangle_{\eta_{+}}:=\left\langle\cdot, \eta_{+} \cdot\right\rangle .
$$

In particular, $H$ is a diagonalizable operator with a real spectrum and can be mapped to a Hermitian operator via a similarity transformation. ${ }^{32}$

A quasi-Hermitian Hamiltonian operator defines a quantum system with a consistent probabilistic interpretation provided that one constructs the physical Hilbert space $\mathcal{H}$ of the system using the inner product $\langle\langle\cdot, \cdot\rangle\rangle_{\eta_{+}}$and identifies the observables of the theory with the self-adjoint operators acting in $\mathcal{H}$ (Refs. 34 and 5) $(\mathcal{H}$ is the Cauchy completion of the inner product space obtained by endowing the span of the eigenvector of $H$ (in $\mathcal{H}^{\prime}$ ) with the inner product $\langle\langle\cdot, \cdot\rangle\rangle_{\eta_{+}}$)

Although PHQM employs quasi-Hermitian Hamiltonian operators, the study of the pseudoHermitian Hamiltonians has proven to be essential in understanding the role of antilinear symmetries such as $P T$. As shown in Ref. 35, given a diagonalizable pseudo-Hermitian Hamiltonian $H$, one may introduce generalized parity $(\mathcal{P})$, time-reversal $(\mathcal{T})$, and charge or chirality $(\mathcal{C})$ operators and establish the $\mathcal{C}$-, $\mathcal{P} \mathcal{T}$-, and $\mathcal{C P} \mathcal{T}$-symmetries of $H$ that would, respectively, generalize $C, P T$, and $C P T$ symmetries $^{36}$ of $P T$-symmetric QM. (See also Ref. 37.) We recall that $H$ is $\mathcal{P}$-pseudo-Hermitian and $\mathcal{T}$-anti-pseudo-Hermitian, $\mathcal{P}$ and $\mathcal{T}$ are, respectively, linear and antilinear 
invertible operators, and $\mathcal{C}$ is a linear involution $\left(\mathcal{C}^{2}=1\right)$ satisfying $\mathcal{C}=\eta_{+}^{-1} \mathcal{P}$ (Ref. 35). (A simple consequence of the latter relation is that the $C P T$-inner product of Ref. 36 is a particular example of the inner products (6) of Refs. 32, 38, and 39.)

Next, we give our notations and conventions.

Throughout this paper $a=\left(a^{0}, \mathbf{a}\right)$ stands for a four-vector $a^{\mu}$, Greek indices take on the values $0,1,2,3$, Latin indices take on 1,2,3, and $\varepsilon_{i j k}$ denotes the totally antisymmetric Levi-Civita symbol with $\varepsilon_{123}=1$. We employ Einstein's summation convention over repeated indices and use $\sigma_{0}$ and $\lambda_{0}$ to denote the $2 \times 2$ and $3 \times 3$ identity matrices, respectively. Recall that $\sigma_{0}$ together with the Pauli matrices

$$
\sigma_{1}=\left(\begin{array}{ll}
0 & 1 \\
1 & 0
\end{array}\right), \quad \sigma_{2}=\left(\begin{array}{cc}
0 & -i \\
i & 0
\end{array}\right), \quad \sigma_{3}=\left(\begin{array}{cc}
1 & 0 \\
0 & -1
\end{array}\right)
$$

form a basis of the vector space of $2 \times 2$ complex matrices, and that $\left\{\sigma_{0}, \sigma_{3}\right\}$ is a maximal set of commuting matrices. We denote their common eigenvectors by $e_{+}:=\left(\begin{array}{c}1 \\ 0\end{array}\right)$ and $e_{-}:=\left(\begin{array}{l}0 \\ 1\end{array}\right)$, where the labels correspond to the eigenvalues of $\sigma_{3}$.

Similarly, $\lambda_{0}$ together with

$$
\begin{array}{cc}
\lambda_{1}=\left(\begin{array}{lll}
0 & 1 & 0 \\
1 & 0 & 0 \\
0 & 0 & 0
\end{array}\right), & \lambda_{2}=\left(\begin{array}{ccc}
0 & -i & 0 \\
i & 0 & 0 \\
0 & 0 & 0
\end{array}\right), \quad \lambda_{3}=\left(\begin{array}{ccc}
1 & 0 & 0 \\
0 & -1 & 0 \\
0 & 0 & 0
\end{array}\right), \quad \lambda_{4}=\left(\begin{array}{ccc}
0 & 0 & 1 \\
0 & 0 & 0 \\
1 & 0 & 0
\end{array}\right), \\
\lambda_{5}=\left(\begin{array}{ccc}
0 & 0 & -i \\
0 & 0 & 0 \\
i & 0 & 0
\end{array}\right), & \lambda_{6}=\left(\begin{array}{ccc}
0 & 0 & 0 \\
0 & 0 & 1 \\
0 & 1 & 0
\end{array}\right), \quad \lambda_{7}=\left(\begin{array}{ccc}
0 & 0 & 0 \\
0 & 0 & -i \\
0 & i & 0
\end{array}\right), \quad \lambda_{8}=\frac{1}{\sqrt{3}}\left(\begin{array}{ccc}
1 & 0 & 0 \\
0 & 1 & 0 \\
0 & 0 & -2
\end{array}\right)
\end{array}
$$

form a basis for the vector space of $3 \times 3$ complex matrices, and $\left\{\lambda_{0}, \lambda_{3}, \lambda_{8}\right\}$ is a set of maximally commuting matrices whose common eigenvectors we denote by

$$
e_{+1}:=\left(\begin{array}{l}
1 \\
0 \\
0
\end{array}\right), \quad e_{-1}:=\left(\begin{array}{l}
0 \\
1 \\
0
\end{array}\right), \quad e_{0}:=\left(\begin{array}{l}
0 \\
0 \\
1
\end{array}\right) .
$$

Again the labels 0 and \pm 1 are the eigenvalues of $\lambda_{3}$.

Using the bases $\left\{\sigma_{i}\right\}$ and $\left\{\lambda_{j}\right\}$ we can construct the basis $\left\{\Sigma_{\mathfrak{m}}\right\}$ for the vector space of $6 \times 6$ complex matrices, where for all $\mathfrak{m} \in\{0,1,2, \cdots, 35\}$

$$
\Sigma_{\mathfrak{m}}:=\sigma_{i} \otimes \lambda_{j}, \quad \text { if } \mathfrak{m}=i+4 j .
$$

The operators $\Sigma_{0}, \Sigma_{3}, \Sigma_{12}, \Sigma_{15}, \Sigma_{32}$, and $\Sigma_{35}$ form a maximal set of commuting operators with common eigenvectors,

$$
e_{\epsilon, s}:=e_{\epsilon} \otimes e_{s},
$$

where $\epsilon \in\{+,-\}$ and $s \in\{-1,0,+1\}$.

The organization of the paper is as follows. In Sec. II, we present a six-component formulation of the Proca equation, establish its relation with pseudo-Hermiticity, and construct a positivedefinite metric operator and the corresponding inner product. In Sec. III, we compute the generalized parity $\mathcal{P}$, time-reversal $\mathcal{T}$, and charge or chirality $\mathcal{C}$ operators and elaborate on the $\mathcal{C}-, \mathcal{P} \mathcal{T}$-, and $\mathcal{C P} \mathcal{T}$-symmetries of the Hamiltonian. In Sec. IV, we derive the expression for the most general physically admissible positive-definite inner product on the solution space of the Proca equation and demonstrate the unitary equivalence of the representation obtained by the corresponding Hilbert space and the generator of time translations as the Hamiltonian with the Foldy representation. In Sec. V, we obtain and explore the properties of a conserved current density that supports 
the conservation of the probabilities. In Sec. VI, we introduce a position basis and the associated position wave functions for the Proca fields. In Sec. VII, we discuss the position, spin, helicity, momentum, and angular momentum operators and construct the relativistic localized states for the Proca fields. In Sec. VIII, we compute the probability current density for the spatial localization of a Proca field. In Sec. IX, we study the gauge symmetry associated with the conservation of probabilities. Finally in Sec. X, we present a summary of our main results and discuss the differences between our approach of finding the most general admissible inner product and that of Ref. 29.

\section{PSEUDO-HERMITICITY AND PROCA FIELDS}

\section{A. Covariant dynamical field equation and helicity states}

To begin our investigation, we briefly formulate the covariant dynamical theory of Proca fields and review the physical polarization and helicity states.

Consider a Proca field $A^{\mu}$ such that for all $x^{0} \in \mathbb{R}$ and $\mu \in\{0,1,2,3\}, \int_{\mathbb{R}^{3}} d^{3} \mathbf{x}\left|A^{\mu}\left(x^{0}, \mathbf{x}\right)\right|^{2}<\infty$. Then we can express (4) as a dynamical equation in the Hilbert space $L^{2}\left(\mathbb{R}^{3}\right) \oplus L^{2}\left(\mathbb{R}^{3}\right) \oplus L^{2}\left(\mathbb{R}^{3}\right)$ $\oplus L^{2}\left(\mathbb{R}^{3}\right)$, namely,

$$
\ddot{A}\left(x^{0}\right)+D A\left(x^{0}\right)=0,
$$

where a dot denotes a $x^{0}$-derivative, for all $\mu \in\{0,1,2,3\}$ and $x^{0} \in \mathbb{R}$ the functions $A^{\mu}\left(x^{0}\right): \mathbb{R}^{3}$ $\rightarrow \mathrm{C}$ are defined by

$$
A^{\mu}\left(x^{0}\right)(\mathbf{x}):=A^{\mu}\left(x^{0}, \mathbf{x}\right), \quad \forall \mathbf{x} \in \mathbb{R}^{3},
$$

and $D: L^{2}\left(\mathbb{R}^{3}\right) \rightarrow L^{2}\left(\mathbb{R}^{3}\right)$ is the Hermitian operator,

$$
[D f](\mathbf{x}):=\left[\mathcal{M}^{2}-\nabla^{2}\right] f(\mathbf{x}), \quad \forall f \in L^{2}\left(\mathbb{R}^{3}\right) .
$$

Clearly, for all $\mu \in\{0,1,2,3\}$ and $x^{0} \in \mathbb{R}, A^{\mu}\left(x^{0}\right)$ belongs to $L^{2}\left(\mathbb{R}^{3}\right)$, and $D$ is a positive-definite operator with eigenvalues $\omega_{\mathbf{k}}^{2}:=k^{2}+\mathcal{M}^{2}$ and the corresponding eigenvectors $\phi_{\mathbf{k}}(\mathbf{x}):=(2 \pi)^{-3 / 2} e^{i \mathbf{k} \cdot \mathbf{x}}$ $=\langle\mathbf{x} \mid \mathbf{k}\rangle$, where $\mathbf{k} \in \mathbb{R}^{3}, k:=|\mathbf{k}|$, and $\langle\cdot \mid \cdot\rangle$ denotes the inner product of $L^{2}\left(\mathbb{R}^{3}\right)$.

It is important to observe that a solution of (11) is a Proca field provided that for all $x^{0} \in \mathbb{R}$ the functions $A\left(x^{0}\right)$ fulfill the Lorentz condition that we can express as the constraint,

$$
\mathfrak{L}\left[A\left(x^{0}\right)\right]:=\dot{A}^{0}\left(x^{0}\right)+i \mathfrak{K} \cdot \mathbf{A}\left(x^{0}\right)=0,
$$

where for all $f \in L^{2}\left(\mathbb{R}^{3}\right),(\mathfrak{K} f)(\mathbf{x}):=-i \boldsymbol{\nabla} f(\mathbf{x})$, and "." is the usual dot product.

The Lorentz condition (13) implies that there are only three independent components of $A$; using this condition we can express $A^{0}\left(x^{0}\right)$ and $\dot{A}^{0}\left(x^{0}\right)$ in terms of $\mathbf{A}\left(x^{0}\right)$ and $\dot{\mathbf{A}}\left(x^{0}\right)$. This means that the initial data for the Proca equation (11) are given by $\left(\mathbf{A}\left(x_{0}^{0}\right), \dot{\mathbf{A}}\left(x_{0}^{0}\right)\right)$ for some initial value $x_{0}^{0}$ $\in R$ of $x_{0}$. We shall, however, employ a manifestly covariant approach and treat all components of the field $A\left(x^{0}\right)$ on an equal footing. As a result we define the complex vector space $(\mathcal{V})$ of solutions of the Proca equation (11) as

$$
\mathcal{V}:=\left\{A:=\left(A^{0}, \mathbf{A}\right) \mid \forall x^{0} \in \mathbb{R},\left[\partial_{0}^{2}+D\right] A^{\mu}\left(x^{0}\right)=0, \mathfrak{L}\left[A\left(x^{0}\right)\right]=0\right\} .
$$

It is not difficult to show that the Lorentz condition (13) may be imposed in the form of a constraint on the initial data for the dynamical equation (11), namely, (The $\ddot{A}\left(x_{0}^{0}\right)$ in $\mathfrak{L}\left[\dot{A}\left(x_{0}^{0}\right)\right]$ stands for $-D A\left(x_{0}^{0}\right)$.)

$$
\mathfrak{L}\left[A\left(x_{0}^{0}\right)\right]=\mathfrak{L}\left[\dot{A}\left(x_{0}^{0}\right)\right]=0 .
$$

This in turn implies 
$\mathcal{V}=\left\{A:=\left(A^{0}, \mathbf{A}\right) \mid \forall x^{0} \in \mathbb{R},\left[\partial_{0}^{2}+D\right] A^{\mu}\left(x^{0}\right)=0\right.$, and $\left.\exists x_{0}^{0} \in \mathbb{R}, \mathfrak{L}\left[A\left(x_{0}^{0}\right)\right]=\mathfrak{L}\left[\dot{A}\left(x_{0}^{0}\right)\right]=0\right\}$.

We can express the solution of (11) in terms of $\left(A^{\mu}\left(x_{0}^{0}\right), \dot{A}^{\mu}\left(x_{0}^{0}\right)\right)$ according to ${ }^{26,40}$

$$
A^{\mu}\left(x^{0}\right)=\cos \left[\left(x^{0}-x_{0}^{0}\right) D^{1 / 2}\right] A^{\mu}\left(x_{0}^{0}\right)+\sin \left[\left(x^{0}-x_{0}^{0}\right) D^{1 / 2}\right] D^{-1 / 2} \dot{A}^{\mu}\left(x_{0}^{0}\right) .
$$

Note that here and throughout this paper we use the spectral resolution of $D$ to define its powers, $D^{\alpha}:=\int_{\mathbb{R}^{3}} d^{3} \mathbf{k}\left(k^{2}+\mathcal{M}^{2}\right)^{\alpha}|\mathbf{k}\rangle\langle\mathbf{k}|$ for all $\alpha \in \mathbb{R}$.

Next, consider the plane-wave solutions of (4),

$$
A_{\epsilon}^{\mu}(\mathbf{k}, \sigma ; x)=N_{\epsilon, \mathbf{k}} a_{\epsilon}^{\mu}(\mathbf{k}, \sigma) e^{-i \epsilon \omega_{\mathbf{k}} x^{0}} \phi_{\mathbf{k}}(\mathbf{x})
$$

where $N_{\epsilon, \mathbf{k}}$ are normalization constants (Lorentz scalars) and $a_{\epsilon}^{\mu}(\mathbf{k}, \sigma)$ denotes a set of normalized four-dimensional polarization vectors fulfilling the Lorentz condition, i.e.,

$$
a_{\epsilon \mu}(\mathbf{k}, \sigma) a_{\epsilon}^{\mu}\left(\mathbf{k}, \sigma^{\prime}\right)=\delta_{\sigma \sigma^{\prime}}, \quad k_{\mu} a_{\epsilon}^{\mu}(\mathbf{k}, \sigma)=0 .
$$

These relations show that the Proca field (18) has only three physical polarization states $(\sigma$ $\in\{1,2,3\})$. In a fixed reference frame in which the plane wave has momentum $\mathbf{k}$, we choose to work with a pair of transverse polarization vectors, 3,41

$$
a_{\epsilon}(\mathbf{k}, 1)=\left(0, \mathbf{a}_{\epsilon}(\mathbf{k}, 1)\right), \quad a_{\epsilon}(\mathbf{k}, 2)=\left(0, \mathbf{a}_{\epsilon}(\mathbf{k}, 2)\right),
$$

whose spacelike components, $\mathbf{a}_{\epsilon}(\mathbf{k}, \sigma)$ with $\sigma=1,2$, are normalized vectors perpendicular to $\mathbf{k}$. As a longitudinal polarization vector we choose ${ }^{3,41}$

$$
a_{\epsilon}(\mathbf{k}, 3)=\left(\frac{k}{\mathcal{M}}, \frac{k^{0}}{\mathcal{M}} \frac{\mathbf{k}}{k}\right), \quad k^{0}=\epsilon \omega_{\mathbf{k}},
$$

which together with (20) form an orthonormal set. These polarization vectors also fulfil the completeness relation, 3,41

$$
\sum_{\sigma=1}^{3} a_{\epsilon}^{\mu}(\mathbf{k}, \sigma) a_{\epsilon}^{\nu}(\mathbf{k}, \sigma)=\eta^{\mu \nu}+\frac{1}{\mathcal{M}^{2}} k^{\mu} k^{\nu}
$$

(The longitudinal polarization vector used in Ref. 29 is not normalized. Note also that, unlike the authors of Ref. 29, we do not fix the transverse polarization vectors $a_{\epsilon}(\mathbf{k}, \sigma), \sigma=1,2$. Although we fix a reference frame in which their timelike components are zero, their spacelike components are still arbitrary. Instead of fixing these vectors, we only fix the longitudinal polarization vector (21) and make use of Eqs. (19) and (22) to do the necessary calculations throughout this paper.)

It turns out that the use of the circular polarization vectors,

$$
u_{\epsilon, 0}(\mathbf{k}):=a_{\epsilon}(\mathbf{k}, 3), \quad u_{\epsilon, \pm 1}(\mathbf{k}):=\frac{1}{\sqrt{2}}\left[a_{\epsilon}(\mathbf{k}, 1) \pm i a_{\epsilon}(\mathbf{k}, 2)\right]
$$

simplifies many of the calculations. This is because their spatial components are eigenvectors of the helicity operator,

$$
\mathfrak{h}=\frac{\mathfrak{K} \cdot \mathbf{S}}{|\mathfrak{K}|}=\hat{\mathfrak{K}} \cdot \mathbf{S}=\frac{i}{|\mathfrak{K}|}\left(\begin{array}{ccc}
0 & -\mathfrak{K}_{3} & \mathfrak{K}_{2} \\
\mathfrak{K}_{3} & 0 & -\mathfrak{K}_{1} \\
-\mathfrak{K}_{2} & \mathfrak{K}_{1} & 0
\end{array}\right),
$$

where $\mathfrak{K}=:\left(\mathfrak{K}_{1}, \mathfrak{K}_{2}, \mathfrak{K}_{3}\right), \hat{\mathfrak{K}}:=\mathfrak{K} /|\mathfrak{K}|$, and 


$$
S_{1}:=\lambda_{7}, \quad S_{2}:=-\lambda_{5}, \quad S_{3}:=\lambda_{2}
$$

are the angular momentum operators in the spin-one representation of the rotation group. ${ }^{42}$ They satisfy

$$
S_{i} S_{j} S_{k}+S_{k} S_{j} S_{i}=\delta_{i j} S_{k}+\delta_{k j} S_{i}, \quad\left[S_{i}, S_{j}\right]=i \epsilon_{i j k} S_{k} .
$$

A simple consequence of these relations is the identity $\mathfrak{h}^{3}=\mathfrak{h}$ which we shall make use of in the sequel.

We can also construct the basic (plane wave) solutions of (4) that have a definite helicity. These are given by

$$
A_{\epsilon, h}^{\mu}\left(\mathbf{k} ; x^{0}, \mathbf{x}\right)=N_{\epsilon, h}(\mathbf{k}) u_{\epsilon, h}^{\mu}(\mathbf{k}) e^{-i \epsilon \omega_{\mathbf{k}} \mathbf{x}^{0}} \phi_{\mathbf{k}}(\mathbf{x}), \quad \epsilon= \pm, h=-1,0,1,
$$

where $N_{\epsilon, h}(\mathbf{k})$ are normalization constants.

\section{B. Six-component formulation and pseudo-Hermiticity}

In analogy with electromagnetism, we express the antisymmetric tensor $F^{\mu \nu}$ in terms of the $\mathbf{E}$ and $\mathbf{B}$ fields, ${ }^{43}$

$$
F^{i j}=: \epsilon^{i j m} B_{m}, \quad F^{0 i}=: E^{i} .
$$

Then the Proca equation (1) takes the form

$$
\begin{gathered}
\mathbf{B}=\boldsymbol{\nabla} \times \mathbf{A}, \quad \dot{\mathbf{A}}=-\mathbf{E}-\nabla A^{0}, \\
A^{0}=-\mathcal{M}^{-2} \nabla \cdot \mathbf{E}, \quad \dot{\mathbf{E}}=\mathcal{M}^{2} \mathbf{A}+\boldsymbol{\nabla} \times \mathbf{B} .
\end{gathered}
$$

Next, we let $\tilde{\mathcal{H}}:=\left\{\mathbf{Y}: \mathbb{R}^{3} \rightarrow \mathrm{C}^{3} \mid<\mathbf{Y}, \mathbf{Y}><\infty\right\}$ denote the Hilbert space of vector fields, where for all $\mathbf{Y}, \mathbf{Z}: \mathbb{R}^{3} \rightarrow \mathrm{C}^{3},\left\langle\mathbf{Y}, \mathbf{Z}>:=\int_{\mathbb{R}^{3}} d^{3} \mathbf{x} \mathbf{Y}(\mathbf{x})^{*} \cdot \mathbf{Z}(\mathbf{x})\right.$. If we eliminate $\mathbf{B}$ and $A^{0}$, we can express (29) as a set of dynamical equations in the Hilbert space $\tilde{\mathcal{H}}$, namely,

$$
\dot{\mathbf{A}}\left(x^{0}\right)=-\mathbf{E}\left(x^{0}\right)+\mathcal{M}^{-2} \mathfrak{D} \mathbf{E}\left(x^{0}\right), \quad \dot{\mathbf{E}}\left(x^{0}\right)=D \mathbf{A}\left(x^{0}\right)+\mathfrak{D A}\left(x^{0}\right),
$$

where $\mathfrak{D}: \widetilde{\mathcal{H}} \rightarrow \widetilde{\mathcal{H}}$ is the Hermitian operator,

$$
[\mathfrak{D} \mathbf{Y}](\mathbf{x}):=\left[\left[(\mathfrak{K} \cdot \mathbf{S})^{2}-\mathfrak{K}^{2}\right] \mathbf{Y}\right](\mathbf{x})=\boldsymbol{\nabla}(\boldsymbol{\nabla} \cdot \mathbf{Y}(\mathbf{x})),
$$

$\mathbf{Y} \in \tilde{\mathcal{H}}$ is arbitrary, and we have used the identities

$$
\left[\mathfrak{K}^{2} \mathbf{Y}\right](\mathbf{x})=-\nabla^{2} \mathbf{Y}(\mathbf{x}), \quad[(\mathfrak{K} \cdot \mathbf{S}) \mathbf{Y}](\mathbf{x})=\nabla \times \mathbf{Y}(\mathbf{x}),
$$

which also imply

$$
\mathfrak{K} \cdot[(\mathfrak{K} \cdot \mathbf{S}) \mathbf{Y}]=0, \quad \mathfrak{K} \cdot\left[(\mathfrak{K} \cdot \mathbf{S})^{2} \mathbf{Y}\right]=0 .
$$

Next we express (30) as the Schrödinger equation (also known as the TS equation, ${ }^{15,16}$ )

$$
i \hbar \dot{\Psi}\left(x^{0}\right)=H \Psi\left(x^{0}\right),
$$

where for all $x^{0} \in \mathbb{R}$ the state vector $\Psi$ and the Hamiltonian $H$ are defined by

$$
\Psi\left(x^{0}\right):=\left(\begin{array}{l}
\mathbf{A}\left(x^{0}\right)-i \gamma \mathbf{E}\left(x^{0}\right) \\
\mathbf{A}\left(x^{0}\right)+i \gamma \mathbf{E}\left(x^{0}\right)
\end{array}\right),
$$




$$
H:=\frac{\hbar}{2}\left(\begin{array}{cc}
H_{1} & H_{2} \\
-H_{2} & -H_{1}
\end{array}\right),
$$

$\gamma \in \mathbb{R}-\{0\}$ is an arbitrary constant having the dimension of length and $H_{1}$ and $H_{2}$ are the following Hermitian operators that act in $\tilde{\mathcal{H}}$ :

$$
\begin{aligned}
& H_{1}:=\gamma(D+\mathfrak{D})+\gamma^{-1} \mathcal{M}^{-2}\left(\mathcal{M}^{2}-\mathfrak{D}\right)=\gamma\left(\mathcal{M}^{2}+(\mathfrak{K} \cdot \mathbf{S})^{2}\right)+\gamma^{-1} \mathcal{M}^{-2}\left(D-(\mathfrak{K} \cdot \mathbf{S})^{2}\right), \\
& H_{2}:=\gamma(D+\mathfrak{D})-\gamma^{-1} \mathcal{M}^{-2}\left(\mathcal{M}^{2}-\mathfrak{D}\right)=\gamma\left(\mathcal{M}^{2}+(\mathfrak{K} \cdot \mathbf{S})^{2}\right)-\gamma^{-1} \mathcal{M}^{-2}\left(D-(\mathfrak{K} \cdot \mathbf{S})^{2}\right) .
\end{aligned}
$$

Note that one can invert the first equation in (30) [or take a $x^{0}$-derivative of the second equation in (30) and use $\ddot{\mathbf{E}}=-D \mathbf{E}]$ to obtain

$$
\mathbf{E}\left(x^{0}\right)=-D^{-1}\left[\mathcal{M}^{2}+(\mathfrak{K} \cdot \mathbf{S})^{2}\right] \dot{\mathbf{A}}\left(x^{0}\right) .
$$

In view of (35) and (39), the six-component state vector $\Psi$ is completely determined in terms of $\mathbf{A}$ and $\dot{\mathbf{A}}$. This means that the space of the state vectors $\Psi$ is isomorphic (as a vector space) to the space of the initial conditions $\left(\mathbf{A}\left(x_{0}^{0}\right), \dot{\mathbf{A}}\left(x_{0}^{0}\right)\right)$. Because of the linearity of the Proca equation, the latter is also isomorphic to the vector space of Proca fields. (Similarly to the two-component representation of the KG fields, ${ }^{25}$ the choice of the six-component state vector (35) is not unique. Its general form is $\Psi_{g\left(x^{0}\right)}=g\left(x^{0}\right) \Psi$, where $g\left(x^{0}\right) \in G L(6, C)$. (30) is equivalent to the Schrödinger equation $i \hbar \dot{\Psi}_{g\left(x^{0}\right)}\left(x^{0}\right)=H_{g\left(x^{0}\right)} \Psi_{g\left(x^{0}\right)}\left(x^{0}\right)$, where $H_{g\left(x^{0}\right)}:=g\left(x^{0}\right) H g\left(x^{0}\right)^{-1}+i \hbar \dot{g}\left(x^{0}\right) g\left(x^{0}\right)^{-1}$. The arbitrariness in the choice of $g\left(x^{0}\right)$ is related to a $G L(6, C)$ gauge symmetry of the six-component formulation of the Proca fields. We will take $g\left(x^{0}\right)$ to be the identity matrix. This is a partial gauge-fixing because we do not fix $\gamma$. Changing $\gamma$ corresponds to a gauge transformation associated with a $G L(1, \mathrm{R})$ subgroup of $G L(6, \mathrm{C})$, namely, $\Psi_{\gamma} \rightarrow \Psi_{\gamma^{\prime}}=g\left(\gamma, \gamma^{\prime}\right) \Psi_{\gamma}$ where $g\left(\gamma, \gamma^{\prime}\right)$ $=(1 / 2 \gamma)\left(\begin{array}{c}\gamma+\gamma^{\prime} \gamma-\gamma^{\prime} \\ \gamma-\gamma^{\prime} \\ \gamma+\gamma^{\prime}\end{array}\right) \otimes \lambda_{0}$. This gauge symmetry has no physical significance, and as we shall see our final results will not depend on $\gamma$.)

The six-component vectors $\Psi\left(x^{0}\right)$ belong to $C^{2} \otimes \tilde{\mathcal{H}}$. If we endow the latter with the inner product $\langle\cdot, \cdot\rangle$ defined by

$$
\langle\xi, \zeta\rangle:=\sum_{i=1}^{2}\left\langle\xi^{i}, \zeta^{i}\right\rangle,
$$

for all $\xi=\left(\begin{array}{c}\xi^{1} \\ \xi^{1}\end{array}\right), \zeta=\left(\begin{array}{c}\zeta^{1} \\ \zeta^{2}\end{array}\right) \in \mathbb{C}^{2} \otimes \widetilde{\mathcal{H}}$, and denote the corresponding Hilbert space, namely, $\tilde{\mathcal{H}} \oplus \tilde{\mathcal{H}}$, by $\mathcal{H}^{\prime}$, we can view $\Psi^{\prime}\left(x^{0}\right)$ as elements of $\mathcal{H}^{\prime}$ and identify $H$ with a linear operator acting in $\mathcal{H}^{\prime}$. One can easily check that $H: \mathcal{H}^{\prime} \rightarrow \mathcal{H}^{\prime}$ is not a Hermitian operator, but it satisfies $H^{\dagger}=\Sigma_{3} H \Sigma_{3}^{-1}$, i.e., it is $\Sigma_{3}$-pseudo-Hermitian. This implies that $H$ is self-adjoint with respect to the inner product $\langle\langle\cdot, \cdot\rangle\rangle_{\Sigma_{3}}:=\left\langle\cdot, \Sigma_{3} \cdot\right\rangle$ on $\mathbb{C}^{2} \otimes \widetilde{\mathcal{H}}$. This in turn induces the following inner product on the space (14) of the Proca fields:

$$
\begin{aligned}
\left(\left(A, A^{\prime}\right)\right)_{\Sigma_{3}} & :=\frac{g}{2 \gamma}\left\langle\left\langle\Psi\left(x^{0}\right), \Psi^{\prime}\left(x^{0}\right)\right\rangle\right\rangle_{\Sigma_{3}}=\frac{g}{2 \gamma}\left\langle\Psi\left(x^{0}\right), \Sigma_{3} \Psi^{\prime}\left(x^{0}\right)\right\rangle \\
& =i g\left[\left\langle\mathbf{A},\left(\dot{\mathbf{A}}^{\prime}+\nabla A^{\prime 0}\right)>-\left\langle\left(\dot{\mathbf{A}}+\nabla A^{0}\right), \mathbf{A}^{\prime}>\right],\right.\right.
\end{aligned}
$$

where $g \in \mathbb{R}^{+}$is a constant. Because of $\Sigma_{3}$-pseudo-Hermiticity of $H$, this inner product, which is sometimes called the Proca inner product, is invariant under the time evolution generated by $H$, but it is obviously indefinite.

The dynamical invariance of (41) is associated with a conserved current density, namely, ${ }^{43,24}$ 


$$
J_{\Sigma_{3}}^{\mu}(x):=i g\left[A_{\nu}(x)^{*} F^{\nu \mu}(x)-F^{\nu \mu}(x)^{*} A_{\nu}(x)\right] .
$$

This is the spin-one analog of the KG current density. We can express the Proca inner product in a manifestly covariant form in terms of the current density (42). But because this inner product is indefinite, it cannot be used to make the solution space of the Proca equation into a genuine inner product space. Again in analogy to the case of KG fields, one may pursue the approach of indefinite-metric quantum theories ${ }^{18}$ and restrict to the subspace of positive-energy solutions (e.g., see Ref. 44). But this scheme has the same difficulties as the corresponding treatment of the KG fields. (For example, although the inner product (41) restricted to the positive-energy solutions is positive definite, $J_{\Sigma_{3}}^{0}(x)$ that should correspond to the probability density is not generally positive definite even for positive-energy fields.)

Next, we will use the positive definiteness of $D$ to show that $H$ is a diagonalizable operator with a real spectrum. This suggests that it is $\eta_{+}$-pseudo-Hermitian for a positive-definite metric operator $\eta_{+}{ }^{32}$ Therefore, $H$ is Hermitian with respect to the positive-definite inner product: $\langle\langle\cdot, \cdot\rangle\rangle_{\eta_{+}}:=\left\langle\cdot, \eta_{+} \cdot\right\rangle$. The construction of $\eta_{+}$requires the solution of the eigenvalue problem for $H$ and $H^{\dagger}$.

The eigenvalue problem for the Hamiltonian (36) may be easily solved. $H$ has a symmetry generated by

$$
\Lambda:=\sigma_{0} \otimes \mathfrak{h},
$$

which is the helicity operator in the six-component representation of the Proca fields. (Hereafter we will omit $\otimes$ wherever there is no risk of confusion.) The simultaneous eigenvectors of $H$ and $\Lambda$ are given by

$$
\begin{gathered}
\Psi_{\epsilon, h}(\mathbf{k}):=\frac{1}{2}\left(\begin{array}{c}
\left(r_{k}^{-1}+\epsilon r_{k}\right) \mathbf{u}_{\epsilon, h}(\mathbf{k})-\gamma r_{k}^{-1} \mathbf{k} u_{\epsilon, h}^{0}(\mathbf{k}) \\
\left(r_{k}^{-1}-\epsilon r_{k}\right) \mathbf{u}_{\epsilon, h}(\mathbf{k})+\gamma r_{k}^{-1} \mathbf{k} u_{\epsilon, h}^{0}(\mathbf{k})
\end{array}\right) \phi_{\mathbf{k}}, \\
H \Psi_{\epsilon, h}(\mathbf{k})=E_{\epsilon}(\mathbf{k}) \Psi_{\epsilon, h}(\mathbf{k}), \quad \Lambda \Psi_{\epsilon, h}(\mathbf{k})=h \Psi_{\epsilon, h}(\mathbf{k}),
\end{gathered}
$$

where $\epsilon \in\{-1,1\}, h \in\{-1,0,+1\}, \mathbf{k} \in \mathbb{R}^{3}, k:=|\mathbf{k}|$,

$$
\omega_{\mathbf{k}}:=\sqrt{k^{2}+\mathcal{M}^{2}}, \quad r_{k}:=\sqrt{\gamma \omega_{\mathbf{k}}}, \quad E_{\epsilon}(\mathbf{k}):=\epsilon \hbar \omega_{\mathbf{k}},
$$

$u_{\epsilon, h}(\mathbf{k})$ are circular polarization vectors $(23)$ and $\phi_{\mathbf{k}}$ are eigenvectors of $D$ corresponding to the eigenvalues $\omega_{\mathbf{k}}$. The eigenvectors $\Psi_{\epsilon, h}(\mathbf{k})$ together with

$$
\Phi_{\epsilon, h}(\mathbf{k}):=\frac{1}{2}\left(\begin{array}{c}
\left(r_{k}+\epsilon r_{k}^{-1}\right) \mathbf{u}_{\epsilon, h}(\mathbf{k})-\epsilon \gamma r_{k}^{-1} \mathbf{k} u_{\epsilon, h}^{0}(\mathbf{k}) \\
\left(r_{k}-\epsilon r_{k}^{-1}\right) \mathbf{u}_{\epsilon, h}(\mathbf{k})-\epsilon \gamma r_{k}^{-1} \mathbf{k} u_{\epsilon, h}^{0}(\mathbf{k})
\end{array}\right) \phi_{\mathbf{k}},
$$

form a complete biorthonormal system for the Hilbert space. This means that

$$
\left\langle\Psi_{\epsilon, h}(\mathbf{k}), \Phi_{\epsilon^{\prime}, h^{\prime}}\left(\mathbf{k}^{\prime}\right)\right\rangle=\delta_{\epsilon \in \epsilon^{\prime}} \delta_{h, h^{\prime}} \delta^{3}\left(\mathbf{k}-\mathbf{k}^{\prime}\right), \quad \sum_{\epsilon= \pm} \sum_{h=0, \pm 1} \int_{\mathbb{R}^{3}} d^{3} \mathbf{k}\left|\Psi_{\epsilon, h}(\mathbf{k})\right\rangle\left\langle\Phi_{\epsilon, h}(\mathbf{k})\right|=\Sigma_{0},
$$

where $\langle\cdot, \cdot\rangle$ stands for the inner product of $\mathcal{H}^{\prime}$, and for $\xi, \zeta \in \mathcal{H}^{\prime},|\xi\rangle\langle\zeta|$ is the operator defined by $|\xi\rangle\langle\zeta| \chi:=\langle\zeta, \chi\rangle \xi$, for all $\chi \in \mathcal{H}^{\prime}$. Similarly, one can check that indeed $\Phi_{\epsilon, h}(\mathbf{k})$ are simultaneous eigenvectors of $H^{\dagger}$ and $\Lambda^{\dagger}=\Lambda$ with the same eigenvalues, $H^{\dagger} \Phi_{\epsilon, h}(\mathbf{k})=E_{\epsilon}(\mathbf{k}) \Phi_{\epsilon, h}(\mathbf{k}), \Lambda \Phi_{\epsilon, h}(\mathbf{k})$ $={ }_{h} \Phi_{\epsilon, h}(\mathbf{k})$, and that $H$ and $\Lambda$ have the following spectral resolutions:

$$
H=\sum_{\epsilon= \pm} \sum_{h=0, \pm 1} \int_{\mathbb{R}^{3}} d^{3} \mathbf{k} E_{\epsilon}(\mathbf{k})\left|\Psi_{\epsilon, h}(\mathbf{k})\right\rangle\left\langle\Phi_{\epsilon, h}(\mathbf{k})\left|, \quad \Lambda=\sum_{\epsilon= \pm} \sum_{h=0, \pm 1} \int_{\mathbb{R}^{3}} d^{3} \mathbf{k} h\right| \Psi_{\epsilon, h}(\mathbf{k})\right\rangle\left\langle\Phi_{\epsilon, h}(\mathbf{k})\right| .
$$

Another remarkable property of the biorthonormal system $\left\{\Psi_{\epsilon, h}(\mathbf{k}), \Phi_{\epsilon, h}(\mathbf{k})\right\}$ is that 


$$
\left\langle\Psi_{\epsilon, h}(\mathbf{k}), \Psi_{\epsilon^{\prime}, h^{\prime}}\left(\mathbf{k}^{\prime}\right)\right\rangle=\left\langle\Psi_{\epsilon^{\prime}, h^{\prime}}\left(\mathbf{k}^{\prime}\right), \Psi_{\epsilon, h}(\mathbf{k})\right\rangle=\epsilon \epsilon^{\prime}\left\langle\Phi_{\epsilon, h}(\mathbf{k}), \Phi_{\epsilon^{\prime}, h^{\prime}}\left(\mathbf{k}^{\prime}\right)\right\rangle .
$$

Using the properties of the polarization vectors, i.e., Eqs. (19) and (22), we can compute the positive-definite metric operator, $\eta_{+}: \mathcal{H}^{\prime} \rightarrow \mathcal{H}^{\prime}$, associated with the biorthonormal system $\left\{\Psi_{\epsilon, h}(\mathbf{k}), \Phi_{\epsilon, h}(\mathbf{k})\right\}$. The result is

$$
\eta_{+}=\sum_{\epsilon= \pm} \sum_{h=0, \pm 1} \int_{\mathbb{R}^{3}} d^{3} \mathbf{k}\left|\Phi_{\epsilon, h}(\mathbf{k})\right\rangle\left\langle\Phi_{\epsilon, h}(\mathbf{k})\right|=\frac{D^{-1 / 2}}{2}\left(\begin{array}{ll}
H_{1} & H_{2} \\
H_{2} & H_{1}
\end{array}\right) .
$$

The inverse of $\eta_{+}$has the form

$$
\eta_{+}^{-1}=\sum_{\epsilon= \pm} \sum_{h=0, \pm 1} \int_{\mathbb{R}^{3}} d^{3} \mathbf{k}\left|\Psi_{\epsilon, h}(\mathbf{k})\right\rangle\left\langle\Psi_{\epsilon, h}(\mathbf{k})\right|=\frac{D^{-1 / 2}}{2}\left(\begin{array}{cc}
H_{1} & -H_{2} \\
-H_{2} & H_{1}
\end{array}\right) .
$$

Now we are in a position to compute $\langle\langle\cdot, \cdot\rangle\rangle_{\eta_{+}}$. For all $\xi, \zeta \in \mathcal{H}^{\prime}$, we let $\boldsymbol{\xi}^{i}, \boldsymbol{\zeta}^{i}, \boldsymbol{\xi}_{ \pm}, \boldsymbol{\zeta}_{ \pm} \in \tilde{\mathcal{H}}$ be defined by

$$
\left(\begin{array}{l}
\xi^{1} \\
\xi^{2}
\end{array}\right):=\xi, \quad\left(\begin{array}{l}
\zeta^{1} \\
\zeta^{2}
\end{array}\right):=\zeta, \quad \xi_{ \pm}:=\xi^{1} \pm \xi^{2}, \quad \zeta_{ \pm}:=\zeta^{1} \pm \zeta^{2} .
$$

Then in view of (49), (37), (38), and (40),

$$
\left.\langle\langle\xi, \zeta\rangle\rangle_{\eta_{+}}=\frac{1}{2}\left[\gamma<\boldsymbol{\xi}_{+}, D^{-1 / 2}\left[\mathcal{M}^{2}+(\mathfrak{K} \cdot \mathbf{S})^{2}\right] \boldsymbol{\zeta}_{+}\right\rangle+\frac{1}{\gamma \mathcal{M}^{2}}\left\langle\boldsymbol{\xi}_{-}, D^{-1 / 2}\left[D-(\mathfrak{K} \cdot \mathbf{S})^{2}\right] \boldsymbol{\zeta}_{-}\right\rangle\right] .
$$

If we view $\mathcal{H}^{\prime}$ as a complex vector space and endow it with the inner product (52), we obtain a new inner product space whose Cauchy completion yields a Hilbert space which we denote by $\mathcal{K}$.

Next, for all $x^{0} \in \mathbb{R}$ we define $U_{x^{0}}: \mathcal{V} \rightarrow \mathcal{H}^{\prime}$ according to

$$
U_{x^{0} A}:=\frac{1}{2} \sqrt{\frac{\kappa}{\gamma \mathcal{M}}} \Psi\left(x^{0}\right), \quad \forall A \in \mathcal{V},
$$

where $\kappa \in \mathbb{R}^{+}$is a fixed but arbitrary constant. We can use $U_{x^{0}}$ to endow the complex vector space $\mathcal{V}$ of Proca fields with the positive-definite inner product,

$$
\left(\left(A, A^{\prime}\right)\right):=\left\langle\left\langle U_{x^{0}} A, U_{x^{0}} A^{\prime}\right\rangle\right\rangle_{\eta_{+}}=\frac{\kappa}{4 \gamma \mathcal{M}}\left\langle\left\langle\Psi\left(x^{0}\right), \Psi^{\prime}\left(x^{0}\right)\right\rangle\right\rangle_{\eta_{+}} .
$$

Because $\eta_{+}$does not depend on $x^{0}$, the inner product $\langle\langle\cdot, \cdot\rangle\rangle_{\eta_{+}}$is invariant under the dynamics generated by $H^{33}$ This in turn implies that the right-hand side of (54) should be $x^{0}$-independent. In order to see this, we substitute (35), (52), and (54) and use (30) and (31) to derive

$$
\begin{aligned}
\left(\left(A, A^{\prime}\right)\right)= & \frac{\kappa}{2 \mathcal{M}}\left[<\mathbf{A}\left(x^{0}\right), D^{-1 / 2}\left[\mathcal{M}^{2}+(\mathfrak{K} \cdot \mathbf{S})^{2}\right] \mathbf{A}^{\prime}\left(x^{0}\right)>\right. \\
& \left.+\mathcal{M}^{-2}<\left(\dot{\mathbf{A}}\left(x^{0}\right)+i \mathfrak{K} A^{0}\left(x^{0}\right)\right), D^{-1 / 2}\left[D-(\mathfrak{K} \cdot \mathbf{S})^{2}\right]\left(\dot{\mathbf{A}}^{\prime}\left(x^{0}\right)+i \mathfrak{K} A^{\prime 0}\left(x^{0}\right)\right)>\right] \\
= & \frac{\kappa}{2 \mathcal{M}}\left[<\mathbf{A}\left(x^{0}\right), D^{-1 / 2} \dot{\mathbf{E}}^{\prime}\left(x^{0}\right)>-<\mathbf{E}\left(x^{0}\right), D^{-1 / 2} \dot{\mathbf{A}}^{\prime}\left(x^{0}\right)>\right],
\end{aligned}
$$

where $\mathbf{E}\left(x^{0}\right)=-\dot{\mathbf{A}}\left(x^{0}\right)-i \mathfrak{K} A^{0}\left(x^{0}\right)$. We can use (11) or equivalently (30) to check that the $x^{0}$-derivative of the right-hand side of (55) vanishes identically. Therefore, (55) provides a welldefined inner product on $\mathcal{V}$. Endowing $\mathcal{V}$ with this inner product and (Cauchy) completing the 
resulting inner product space, we obtain a separable Hilbert space, which we shall denote by $\mathcal{H}$. This is the physical Hilbert space of the RQM of the Proca fields.

The inner product (55) is identical with an inner product obtained in Ref. 29. We will show in Sec. IV that it is a special example of a larger class of invariant inner products and that it has the following appealing properties.

(1) It is not only positive-definite but relativistically invariant. (We give a manifestly covariant expression for this inner product in Sec. V.)

(2) Its restriction to the subspace of positive-frequency Proca fields coincides with the restriction of the indefinite Proca inner product (41) to this subspace.

As seen from (54), the operator $U_{x^{0}}$ for any value of $x^{0} \in \mathrm{R}$ is a unitary operator mapping $\mathcal{H}$ to $\mathcal{K}$. Following Refs. 26 and 27 we can use this unitary operator to define a Hamiltonian operator $\mathrm{h}$ acting in $\mathcal{H}$ that is unitary equivalent to $H$. Let $x_{0}^{0} \in \mathbb{R}$ be an arbitrary initial $x^{0}$, and $\mathrm{h}: \mathcal{H} \rightarrow \mathcal{H}$ be defined by

$$
\mathrm{h}:=U_{x_{0}^{0}}^{-1} H U_{x_{0}^{0}} .
$$

Then, using (29), (35)-(38), and (53), we can easily show that for any $A \in \mathcal{V}$,

$$
\mathrm{h} A=i \hbar \dot{A},
$$

where $\dot{A}$ is the element of $\mathcal{V}$ defined by: $\dot{A}\left(x^{0}\right):=\left(d / d x^{0}\right) A\left(x^{0}\right)$ for all $x^{0} \in \mathbb{R}$. As discussed in Ref. 26 for the case of KG fields, one must not confuse (57) with a time-dependent Schrödinger equation giving the $x^{0}$-dependence of $A\left(x^{0}\right)$. This equation actually defines the action of the operator $\mathrm{h}$ on the field $A$. h generates a time evolution, through the Schrödinger equation

$$
i \hbar \frac{d}{d x^{0}} A_{x^{0}}=\mathrm{h} A_{x^{0}},
$$

that coincides with a time-translation in the space $\mathcal{V}$ of the Proca fields; if $A_{0}=A_{x_{0}^{0}}$ is the initial value for the one-parameter family of elements $A_{x^{0}}$ of $\mathcal{V}$, then for all $x^{0}, x^{0^{\prime}} \in \mathbb{R}, A_{x^{0}}\left(x^{0^{\prime}}\right)$ $=\left(e^{-i\left(x^{0}-x_{0}^{0}\right) \mathrm{h} / \hbar} A_{0}\right)\left(x^{0^{\prime}}\right)=A_{0}\left(x^{0^{\prime}}+x^{0}-x_{0}^{0}\right)$. Furthermore, using the fact that $U_{x_{0}^{0}}$ is a unitary operator and that $H$ is Hermitian with respect to the inner product (52) of $\mathcal{K}$, we can infer that $\mathrm{h}$ is Hermitian with respect to the inner product $(54)$ of $\mathcal{H}$. This shows that time translations correspond to unitary transformations of the physical Hilbert space $\mathcal{H}$.

Next, we define $\mathcal{U}: \mathcal{H} \rightarrow \mathcal{H}^{\prime}$ and $H^{\prime}: \mathcal{H}^{\prime} \rightarrow \mathcal{H}^{\prime}$ by

$$
\begin{gathered}
\mathcal{U}:=\rho U_{x_{0}^{0}}, \\
H^{\prime}:=\mathcal{U} \mathrm{h} \mathcal{U}^{-1}=\rho H \rho^{-1},
\end{gathered}
$$

where $\rho$ is the unique positive square root of $\eta_{+}$, i.e., $\rho:=\sqrt{\eta_{+}}$. It is not difficult to see that

$$
\rho=\frac{1}{2 \mathcal{M} \sqrt{\gamma}}\left(\begin{array}{ll}
\rho_{+} & \rho_{-} \\
\rho_{-} & \rho_{+}
\end{array}\right), \quad \rho^{-1}=\frac{1}{2 \mathcal{M} \sqrt{\gamma}}\left(\begin{array}{cc}
\rho_{+} & -\rho_{-} \\
-\rho_{-} & \rho_{+}
\end{array}\right),
$$

where $\rho_{ \pm}: \widetilde{\mathcal{H}} \rightarrow \tilde{\mathcal{H}}$ are Hermitian operators given by

$$
\rho_{ \pm}:=(\gamma \mathcal{M} \mp 1)\left[D^{1 / 4}-\mathcal{M} D^{-1 / 4}\right] \mathfrak{h}^{2}+\gamma \mathcal{M}^{2} D^{-1 / 4} \pm D^{1 / 4},
$$

and $\mathfrak{h}$ is the helicity operator (24). We can check that the operator $\rho$ viewed as mapping $\mathcal{K}$ to $\mathcal{H}^{\prime}$ is a unitary transformation; using $\rho^{\dagger}=\rho=\sqrt{\eta_{+}}$, we have $\langle\rho \xi, \rho \zeta\rangle=\left\langle\langle\xi, \zeta\rangle \eta_{\eta_{+}}\right.$for all $\xi, \zeta \in \mathcal{K}$. This in turn implies that $\mathcal{U}: \mathcal{H} \rightarrow \mathcal{H}^{\prime}$ is also a unitary transformation, 


$$
\left\langle\mathcal{U} A, \mathcal{U} A^{\prime}\right\rangle=\left(\left(A, A^{\prime}\right)\right), \quad \forall A, A^{\prime} \in \mathcal{H},
$$

and that $H^{\prime}$ must be a Hermitian Hamiltonian operator acting in $\mathcal{H}^{\prime}$.

We can compute $H^{\prime}$ by substituting (61), (62), and (36)-(38) in (60). This yields

$$
H^{\prime}=\hbar\left(\begin{array}{cc}
\sqrt{D} & 0 \\
0 & -\sqrt{D}
\end{array}\right)=\hbar \sqrt{D} \Sigma_{3},
$$

which is manifestly Hermitian with respect to the inner product $\langle\cdot, \cdot\rangle$ of $\mathcal{H}^{\prime}$. The Hamiltonian $H^{\prime}$ is precisely the Foldy Hamiltonian. ${ }^{17,16}$ Here we obtained it by a systematic application of the methods of PHQM. ${ }^{5}$

Next, we derive the explicit form of $\mathcal{U}$ and its inverse. Using (59), (61), (62), (53), and (35), we have for all $A \in \mathcal{H}$

$$
\mathcal{U} A=\frac{1}{2} \sqrt{\frac{\kappa}{\mathcal{M}}}\left(\begin{array}{l}
\mathfrak{U} \mathbf{A}\left(x_{0}^{0}\right)-i \mathfrak{U}^{-1} \mathbf{E}\left(x_{0}^{0}\right) \\
\mathfrak{U} \mathbf{A}\left(x_{0}^{0}\right)+i \mathfrak{U}^{-1} \mathbf{E}\left(x_{0}^{0}\right)
\end{array}\right),
$$

where the operator $\mathfrak{U}: \widetilde{\mathcal{H}} \rightarrow \tilde{\mathcal{H}}$ and its inverse are given by

$$
\mathfrak{U}=\left[D^{1 / 4}-\mathcal{M} D^{-1 / 4}\right] \mathfrak{h}^{2}+\mathcal{M} D^{-1 / 4}, \quad \mathfrak{U}^{-1}=\left[D^{-1 / 4}-\mathcal{M}^{-1} D^{1 / 4}\right] \mathfrak{h}^{2}+\mathcal{M}^{-1} D^{1 / 4}
$$

(Transformation (65) is known as the Foldy's transformation, although it differs slightly from the expression given by Foldy ${ }^{17}$ and Case, ${ }^{16}$ namely, $\mathcal{U}_{\text {Foldy }}:=\Sigma_{3} \mathcal{U}$. This difference turns out not to have any effect on the definition of the physical observables such as the position operator. It is interesting to see that the arbitrary parameter $\gamma$, introduced in the six-component formulation of the Proca equation, does not appear in (64) and (65).) The inverse $\mathcal{U}^{-1}$ of $\mathcal{U}$ is also easy to calculate. Let $\xi \in \mathcal{H}^{\prime}$ be a six-component vector [as in (51)] with components $\xi^{1}$ and $\xi^{2}$. Then in view of (65), $\mathcal{U}^{-1} \xi$ is the Proca field $A \in \mathcal{H}$ satisfying the following initial conditions:

$$
\begin{gathered}
A^{0}\left(x_{0}^{0}\right)=\frac{D^{-1 / 4}}{\sqrt{\mathcal{M} \kappa}} \mathfrak{K} \cdot\left(\xi^{1}-\xi^{2}\right), \quad \dot{A}^{0}\left(x_{0}^{0}\right)=-i \frac{D^{1 / 4}}{\sqrt{\mathcal{M} \kappa}} \mathfrak{K} \cdot\left(\xi^{1}+\xi^{2}\right), \\
\mathbf{A}\left(x_{0}^{0}\right)=\sqrt{\frac{\mathcal{M}}{\kappa}} \mathfrak{U}^{-1}\left(\xi^{1}+\xi^{2}\right), \quad \dot{\mathbf{A}}\left(x_{0}^{0}\right)=-i \sqrt{\frac{\mathcal{M}}{\kappa}} D^{1 / 2} \mathfrak{U}^{-1}\left(\xi^{1}-\xi^{2}\right),
\end{gathered}
$$

where we have made use of Eqs. (15), (30), (33), (35), (53), (61), and (62). By virtue of (17), for all $x^{0} \in \mathbb{R}$, we have

$$
\begin{gathered}
A^{0}\left(x^{0}\right)=\frac{D^{-1 / 4}}{\sqrt{\mathcal{M} \kappa}}\left[e^{-i\left(x^{0}-x_{0}^{0}\right) D^{1 / 2}} \mathfrak{K} \cdot \boldsymbol{\xi}^{1}-e^{i\left(x^{0}-x_{0}^{0}\right) D^{1 / 2}} \mathfrak{K} \cdot \boldsymbol{\xi}^{2}\right], \\
\mathbf{A}\left(x^{0}\right)=\sqrt{\frac{\mathcal{M}}{\kappa}} \mathfrak{U}^{-1}\left[e^{-i\left(x^{0}-x_{0}^{0}\right) D^{1 / 2}} \xi^{1}+e^{i\left(x^{0}-x_{0}^{0}\right) D^{1 / 2}} \xi^{2}\right] .
\end{gathered}
$$

In complete analogy with the case of $\mathrm{KG}$ fields, ${ }^{27}$ we find that the pairs $(\mathcal{H}, \mathrm{h}),(\mathcal{K}, H)$, and $\left(\mathcal{H}^{\prime}, H^{\prime}\right)$ are unitarily equivalent. Therefore they represent the same quantum system.

\section{III. $\mathcal{P} \mathcal{T}, \mathcal{C}$, AND $\mathcal{C} \mathcal{P} \mathcal{T}$-SYMMETRIES OF PROCA FIELDS}

According to Ref. 35, the generalized parity $(\mathcal{P})$, time-reversal $(\mathcal{T})$, and charge grading or chirality $(\mathcal{C})$ operators for the six-component Proca fields are given by

$$
\mathcal{P}:=\sum_{\epsilon= \pm} \sum_{\sigma=1}^{3} \int_{\mathbb{R}^{3}} d^{3} \mathbf{k} \sigma_{\epsilon, \sigma}(\mathbf{k})\left|\Phi_{\epsilon, \sigma}(\mathbf{k})\right\rangle\left\langle\Phi_{\epsilon, \sigma}(\mathbf{k})\right|,
$$




$$
\begin{gathered}
\mathcal{T}:=\sum_{\epsilon= \pm} \sum_{\sigma=1}^{3} \int_{\mathbb{R}^{3}} d^{3} \mathbf{k} \sigma_{\epsilon, \sigma}(\mathbf{k})\left|\Phi_{\epsilon, \sigma}(\mathbf{k})\right\rangle \star\left\langle\Phi_{\epsilon, \sigma}(\mathbf{k})\right|, \\
\mathcal{C}:=\sum_{\epsilon= \pm} \sum_{\sigma=1}^{3} \int_{\mathbb{R}^{3}} d^{3} \mathbf{k} \sigma_{\epsilon, \sigma}(\mathbf{k})\left|\Psi_{\epsilon, \sigma}(\mathbf{k})\right\rangle\left\langle\Phi_{\epsilon, \sigma}(\mathbf{k})\right|,
\end{gathered}
$$

where $\sigma_{\varepsilon, \sigma}(\mathbf{k})$ are arbitrary signs $( \pm)$ and $\star$ is the complex-conjugation operator defined for all complex numbers $z$ and state vectors $\Psi, \Phi$, by $\star z:=z^{*}$ and $(\star\langle\Phi|)|\Psi\rangle:=\star\langle\Phi \mid \Psi\rangle=\langle\Psi \mid \Phi\rangle$. Note that we use the biorthonormal basis $\left\{\Psi_{\varepsilon, \sigma}(\mathbf{k}), \Phi_{\varepsilon, \sigma}(\mathbf{k})\right\}$ to define $\mathcal{P}, \mathcal{T}$, and $\mathcal{C}$. This basis can be obtained by replacing the circular polarization vectors $u_{\varepsilon, h}(\mathbf{k})$ by the linear polarization vectors $a_{\varepsilon}(\mathbf{k}, \sigma)$ in (44) and (46).

Clearly there is an infinity of choices for the signs $\sigma_{\varepsilon, \sigma}(\mathbf{k})$ each leading to a different $\mathcal{P}, \mathcal{T}$, and $\mathcal{C}$. Following Ref. 27 we will adopt the natural choice associated with the label $\varepsilon$ appearing in the expression for the eigenvalues and eigenvectors of $H$. This yields

$$
\begin{aligned}
\mathcal{P} & :=\sum_{\epsilon= \pm} \sum_{h=0, \pm 1} \int_{\mathbb{R}^{3}} d^{3} \mathbf{k} \epsilon\left|\Phi_{\epsilon, h}(\mathbf{k})\right\rangle\left\langle\Phi_{\epsilon, h}(\mathbf{k})\right|, \\
\mathcal{T} & :=\sum_{\epsilon= \pm} \sum_{h=0, \pm 1} \int_{\mathbb{R}^{3}} d^{3} \mathbf{k} \epsilon\left|\Phi_{\epsilon, h}(\mathbf{k})\right\rangle \star\left\langle\Phi_{\epsilon,-h}(\mathbf{k})\right|, \\
\mathcal{C} & :=\sum_{\epsilon= \pm} \sum_{h=0, \pm 1} \int_{\mathbb{R}^{3}} d^{3} \mathbf{k} \epsilon\left|\Psi_{\epsilon, h}(\mathbf{k})\right\rangle\left\langle\Phi_{\epsilon, h}(\mathbf{k})\right| .
\end{aligned}
$$

The consequences of these relations are identical with those obtained in Ref. 27 for the KG fields. Because of the close analogy with the case of KG fields, here we omit the details and give a summary of the relevant results.

Substituting (44) and (46) in (74)-(76) yields

$$
\mathcal{P}=\Sigma_{3}, \quad \mathcal{T}=\Sigma_{3} \star, \quad \mathcal{P} \mathcal{T}=\star .
$$

Hence, the $\mathcal{P} \mathcal{T}$-symmetry of the Hamiltonian (36) means that it is a real operator. Note that $\mathcal{P}^{2}$ $=\mathcal{T}^{2}=\Sigma_{0}$ and $\mathcal{P} \mathcal{T}=\mathcal{P} \cdot \mathcal{T}$, which is a direct consequence of $(48) .{ }^{35}$ Similarly, we find

$$
\mathcal{C}=\frac{D^{-1 / 2}}{2}\left(\begin{array}{cc}
H_{1} & H_{2} \\
-H_{2} & -H_{1}
\end{array}\right)=\hbar^{-1} D^{-1 / 2} H=\frac{H}{\sqrt{H^{2}}},
$$

which in view of (49) is consistent with the identity $\mathcal{C}=\eta_{+}^{-1} \mathcal{P}$ (equivalently $\eta_{+}=\mathcal{P C}$ (Note that $\mathcal{P}$, $\mathcal{T}$, and the metric operator $\eta_{+}$depend on the choice of the biorthonormal system, while $\mathcal{C}$ is independent. In Ref. 45 this fact has not been considered in the factorization of the metric operator.)). ${ }^{35}$ According to (78), $\mathcal{C}$ is a $\mathbb{Z}_{2}$-grading operator for the Hilbert space that splits it into the spans of the eigenvectors of $H$ with positive and negative eigenvalues, respectively.

We can use the unitary operator $\rho: \mathcal{K} \rightarrow \mathcal{H}^{\prime}$ to express the generalized parity, time-reversal, and chirality operators in the Foldy representation, namely, $\mathcal{P}^{\prime}:=\rho \mathcal{P} \rho^{-1}, \mathcal{T}^{\prime}:=\rho \mathcal{T} \rho^{-1}$, and $\mathcal{C}^{\prime}$ $:=\rho \mathcal{C} \rho^{-1}$. The symmetry generators $\mathcal{P}^{\prime} \mathcal{T}^{\prime}$ and $\mathcal{C}^{\prime}$ that commute with the Foldy Hamiltonian $H^{\prime}$ have the form

$$
\mathcal{P}^{\prime} \mathcal{T}^{\prime}=\mathcal{P} \mathcal{T}=\star, \quad \mathcal{C}^{\prime}=\frac{H^{\prime}}{\sqrt{H^{\prime 2}}}=\Sigma_{3} .
$$

Clearly, the $\mathcal{P}^{\prime} \mathcal{T}^{\prime}$-symmetry of $H^{\prime}$ is related to the fact that $H^{\prime}$ is a real operator, and the $\mathcal{C}^{\prime}$-symmetry of $H^{\prime}$ arises because it is proportional to $\mathcal{C}^{\prime}$. 
Similarly we can use the unitary operator $\mathcal{U}: \mathcal{H} \rightarrow \mathcal{H}^{\prime}$ to define the generalized parity, timereversal, and chirality operators for the ordinary Proca fields $A \in \mathcal{H}$. This yields

$$
\mathrm{P}:=\mathcal{U}^{-1} \mathcal{P}^{\prime} \mathcal{U}, \quad \mathrm{T}:=\mathcal{U}^{-1} \mathcal{T}^{\prime} \mathcal{U}, \quad \mathrm{c}:=\mathcal{U}^{-1} \mathcal{C}^{\prime} \mathcal{U}
$$

Using Eqs. (65)-(68) and (17), we may obtain explicit expressions for P and T. Here we give the corresponding expressions for PT and C, that actually generate symmetries of the Hamiltonian $\mathrm{h}$. The result is, for all $A \in \mathcal{H}$ and $x^{0} \in \mathbb{R}$,

$$
\begin{gathered}
{[\mathrm{PT} A]\left(x^{0}\right)=\left(-A^{0}\left(-x^{0}\right)^{*}, \mathbf{A}\left(-x^{0}\right)^{*}\right),} \\
{[\mathrm{C} A]\left(x^{0}\right)=i D^{-1 / 2} \dot{A}\left(x^{0}\right)=: A_{c}\left(x^{0}\right) .}
\end{gathered}
$$

These relations imply that, similarly to the case of KG field, ${ }^{27} \mathrm{PT}$ is just the ordinary time-reversal operator, ${ }^{17}$ and "the PT-symmetry of $\mathrm{h}$ means that the order in which one performs a time translation and a time-reversal transformation on a Proca field is not important." Furthermore, chirality operator acting in $\mathcal{H}$ is simply given by $\mathrm{C}=i D^{-1 / 2} \partial_{0}$, which is a Lorentz scalar. ${ }^{6}$ Recalling $\mathrm{C}^{2} A=A$, we observe that $\mathrm{C}: \mathcal{V} \rightarrow \mathcal{V}$ is an involution, $\mathrm{C}^{2}=1$. Therefore, we can use it to split $\mathcal{V}$ into the subspaces $\mathcal{V}_{ \pm}$of \pm -energy Proca fields according to $\mathcal{V}_{ \pm}:=\left\{A_{ \pm} \in \mathcal{V} \mid \mathrm{C} A_{ \pm}= \pm A_{ \pm}\right\}$. Clearly, for any $A \in \mathcal{V}$, we can use $\mathrm{C}$ to introduce the corresponding \pm -energy components,

$$
A_{ \pm}:=\frac{1}{2}(A \pm \mathrm{C} A) \in \mathcal{V}_{ \pm} .
$$

Clearly $A=A_{+}+A_{-}$, so $\mathcal{V}=\mathcal{V}_{+} \oplus \mathcal{V}_{-}$. Restricting the inner product (55) to $\mathcal{V}_{ \pm}$(and Cauchy completing the resulting inner product spaces) we obtain Hilbert subspaces $\mathcal{H}_{ \pm}$of $\mathcal{H}$.

Next we recall that $\mathcal{C}^{\prime}$ is a Hermitian operator acting in $\mathcal{H}^{\prime}$. Thus, in view of (80) and (63), we have $\left(\left(A, C A^{\prime}\right)\right)=\left(\left(\mathrm{C} A, A^{\prime}\right)\right)$. Therefore, $\mathrm{C}: \mathcal{H} \rightarrow \mathcal{H}$ is a Hermitian involution (This marks its similarity to the chirality operator $\gamma^{5}$ for spin $1 / 2$ fields. $)$ and for all $A_{ \pm} \in \mathcal{V}_{ \pm},\left(\left(A_{+}, A_{-}\right)\right)=0$. This in turns implies $\mathcal{H}=\mathcal{H}_{+} \oplus \mathcal{H}_{-}$. The generalized chirality operator $\mathrm{C}$ is actually the grading operator associated with this orthogonal direct sum decomposition of $\mathcal{H}$. In other words, similarly to the case of $\mathrm{KG}$ fields, ${ }^{27} \mathrm{C}$ is a Hermitian involution that decomposes the Hilbert space into its \pm -energy subspaces. As a result, "the $\mathrm{C}$-symmetry of $\mathrm{h}$ means that the energy of a free Proca field does not change sign under time translations."

\section{THE MOST GENERAL ADMISSIBLE INNER PRODUCT}

The metric operator $\eta_{+}$and the corresponding invariant positive-definite inner product depend on the choice of the biorthonormal system $\left\{\Psi_{\varepsilon, h}(\mathbf{k}), \Phi_{\varepsilon, h}(\mathbf{k})\right\}$. The most general invariant positivedefinite inner product corresponds, therefore, to the most general biorthonormal system that consists of the eigenvectors of $H$ and $H^{\dagger}$. In the construction of $\left\{\Psi_{\varepsilon, h}(\mathbf{k}), \Phi_{\varepsilon, h}(\mathbf{k})\right\}$, we have chosen a set of eigenvectors of $H$ that also diagonalize the helicity operator $\Lambda$. As a result, both $H$ and $\Lambda$ are $\eta_{+}$-pseudo-Hermitian. This in turn is equivalent to the condition that $H$ and $\Lambda$ are among the physical observables of the system. In the following we construct the most general Lorentz invariant positive-definite inner product that fulfills this condition. (This amounts to restricting the choice of the metric operator) in the spirit of Ref. 34. It does not, however, fix the metric operator (up to a trivial scaling) because $H$ and $\Lambda$ do not form an irreducible set of operators.)

The most general positive-definite operator $\widetilde{\eta}_{+}$that renders both $H$ and $\Lambda$ $\tilde{\eta}_{+}$-pseudo-Hermitian has the form $\tilde{\eta}_{+}:=\mathcal{A}^{\dagger} \eta_{+} \mathcal{A}$, where $\mathcal{A}$ is an invertible linear operator commuting with $H$ and $\Lambda .{ }^{39,35}$ We can express $\mathcal{A}$ as 


$$
\mathcal{A}=\sum_{\epsilon= \pm} \sum_{h=0, \pm 1} \int_{\mathbb{R}^{3}} d^{3} \mathbf{k} \alpha_{\epsilon, h}(\mathbf{k})\left|\Psi_{\epsilon, h}(\mathbf{k})\right\rangle\left\langle\Phi_{\epsilon, h}(\mathbf{k})\right|,
$$

where $\alpha_{\epsilon, h}(\mathbf{k})$ are arbitrary nonzero complex numbers. (The condition that $H$ is $\tilde{\eta}_{+}$-pseudo-Hermitian yields $\mathcal{A}=\Sigma_{\epsilon= \pm} \Sigma_{h=0, \pm 1} \Sigma_{h^{\prime}=0, \pm 1} \int_{\mathrm{R}^{3}} d^{3} \mathbf{k} \alpha_{h, h^{\prime}}^{\epsilon}(\mathbf{k})\left|\Psi_{\epsilon, h}(\mathbf{k})\right\rangle\left\langle\Phi_{\epsilon, h^{\prime}}(\mathbf{k})\right|$ which does not commute with $\Lambda$ unless $\alpha_{h, h^{\prime}}^{\epsilon}=\delta_{h, h^{\prime}} \alpha_{\epsilon, h}$. Equation (84) implies

$$
\widetilde{\eta}_{+}:=\mathcal{A}^{\dagger} \eta_{+} \mathcal{A}=\sum_{\epsilon= \pm} \sum_{h=0, \pm 1} \int_{\mathbb{R}^{3}} d^{3} \mathbf{k}\left|\alpha_{\epsilon, h}(\mathbf{k})\right|^{2}\left|\Phi_{\epsilon, h}(\mathbf{k})\right\rangle\left\langle\Phi_{\epsilon, h}(\mathbf{k})\right| .
$$

Substituting (44) and (46) in (85), and carrying out the necessary calculations, we find

$$
\tilde{\eta}_{+}=\left[L_{+}^{+} M+\left(L_{-}^{+}-L_{-}^{0}\right) \sigma_{3}-L_{+}^{0} N\right] \mathfrak{h}^{2}+\left[L_{+}^{-} M+L_{-}^{-} \sigma_{3}\right] \mathfrak{h}+L_{+}^{0} N+L_{-}^{0} \sigma_{3},
$$

where

$$
\begin{gathered}
L_{ \pm}^{\epsilon}:=\frac{1}{4} \int d^{3} \mathbf{k}\left[\left|\alpha_{+, 1}(\mathbf{k})\right|^{2}+\epsilon\left|\alpha_{+,-1}(\mathbf{k})\right|^{2} \pm\left|\alpha_{-, 1}(\mathbf{k})\right|^{2} \pm \epsilon\left|\alpha_{-,-1}(\mathbf{k})\right|^{2}\right]|\mathbf{k}\rangle\langle\mathbf{k}| \\
L_{ \pm}^{0}:=\frac{1}{2} \int d^{3} \mathbf{k}\left[\left|\alpha_{+, 0}(\mathbf{k})\right|^{2} \pm\left|\alpha_{-, 0}(\mathbf{k})\right|^{2}\right]|\mathbf{k}\rangle\langle\mathbf{k}| \\
M:=\frac{D^{-1 / 2}}{2 \gamma}\left(\begin{array}{cc}
\gamma^{2} D+1 & \gamma^{2} D-1 \\
\gamma^{2} D-1 & \gamma^{2} D+1
\end{array}\right) \\
N:=\frac{D^{-1 / 2}}{2 \gamma \mathcal{M}^{2}}\left(\begin{array}{ll}
\gamma^{2} \mathcal{M}^{4}+D & \gamma^{2} \mathcal{M}^{4}-D \\
\gamma^{2} \mathcal{M}^{4}-D & \gamma^{2} \mathcal{M}^{4}+D
\end{array}\right)
\end{gathered}
$$

Having computed $\widetilde{\eta}_{+}$we can easily obtain the corresponding inner product according to $\langle\langle\cdot, \cdot\rangle\rangle_{\tilde{\eta}_{+}}:=\left\langle\cdot, \tilde{\eta}_{+} \cdot\right\rangle$. This yields, for all $\xi, \zeta \in \mathcal{H}^{\prime}$,

$$
\left.\langle\langle\xi, \zeta\rangle\rangle_{\tilde{\eta}_{+}}=\frac{1}{2}\left[\gamma<\xi_{+}, \Theta_{+,+1} \zeta_{+}\right\rangle+\gamma^{-1}<\xi_{-}, \Theta_{+,-1} \zeta_{-}>\right]+\left\langle\xi^{1}, \Theta_{-, 0} \zeta^{1}>-<\xi^{2}, \Theta_{-, 0} \zeta^{2}\right\rangle
$$

where the operators $\Theta_{\epsilon, h}: \widetilde{\mathcal{H}} \rightarrow \widetilde{\mathcal{H}}$ are defined by

$$
\Theta_{\epsilon, h}:=\left[L_{\epsilon}^{+} D^{h / 2}-\mathcal{M}^{2 h} L_{\epsilon}^{0} D^{-h / 2}\right] \mathfrak{h}^{2}+L_{\epsilon}^{-} D^{h / 2} \mathfrak{h}+\mathcal{M}^{2 h} L_{\epsilon}^{0} D^{-h / 2} .
$$

If we view $\mathcal{H}^{\prime}$ as a complex vector space and endow it with this inner product, we obtain a new inner product space whose Cauchy completion yields a Hilbert space which we denote by $\widetilde{\mathcal{K}}$. Finally, we recall that, defining $\left(\left(A, A^{\prime}\right)\right)_{\{\mathfrak{a}\}}:=\left\langle\left\langle U_{x^{0}} A, U_{x^{0}} A^{\prime}\right\rangle\right\rangle_{\tilde{\eta}_{+}}$, we can endow the space $\mathcal{V}$ of the solutions of Proca equation with the positive-definite inner product,

$$
\begin{aligned}
\left(\left(A, A^{\prime}\right)\right)_{\{a\}}:= & \frac{\kappa}{2 \mathcal{M}}\left[<\mathbf{A}\left(x^{0}\right), \Theta_{+,+1} \mathbf{A}^{\prime}\left(x^{0}\right)>+<\mathbf{E}\left(x^{0}\right), \Theta_{+,-1} \mathbf{E}^{\prime}\left(x^{0}\right)>\right. \\
& \left.+i\left\{<\mathbf{E}\left(x^{0}\right), \Theta_{-, 0} \mathbf{A}^{\prime}\left(x^{0}\right)>-<\mathbf{A}\left(x^{0}\right), \Theta_{-, 0} \mathbf{E}^{\prime}\left(x^{0}\right)>\right\}\right],
\end{aligned}
$$

which is dynamically invariant. This fact may be checked directly by differentiating the right-hand side of (93) with respect to $x^{0}$ and making use of (11) or equivalently (30). 
By construction, (93) gives the most general positive-definite inner product that renders the generator of time translations as well as the helicity operator Hermitian. We next impose the condition that (93) be Lorentz invariant as well. This will give rise to the most general physically admissible inner product on the space of Proca fields.

To quantify the requirement that the right-hand side of (93) be a Lorentz scalar, we compute the inner product of two plane-wave solutions of the Proca equation, i.e., (27). Using (24), (32), (87), (88), and (92) in (93), we find after a rather lengthy calculation,

$$
\left(\left(A_{\epsilon, h}(\mathbf{k}), A_{\epsilon^{\prime}, h^{\prime}}\left(\mathbf{k}^{\prime}\right)\right)\right)_{\{\mathfrak{a}\}}=\frac{\kappa}{\mathcal{M}}\left|N_{\epsilon, h}(\mathbf{k})\right|^{2} \mathfrak{a}_{\epsilon, h} \omega_{\mathbf{k}} \delta^{3}\left(\mathbf{k}-\mathbf{k}^{\prime}\right) \delta_{\epsilon, \epsilon^{\prime}} \delta_{h, h^{\prime}},
$$

where we have introduced the abbreviation $\mathfrak{a}_{\epsilon, h}:=\left|\alpha_{\epsilon, h}\right|^{2}$. Because $\mathfrak{a}_{\epsilon, h}$ are positive real numbers and the right-hand side of (94) does not involve $x^{0}$, this equation provides an explicit demonstration of the invariance and positive definiteness of the inner product (93).

Next, we recall from Refs. 3 and 46 that relativistically invariant normalization of two planewave solutions is given by $\left(\left(A_{\epsilon, h}(\mathbf{k} ; x), A_{\epsilon^{\prime}, h^{\prime}}\left(\mathbf{k}^{\prime} ; x\right)\right)\right)=2 \omega_{\mathbf{k}} \delta^{3}\left(\mathbf{k}-\mathbf{k}^{\prime}\right) \delta_{\epsilon, \epsilon^{\prime}} \delta_{h, h^{\prime}}$. This together with the fact that $N_{\epsilon, h}(\mathbf{k})$ must be a Lorentz scalar so that $A_{\epsilon, h}(\mathbf{k} ; x)$ is a four-vector shows that $\mathfrak{a}_{\epsilon, h}$ are dimensionless positive real numbers, i.e., they do not depend on $\mathbf{k}$ and obey the same Lorentz transformation rule as scalars. This result ensures the relativistic invariance of the inner product of any two solutions $A$ and $A^{\prime}$ having the general form:

$$
A=\sum_{\epsilon= \pm} \sum_{h=0, \pm 1} \int_{\mathbb{R}^{3}} d^{3} \mathbf{k} c_{\epsilon, h}(\mathbf{k}) A_{\epsilon, h}(\mathbf{k}),
$$

where $c_{\epsilon, h}(\mathbf{k})$ are complex coefficients. In view of (94) and (95) and the fact that the inner product (93) is a Hermitian sesquilinear form, we obtain

$$
\left(\left(A, A^{\prime}\right)\right)_{\{\mathfrak{a}\}}=\frac{\kappa}{\mathcal{M}} \sum_{\epsilon= \pm} \sum_{h=0, \pm 1} \int_{\mathbb{R}^{3}} d^{3} \mathbf{k} \mathfrak{a}_{\epsilon, h} \omega_{\mathbf{k}}\left|N_{\epsilon, h}(\mathbf{k})\right|^{2} c_{\epsilon, h}^{*}(\mathbf{k}) c_{\epsilon, h}^{\prime}(\mathbf{k}) .
$$

Because $A$ and $A_{\epsilon, h}(\mathbf{k})$ are four-vectors and $d^{3} \mathbf{k} / \omega_{\mathbf{k}}$ is a relativistically invariant measure, ${ }^{3,46}$ $c_{\epsilon, h}(\mathbf{k})$ obeys the same Lorentz transformation rule as $\omega_{\mathbf{k}}^{-1}$. This in turn implies that in order for the inner product (96) to be scalars, $\mathfrak{a}_{\epsilon, h}$ must transform as scalars. Therefore, in view of (87) and (88), we conclude that $L_{ \pm}^{ \pm}$and $L_{ \pm}^{0}$ are dimensionless real numbers given by

$$
L_{ \pm}^{\epsilon}=\frac{1}{4}\left[\mathfrak{a}_{+, 1}+\epsilon \mathfrak{a}_{+,-1} \pm \mathfrak{a}_{-, 1} \pm \epsilon \mathfrak{a}_{-,-1}\right], \quad L_{ \pm}^{0}=\frac{1}{2}\left[\mathfrak{a}_{+, 0} \pm \mathfrak{a}_{-, 0}\right] .
$$

Inserting these relations in (92) and using (30) we find

$$
\Theta_{+,+1} \mathbf{A}=\Theta_{+, 0} D^{-1 / 2} \dot{\mathbf{E}}, \quad \Theta_{+,-1} \mathbf{E}=-\Theta_{+, 0} D^{-1 / 2} \dot{\mathbf{A}} .
$$

These in turn allow us to express the most general physically admissible inner product on the space $\mathcal{V}$ of the Proca fields, namely, (93), as

$$
\begin{aligned}
\left(\left(A, A^{\prime}\right)\right)_{\{\mathfrak{a}\}}:= & \frac{\kappa}{2 \mathcal{M}}\left[<\mathbf{A}\left(x^{0}\right), \Theta_{+, 0} D^{-1 / 2} \dot{\mathbf{E}}^{\prime}\left(x^{0}\right)>-<\mathbf{E}\left(x^{0}\right), \Theta_{+, 0} D^{-1 / 2} \dot{\mathbf{A}}^{\prime}\left(x^{0}\right)>\right. \\
& \left.-i\left\{<\mathbf{A}\left(x^{0}\right), \Theta_{-, 0} \mathbf{E}^{\prime}\left(x^{0}\right)>-<\mathbf{E}\left(x^{0}\right), \Theta_{-, 0} \mathbf{A}^{\prime}\left(x^{0}\right)>\right\}\right],
\end{aligned}
$$

where, according to Eq. (92),

$$
\Theta_{ \pm, 0}=\left[L_{ \pm}^{+}-L_{ \pm}^{0}\right] \mathfrak{h}^{2}+L_{ \pm}^{-} \mathfrak{h}+L_{ \pm}^{0} .
$$

Note that $L_{+}^{+}$is a positive number and we can absorb it in the definition of the field. Therefore, the inner product (98) actually involves five nontrivial free parameters. Endowing $\mathcal{V}$ with this inner 
product and Cauchy completing the resulting inner product space, we obtain a separable Hilbert space which we denote by $\mathcal{H}_{\{\mathfrak{a}\}}$.

We can express the inner product (98) in terms of the indefinite Proca inner product (41). First, we use the unitary operator $U_{x_{0}^{0}}: \mathcal{H}_{\{\mathfrak{a}\}} \rightarrow \widetilde{\mathcal{K}}$ to find an expression for the action of the helicity operator $\mathfrak{H}:=U_{x_{0}^{0}}^{-1} \Lambda U_{x_{0}^{0}}$ on a Proca field $A \in \mathcal{H}_{\{\mathfrak{a}\}}$. $[\mathfrak{H} A]\left(x^{0}\right)$ is a Proca field whose components can be evaluated using (53), (33), and (17). This gives

$$
[\mathfrak{H} A]\left(x^{0}\right)=\left(0, \mathfrak{h} \mathbf{A}\left(x^{0}\right)\right) .
$$

Because $[\Lambda, H]=0$ and $\mathfrak{H}$ and $\mathrm{h}$ are, respectively, related via a similarity transformation to $\Lambda$ and $H$, we have $[\mathfrak{H}, \mathrm{h}]=0$. This together with the identity $\mathfrak{H}^{3}=\mathfrak{H}$ suggest to use $\mathfrak{H}$ to split $\mathcal{V}$ into the subspaces $\mathcal{V}_{ \pm 1}$ and $\mathcal{V}_{0}$ of \pm 1 - and 0-helicity Proca fields according to $\mathcal{V}_{h}:=\left\{A_{h} \in \mathcal{V} \mid \mathfrak{H} A_{h}={ }_{h} A_{h}\right\}$, where $h \in\{-1,0,+1\}$. We can use $\mathfrak{H}$ to define the \pm 1 - and 0 -helicity components of the Proca fields $A \in \mathcal{V}$ according to

$$
A_{ \pm 1}:=\frac{1}{2}\left(\mathfrak{H}^{2} A \pm \mathfrak{H} A\right) \in \mathcal{V}_{ \pm 1}, \quad A_{0}:=\left(A-\mathfrak{H}^{2} A\right) \in \mathcal{V}_{0}
$$

Clearly, $A=A_{+1}+A_{-1}+A_{0}$ which implies $\mathcal{V}=\mathcal{V}_{+1} \oplus \mathcal{V}_{-1} \oplus \mathcal{V}_{0}$. Restricting the inner product (98) to $\mathcal{V}_{ \pm 1}$ and $\mathcal{V}_{0}$ (and Cauchy completing the resulting inner product spaces), we obtain Hilbert subspaces $\mathcal{H}_{\{\mathfrak{a}\} \pm 1}$ and $\mathcal{H}_{\{\mathfrak{a}\} 0}$ of $\mathcal{H}_{\{\mathfrak{a}\}}$.

Next we recall that $\mathfrak{H}$ is obtained via a unitary similarity transformation from the Hermitian operator $\Lambda$. This in turn implies

$$
\left(\left(A, \mathfrak{H} A^{\prime}\right)\right)_{\{\mathfrak{a}\}}=\left(\left(\mathfrak{H} A, A^{\prime}\right)\right)_{\{\mathfrak{a}\}} .
$$

Therefore, $\mathfrak{H}$ is a Hermitian operator acting is $\mathcal{H}_{\{\mathfrak{a}\}}$. It is a physical observable that measures the helicity of $A$. Furthermore, in light of (102) and (101),

$$
\left(\left(A_{+1}, A_{-1}\right)\right)_{\{\mathfrak{a}\}}=\left(\left(A_{+1}, A_{0}\right)\right)_{\{\mathfrak{a}\}}=\left(\left(A_{-1}, A_{0}\right)\right)_{\{\mathfrak{a}\}}=0, \quad \forall A_{h} \in \mathcal{V}_{h}, h \in\{-1,0,+1\} .
$$

These relations show that $\mathcal{H}_{\{\mathfrak{a}\}}=\mathcal{H}_{\{\mathfrak{a}\}+1} \oplus \mathcal{H}_{\{\mathfrak{a}\}-1} \oplus \mathcal{H}_{\{\mathfrak{a}\} 0}$. Also, as we have shown in Sec. III, the chirality operator $\mathrm{C}$ is a Hermitian involution acting in $\mathcal{H}_{\{a\}}$ which decomposes the Hilbert space $\mathcal{H}_{\{\mathfrak{a}\}}$ into its \pm -energy Hilbert subspaces $\mathcal{H}_{\{\mathfrak{a}\}_{ \pm}}$. Thus, using the operators $\mathfrak{H}$ and $\mathrm{C}$, we can decompose the Hilbert space into six mutually orthogonal subspaces $\mathcal{H}_{\{\mathfrak{a}\}(\epsilon, h)}$, where $\epsilon=\{+,-\}$ and $h \in\{-1,0,+1\}$. Employing this decomposition of $A$ in (98), we find

$$
\left(\left(A, A^{\prime}\right)\right)_{\{\mathfrak{a}\}}=\sum_{\epsilon= \pm} \sum_{h=0, \pm 1} \epsilon \mathfrak{a}_{\epsilon, h}\left(\left(A_{\epsilon, h}, A_{\epsilon, h}^{\prime}\right)\right)_{\Sigma_{3}} .
$$

Therefore, (98) is a linear combination of the indefinite inner products (41) of the components $A_{\epsilon, h}$ of $A$ with suitable non-negative coefficients. Note that the decomposition of the fields into the helicity components (101) is clearly frame dependent and so (103) hides the Lorentz covariance of the theory. But the inner product (98) does not involve the explicit splitting of the fields into $(\epsilon, h)$-components.

In the remainder of this section, we obtain the Foldy transformation associated with the general inner product (98). First, consider the invertible linear operator $\mathcal{A}$ of (84). This is a unitary operator mapping $\widetilde{\mathcal{K}}$ onto $\mathcal{K}$, for it satisfies, for all $\xi, \zeta \in \widetilde{\mathcal{K}}$,

$$
\langle\langle\mathcal{A} \xi, \mathcal{A} \zeta\rangle\rangle_{\eta_{+}}=\left\langle\mathcal{A} \xi, \eta_{+} \mathcal{A} \zeta\right\rangle=\left\langle\xi, \mathcal{A}^{\dagger} \eta_{+} \mathcal{A} \zeta\right\rangle=\langle\langle\xi, \zeta\rangle\rangle_{\tilde{\eta}_{+}} .
$$

We can find the explicit form of $\mathcal{A}$ and $\mathcal{A}^{-1}$ by substituting (44) and (46) in (84). This gives

$$
\mathcal{A}=\left[F_{-}^{+} \sigma_{3} M-F_{-}^{0} \sigma_{3} N+\left(F_{+}^{+}-F_{+}^{0}\right) \sigma_{0}\right] \mathfrak{h}^{2}+\left[F_{-}^{-} \sigma_{3} M+F_{+}^{-} \sigma_{0}\right] \mathfrak{h}+F_{-}^{0} \sigma_{3} N+F_{+}^{0} \sigma_{0},
$$




$$
\mathcal{A}^{-1}=\left[\widetilde{F}_{-}^{+} \sigma_{3} M-\widetilde{F}_{-}^{0} \sigma_{3} N+\left(\widetilde{F}_{+}^{+}-\widetilde{F}_{+}^{0}\right) \sigma_{0}\right] \mathfrak{h}^{2}+\left[\widetilde{F}_{-}^{-} \sigma_{3} M+\widetilde{F}_{+}^{-} \sigma_{0}\right] \mathfrak{h}+\widetilde{F}_{-}^{0} \sigma_{3} N+\widetilde{F}_{+}^{0} \sigma_{0},
$$

where

$$
\begin{gathered}
F_{ \pm}^{\epsilon}:=\frac{1}{2}\left[Z_{\epsilon}^{+} \pm Z_{\epsilon}^{-}\right], \quad F_{ \pm}^{0}:=\frac{1}{2}\left[\alpha_{+, 0} \pm \alpha_{-, 0}\right], \\
\widetilde{F}_{ \pm}^{\epsilon}:=\frac{1}{2}\left[\widetilde{Z}_{\epsilon}^{+} \pm \tilde{Z}_{\epsilon}\right], \quad \widetilde{F}_{ \pm}^{0}:=\frac{1}{2}\left[\alpha_{+, 0}^{-1} \pm \alpha_{-, 0}^{-1}\right], \\
Z_{ \pm}^{\epsilon}:=\frac{1}{2}\left[\alpha_{\epsilon, 1} \pm \alpha_{\epsilon,-1}\right], \quad \widetilde{Z}_{ \pm}^{\epsilon}:=\frac{1}{2}\left[\alpha_{\epsilon, 1}^{-1} \pm \alpha_{\epsilon,-1}^{-1}\right] .
\end{gathered}
$$

Next, we define $\tilde{\rho}: \widetilde{\mathcal{K}} \rightarrow \mathcal{H}^{\prime}$ and $\mathcal{U}_{\{\mathfrak{a}\}}: \mathcal{H}_{\{\mathfrak{a}\}} \rightarrow \mathcal{H}^{\prime}$ by

$$
\begin{gathered}
\tilde{\rho}:=\rho \mathcal{A}, \\
\mathcal{U}_{\{\mathrm{a}\}}=\tilde{\rho} U_{x_{0}^{0}} .
\end{gathered}
$$

In view of $\rho^{\dagger}=\rho=\sqrt{\eta_{+}}$and (85), $\tilde{\rho}$ is a unitary transformation mapping $\widetilde{\mathcal{K}}$ onto $\mathcal{H}^{\prime}$,

$$
\langle\widetilde{\rho} \xi, \tilde{\rho} \zeta\rangle=\langle\xi, \widetilde{\rho} \dagger \tilde{\rho} \zeta\rangle=\left\langle\xi, \mathcal{A}^{\dagger} \eta_{+} \mathcal{A} \zeta\right\rangle=\langle\langle\xi, \zeta\rangle\rangle_{\tilde{\eta}_{+}} .
$$

This in turn implies that $\mathcal{U}_{\{a\}}: \mathcal{H}_{\{\mathfrak{a}\}} \rightarrow \mathcal{H}^{\prime}$ is also a unitary transformation,

$$
\left\langle\mathcal{U}_{\{\mathfrak{a}\}} A, \mathcal{U}_{\{\mathfrak{a}\}} A^{\prime}\right\rangle=\left(\left(A, A^{\prime}\right)\right)_{\{\mathfrak{a}\}}, \quad \forall A, A^{\prime} \in \mathcal{H}_{\{\mathfrak{a}\}} .
$$

We can compute the explicit form of the unitary operator $\mathcal{U}_{\{a\}}$ and its inverse. This requires computing $\widetilde{\rho}$ and its inverse. Substituting (61), (62), (105), and (106) in (110), we have

$$
\tilde{\rho}=\frac{1}{2 \mathcal{M} \sqrt{\gamma}}\left(\begin{array}{ll}
\tilde{\rho}_{+,+} & \tilde{\rho}_{-,+} \\
\tilde{\rho}_{-,-} & \tilde{\rho}_{+,-}
\end{array}\right), \quad \widetilde{\rho}^{-1}=\frac{1}{2 \mathcal{M} \sqrt{\gamma}}\left(\begin{array}{cc}
\tilde{\rho}_{+,+}^{\text {inv }} & -\tilde{\rho}_{-,-}^{\text {inv }} \\
-\tilde{\rho}_{-,+}^{\text {inv }} & \tilde{\rho}_{+,-}^{\text {inv }}
\end{array}\right),
$$

where the entries are the following operators that act in $\tilde{\mathcal{H}}$ :

$$
\begin{aligned}
& \tilde{\rho}_{\epsilon, \epsilon^{\prime}}:=\mathcal{M}\left(\gamma D^{1 / 4}+\epsilon D^{-1 / 4}\right)\left[Z_{+}^{\epsilon^{\prime}} \mathfrak{h}^{2}+Z_{-}^{\epsilon^{\prime}} \mathfrak{h}\right]+\alpha_{\epsilon^{\prime}, 0}\left(\gamma \mathcal{M}^{2} D^{-1 / 4}+\epsilon D^{1 / 4}\right)\left[\lambda_{0}-\mathfrak{h}^{2}\right], \\
& \tilde{\rho}_{\epsilon, \epsilon^{\prime}}^{\text {inv }}:=\mathcal{M}\left(\gamma D^{1 / 4}+\epsilon D^{-1 / 4}\right)\left[\widetilde{Z}_{+}^{\epsilon^{\prime}} \mathfrak{h}^{2}+\widetilde{Z}_{-}^{\epsilon^{\prime}} \mathfrak{h}\right]+\alpha_{\epsilon^{\prime}, 0}^{-1}\left(\gamma \mathcal{M}^{2} D^{-1 / 4}+\epsilon D^{1 / 4}\right)\left[\lambda_{0}-\mathfrak{h}^{2}\right] .
\end{aligned}
$$

Next, we use (111), (114), (115), (53), and (35), to find, for all $A \in \mathcal{H}$,

$$
\mathcal{U}_{\{\mathfrak{a}\}} A=\frac{1}{2} \sqrt{\frac{\kappa}{\mathcal{M}}}\left(\begin{array}{l}
\mathfrak{U}_{+,+} \mathbf{A}\left(x_{0}^{0}\right)-i \mathfrak{U}_{+,-} \mathbf{E}\left(x_{0}^{0}\right) \\
\mathfrak{U}_{-,+} \mathbf{A}\left(x_{0}^{0}\right)+i \mathfrak{U}_{-,-} \mathbf{E}\left(x_{0}^{0}\right)
\end{array}\right),
$$

where the operators $\mathfrak{U}_{\epsilon, \epsilon^{\prime}}: \widetilde{\mathcal{H}} \rightarrow \widetilde{\mathcal{H}}$ are given by

$$
\mathfrak{U}_{\epsilon, \epsilon^{\prime}}=\left[Z_{+}^{\epsilon} D^{\epsilon^{\prime} / 4}-\mathcal{M}^{\epsilon^{\prime}} \alpha_{\epsilon, 0} D^{-\epsilon^{\prime} / 4}\right] \mathfrak{h}^{2}+Z_{-}^{\epsilon} D^{\epsilon^{\prime} / 4} \mathfrak{h}+\mathcal{M}^{\epsilon^{\prime}} \alpha_{\epsilon, 0} D^{-\epsilon^{\prime} / 4} .
$$

Again the arbitrary parameter $\gamma$ drops out of the calculations. $\mathcal{U}_{\{\mathfrak{a}\}}^{-1}=U_{x_{0}^{0}}^{-1} \widetilde{\rho}^{-1}$ is also easy to calculate. Let $\xi \in \mathcal{H}^{\prime}$ be a six-component vector [as in (51)] with components $\boldsymbol{\xi}^{1}$ and $\boldsymbol{\xi}^{2}$. Then in view of (117), $\mathcal{U}_{\{\mathfrak{a}\}}^{-1} \xi$ is the Proca field $A \in \mathcal{H}_{\{\mathfrak{a}\}}$ satisfying the following initial conditions:

$$
A^{0}\left(x_{0}^{0}\right)=\frac{D^{-1 / 4}}{\sqrt{\mathcal{M} \kappa}} \mathfrak{K} \cdot\left(\frac{\xi^{1}}{\alpha_{+, 0}}-\frac{\xi^{2}}{\alpha_{-, 0}}\right), \quad \dot{A}^{0}\left(x_{0}^{0}\right)=-i \frac{D^{1 / 4}}{\sqrt{\mathcal{M} \kappa}} \mathfrak{K} \cdot\left(\frac{\xi^{1}}{\alpha_{+, 0}}+\frac{\xi^{2}}{\alpha_{-, 0}}\right),
$$




$$
\mathbf{A}\left(x_{0}^{0}\right)=\sqrt{\frac{\bar{M}}{\kappa}}\left(\mathfrak{U}_{+,+}^{-1} \xi^{1}+\mathfrak{U}_{-,+}^{-1} \xi^{2}\right), \quad \dot{\mathbf{A}}\left(x_{0}^{0}\right)=-i \sqrt{\frac{\mathcal{M}}{\kappa}} D^{1 / 2}\left(\mathfrak{U}_{+,+}^{-1} \xi^{1}-\mathfrak{U}_{-,+}^{-1} \xi^{2}\right),
$$

where $\mathfrak{U}_{\epsilon,+}^{-1}$ is the inverse of $\mathfrak{U}_{\epsilon,+}$ given by

$$
\mathfrak{U}_{\epsilon,+}^{-1}=\left[\tilde{Z}_{+}^{\epsilon} D^{-1 / 4}-\frac{D^{1 / 4}}{\mathcal{M} \alpha_{\epsilon, 0}}\right] \mathfrak{h}^{2}+\tilde{Z}_{-}^{\epsilon} D^{-1 / 4} \mathfrak{h}+\frac{D^{1 / 4}}{\mathcal{M} \alpha_{\epsilon, 0}},
$$

and we have made use of (15), (30), (33), (35), (53), and (114)-(116). By virtue of (17), for all $x_{0}^{0} \in \mathrm{R}$, we have

$$
\begin{gathered}
A^{0}\left(x^{0}\right)=\frac{D^{-1 / 4}}{\sqrt{\mathcal{M} \kappa}}\left[e^{-i\left(x^{0}-x_{0}^{0}\right) D^{1 / 2}} \mathfrak{K} \cdot \frac{\xi^{1}}{\alpha_{+, 0}}-e^{i\left(x^{0}-x_{0}^{0}\right) D^{1 / 2}} \mathfrak{K} \cdot \frac{\boldsymbol{\xi}^{2}}{\alpha_{-, 0}}\right], \\
\mathbf{A}\left(x^{0}\right)=\sqrt{\frac{\mathcal{M}}{\kappa}}\left[e^{-i\left(x^{0}-x_{0}^{0}\right) D^{1 / 2}} \mathfrak{U}_{+,+}^{-1} \xi^{1}+e^{i\left(x^{0}-x_{0}^{0}\right) D^{1 / 2}} \mathfrak{U}_{-,+}^{-1} \boldsymbol{\xi}^{2}\right] .
\end{gathered}
$$

Next we recall that since $[H, \mathcal{A}]=0$, we have $\mathrm{h}_{\{\mathfrak{a}\}}=\mathrm{h}$. Therefore, in light of the above analysis, the pairs $\left(\mathcal{H}_{\{\mathrm{a}\}}, \mathrm{h}\right),(\tilde{\mathcal{K}}, H)$, and $\left(\mathcal{H}^{\prime}, H^{\prime}\right)$ are unitarily equivalent; they represent the same quantum system.

Finally, we give an alternative form of the Foldy transformation (117). In view of (83) and $\mathfrak{U}_{\epsilon,-}^{-1} \mathbf{E}=-\mathfrak{U}_{\epsilon,+} D^{-1 / 2} \dot{\mathbf{A}}$, we have

$$
\mathcal{U}_{\{\mathfrak{a}\}} A=\sqrt{\frac{\kappa}{\mathcal{M}}}\left(\begin{array}{l}
\mathfrak{U}_{+,+} \mathbf{A}_{+}\left(x_{0}^{0}\right) \\
\mathfrak{U}_{-,+} \mathbf{A}_{-}\left(x_{0}^{0}\right)
\end{array}\right) .
$$

In particular, setting $\alpha_{\epsilon, h}=1$, for all $\epsilon \in\{+,-\}$ and $h \in\{-1,0,+1\}$, yields

$$
\mathcal{U} A=\sqrt{\frac{\kappa}{\mathcal{M}}} \mathfrak{U}\left(\begin{array}{l}
\mathbf{A}_{+}\left(x_{0}^{0}\right) \\
\mathbf{A}_{-}\left(x_{0}^{0}\right)
\end{array}\right),
$$

where $\mathbf{A}_{ \pm}$is \pm -energy component of Proca field. These equations show that $\mathfrak{U}_{\epsilon,+} \mathbf{A}_{\epsilon}$ (especially $\left.\mathfrak{U} \mathbf{A}_{\epsilon}\right)$ satisfy the Foldy equation ${ }^{17,16}$

$$
i \partial_{0} \mathfrak{U}_{\epsilon,+} \mathbf{A}_{\epsilon}=\epsilon D^{1 / 2} \mathfrak{U}_{\epsilon,+} \mathbf{A}_{\epsilon} .
$$

\section{CONSERVED CURRENT DENSITY}

The relativistic and time-translation invariance of the positive-definite inner products (98) suggest the existence of an associated conserved four-vector current density $J_{\{\mathfrak{a}\}}^{\mu}$. In this section we use the approach of Ref. 6 to compute $J_{\{\mathfrak{a}\}}^{\mu}$ for the case $\mathfrak{a}_{\epsilon, h}=1$ and obtain a manifestly covariant expression for $J_{\{\mathfrak{a}\}}^{\mu}$ with $\mathfrak{a}_{\epsilon, h}=1$ which for brevity we denote by $J^{\mu}$.

According to (98), for all $A \in \mathcal{V}$,

$$
\begin{aligned}
((A, A))_{\{\mathfrak{a}\}}= & \frac{\kappa}{2 \mathcal{M}} \int_{\mathbb{R}^{3}} d^{3} \mathbf{x}\left\{\mathbf{A}\left(x^{0}, \mathbf{x}\right)^{*} \cdot\left(\Theta_{+, 0} D^{-1 / 2} \dot{\mathbf{E}}\left(x^{0}, \mathbf{x}\right)\right)-\mathbf{E}\left(x^{0}, \mathbf{x}\right)^{*} \cdot\left(\Theta_{+, 0} D^{-1 / 2} \dot{\mathbf{A}}\left(x^{0}, \mathbf{x}\right)\right)\right. \\
& \left.-i\left[\mathbf{A}\left(x^{0}, \mathbf{x}\right)^{*} \cdot\left(\Theta_{-, 0} \mathbf{E}\left(x^{0}, \mathbf{x}\right)\right)-\mathbf{E}\left(x^{0}, \mathbf{x}\right)^{*} \cdot\left(\Theta_{-, 0} \mathbf{A}\left(x^{0}, \mathbf{x}\right)\right)\right]\right\} .
\end{aligned}
$$

In analogy with nonrelativistic QM, we define the 0 -component of the current density $J_{\{\mathfrak{a}\}}^{\mu}$ associated with $A$ as the integrand in (127), i.e., 


$$
\begin{aligned}
J_{\{\mathfrak{a}\}}^{0}(x):= & \frac{\kappa}{2 \mathcal{M}}\left\{\mathbf{A}(x)^{*} \cdot\left(\Theta_{+, 0} D^{-1 / 2} \dot{\mathbf{E}}(x)\right)-\mathbf{E}(x)^{*} \cdot\left(\Theta_{+, 0} D^{-1 / 2} \dot{\mathbf{A}}(x)\right)\right. \\
& \left.-i\left[\mathbf{A}(x)^{*} \cdot\left(\Theta_{-, 0} \mathbf{E}(x)\right)-\mathbf{E}(x)^{*} \cdot\left(\Theta_{-, 0} \mathbf{A}(x)\right)\right]\right\} .
\end{aligned}
$$

Setting $\mathfrak{a}_{\epsilon, h}=1$ for all $\epsilon \in\{+,-\}$ and $h \in\{-1,0,+1\}$ yields

$$
J^{0}(x):=\frac{\kappa}{2 \mathcal{M}}\left\{\mathbf{A}(x)^{*} \cdot D^{-1 / 2} \dot{\mathbf{E}}(x)-\mathbf{E}(x)^{*} \cdot D^{-1 / 2} \dot{\mathbf{A}}(x)\right\} .
$$

In order to obtain the spatial components of $J^{\mu}$, we follow the procedure outlined in Ref. 6. Namely, we perform an infinitesimal Lorentz boost transformation that changes the reference frame to the one moving with a velocity $\mathbf{v}$,

$$
x^{0} \rightarrow x^{\prime 0}=x^{0}-\boldsymbol{\beta} \cdot \mathbf{x}, \quad \mathbf{x} \rightarrow \mathbf{x}^{\prime}=\mathbf{x}-\boldsymbol{\beta} x^{0},
$$

where $\boldsymbol{\beta}:=\mathbf{v} / c$. Assuming that $J^{\mu}$ is indeed a four-vector field and neglecting the second and higher order terms in powers of the components of $\boldsymbol{\beta}$, we then find

$$
J^{0}(x) \rightarrow J^{\prime 0}\left(x^{\prime}\right)=J^{0}(x)-\boldsymbol{\beta} \cdot \mathbf{J}(x) .
$$

Next, we use (129) to obtain

$$
J^{\prime 0}\left(x^{\prime}\right):=\frac{\kappa}{2 \mathcal{M}}\left\{\mathbf{A}^{\prime}\left(x^{\prime}\right)^{*} \cdot D^{\prime-1 / 2} \dot{\mathbf{E}}^{\prime}\left(x^{\prime}\right)-\mathbf{E}^{\prime}\left(x^{\prime}\right)^{*} \cdot D^{\prime-1 / 2} \dot{\mathbf{A}}^{\prime}\left(x^{\prime}\right)\right\}
$$

where $x^{\prime}:=\left(x^{\prime 0}, \mathbf{x}^{\prime}\right), D^{\prime}=-\nabla^{\prime 2}+\mathcal{M}^{2}, \mathbf{E}^{\prime}\left(x^{\prime}\right)=-\dot{\mathbf{A}}^{\prime}\left(x^{\prime}\right)-\nabla^{\prime} A^{\prime 0}\left(x^{\prime}\right)$, and $\dot{\mathbf{A}}^{\prime}\left(x^{\prime}\right):=\partial \mathbf{A}^{\prime}\left(x^{\prime}\right) / \partial x^{\prime 0}$. This reduces the determination of $\mathbf{J}$ to expressing the right-hand side of (132) in terms of the quantities associated with the original (unprimed) frame and comparing the resulting expression with (131).

Under the transformation (130), the four-vector $A=\left(A^{0}, \mathbf{A}\right)$ transforms as

$$
A^{0}(x) \rightarrow A^{\prime 0}\left(x^{\prime}\right)=A^{0}(x)-\boldsymbol{\beta} \cdot \mathbf{A}(x), \quad \mathbf{A}(x) \rightarrow \mathbf{A}^{\prime}\left(x^{\prime}\right)=\mathbf{A}(x)-\boldsymbol{\beta} A^{0}(x) .
$$

Therefore, in view of (130), we can easily obtain the transformation rule for $\mathbf{E}$. The result is

$$
\mathbf{E}(x) \rightarrow \mathbf{E}^{\prime}\left(x^{\prime}\right)=\mathbf{E}(x)+\boldsymbol{\beta} \times(\boldsymbol{\nabla} \times \mathbf{A}(x)) .
$$

Because the chirality operator $\mathrm{C}=i D^{-1 / 2} \partial_{0}$ is Lorentz invariant, ${ }^{6}$

$$
D^{\prime-1 / 2} \partial_{0}^{\prime}=D^{-1 / 2} \partial_{0} .
$$

Substituting (133)-(135) in (132), making use of (131), we obtain

$$
\begin{aligned}
J^{i}(x)= & \frac{\kappa}{2 \mathcal{M}}\left\{A^{*}(x) \cdot \partial^{i} D^{-1 / 2} \dot{A}(x)-\left[\partial^{i} A(x)^{*}\right] \cdot D^{-1 / 2} \dot{A}(x)-\left[A^{*}(x) \cdot \partial\right] D^{-1 / 2} \dot{A}^{i}(x)\right. \\
& \left.+\left[D^{-1 / 2} \dot{A}(x) \cdot \partial\right] A^{i}(x)^{*}\right\},
\end{aligned}
$$

where $\partial:=\left(\partial^{0}, \nabla\right)$ and for any two four-vectors $v_{1}$ and $v_{2}, v_{1} \cdot v_{2}:=v_{1}^{\mu} v_{2 \mu}$. This relation suggests

$$
\begin{aligned}
J^{\mu}(x)= & \frac{\kappa}{2 \mathcal{M}}\left\{A^{*}(x) \cdot \partial^{\mu} D^{-1 / 2} \dot{A}(x)-\left[\partial^{\mu} A(x)^{*}\right] \cdot D^{-1 / 2} \dot{A}(x)-\left[A^{*}(x) \cdot \partial\right] D^{-1 / 2} \dot{A}^{\mu}(x)\right. \\
& \left.+\left[D^{-1 / 2} \dot{A}(x) \cdot \partial\right] A^{\mu}(x)^{*}\right\} .
\end{aligned}
$$

It is not difficult to check (using the Proca equation) that the expression for $J^{0}$ obtained using this equation agrees with the one given in (129).

We can use (2) and (82) to further simplify (137). This yields 


$$
J^{\mu}(x)=\frac{i \kappa}{2 \mathcal{M}}\left\{A_{\nu}(x)^{*} F_{c}^{\nu \mu}(x)-F^{\nu \mu}(x)^{*} A_{c \nu}(x)\right\}
$$

where $F_{c}^{\mu \nu}:=\partial^{\mu} A_{c}^{\nu}-\partial^{\nu} A_{c}^{\mu}$. The current density $J^{\mu}$ which is generally complex-valued has the following remarkable properties.

(1) The expression (138) for $J^{\mu}$ is manifestly covariant; since $A$ and $A_{c}$ are four-vector fields, so is $J^{\mu}$.

(2) Using the fact that both $A$ and $A_{c}$ satisfy the Proca equation (4), one easily checks that $J^{\mu}$ satisfies the following continuity equation:

$$
\partial_{\mu} J^{\mu}=0
$$

Hence it is a conserved current density.

Next, we use (138) to derive a manifestly covariant expression for the inner product (55) on the space of solutions of the Proca equation (11). The result is

$$
\left(\left(A, A^{\prime}\right)\right)=\frac{i \kappa}{2 \mathcal{M}} \int_{\sigma} d \sigma(x) n_{\mu}(x)\left\{A_{\nu}(x)^{*} F_{c}^{\prime \nu \mu}(x)-F^{\nu \mu}(x)^{*} A_{c \nu}^{\prime}(x)\right\},
$$

where $\sigma$ is an arbitrary spacelike (Cauchy) hypersurface of the Minkowski space with volume element $d \sigma$ and unit (future) timelike normal four-vector $n^{\mu}$. Note that in deriving (140) we have also made an implicit use of the fact that any inner product is uniquely determined by the corresponding norm. 47

Using the same approach we have calculated $J_{\{\mathfrak{a}\}}^{\mu}$ for $\mathfrak{a}_{\epsilon, h} \neq 1$ and checked that indeed it is a conserved complex-valued four-vector field. But we were not able to obtain a manifestly covariant expression for $J_{\{\mathfrak{a}\}}^{\mu}$ in this case. As the expression for $J_{\{\mathfrak{a}\}}^{\mu}$ is highly complicated we do not present it here.

\section{PHYSICAL OBSERVABLES AND WAVE FUNCTIONS FOR PROCA FIELDS}

The unitary equivalence of the representations $\left(\mathcal{H}_{\{\mathfrak{a}\}}, \mathrm{h}\right),(\widetilde{\mathcal{K}}, H)$, and $\left(\mathcal{H}^{\prime}, H^{\prime}\right)$ for the quantum system describing the Proca fields allows for the construction of the observables of this system using any of these representations. Because $\mathcal{H}^{\prime}=\tilde{\mathcal{H}} \oplus \tilde{\mathcal{H}}$ and $\tilde{\mathcal{H}}=L^{2}\left(\mathbb{R}^{3}\right) \oplus L^{2}\left(\mathbb{R}^{3}\right) \oplus L^{2}\left(\mathbb{R}^{3}\right)$, the Foldy representation $\left(\mathcal{H}^{\prime}, H^{\prime}\right)$ is more convenient for this purpose. In this section we construct the observables in this representation and use the unitary map $\mathcal{U}_{\{\mathfrak{a}\}}: \mathcal{H}_{\{\mathfrak{a}\}} \rightarrow \mathcal{H}^{\prime}$ to obtain their form in the standard (covariant) representation $\left(\mathcal{H}_{\{\mathfrak{a}\}}, \mathrm{h}\right)$. Again we follow closely the approach used in Ref. 27 to construct the observables for $\mathrm{KG}$ fields.

In the Foldy representation, we introduce the following set of basic observables:

$$
\mathbf{X}_{\mathfrak{m}}^{\prime}:=\mathbf{x} \otimes \Sigma_{\mathfrak{m}}, \quad \mathbf{P}_{\mathfrak{m}}^{\prime}:=\mathbf{p} \otimes \Sigma_{\mathfrak{m}}, \quad \mathcal{S}_{\mathfrak{m}}^{\prime}:=1 \otimes \Sigma_{\mathfrak{m}}
$$

where $\mathbf{x}, \mathbf{p}=\hbar \mathfrak{K}$, and 1 are, respectively, the position, momentum, and identity operators acting in $L^{2}\left(\mathbb{R}^{3}\right), \mathfrak{m} \in\{0,1,2, \ldots, 35\}$, and $\Sigma_{\mathfrak{m}}$ 's are given in Eq. (9). In the following, we will omit " $1 \otimes$ " for brevity. In particular, we will identify $\mathcal{S}_{\mathfrak{m}}^{\prime}$ with $\Sigma_{\mathfrak{m}}$.

As we mentioned in Sec. I, the operators $\mathbf{X}_{0}^{\prime}, \Sigma_{3}, \Sigma_{12}, \Sigma_{15}, \Sigma_{32}$, and $\Sigma_{35}$ form a maximal commuting set of observables acting in $\mathcal{H}^{\prime}$. In particular, we can use their common eigenvectors, namely,

$$
\xi_{\mathbf{x}}^{(\epsilon, s)}:=|\mathbf{x}\rangle \otimes e_{\epsilon, s}, \quad \mathbf{x} \in \mathbb{R}^{3}, \quad \epsilon \in\{-,+\}, \quad s \in\{-1,0,+1\},
$$

to construct a basis of $\mathcal{H}^{\prime}$. In (142), $e_{\epsilon, s}$ are the vectors defined in (10) and $|\mathbf{x}\rangle$ are the $\delta$-function normalized position kets satisfying $\mathbf{x}|\mathbf{x}\rangle=\mathbf{x}|\mathbf{x}\rangle,\left\langle\mathbf{x} \mid \mathbf{x}^{\prime}\right\rangle=\delta^{3}\left(\mathbf{x}-\mathbf{x}^{\prime}\right)$, and $\int_{\mathrm{R}^{3}} d^{3} \mathbf{x}|\mathbf{x}\rangle\langle\mathbf{x}|=1$. It is easy to see that indeed $\mathbf{X}_{0}^{\prime} \xi_{\mathbf{x}}^{(\epsilon, s)}=\mathbf{x} \xi_{\mathbf{x}}^{(\epsilon, s)}, \Sigma_{3} \xi_{\mathbf{x}}^{(\epsilon, s)}=\epsilon \xi_{\mathbf{x}}^{(\epsilon, s)}$, and $\Sigma_{12} \xi_{\mathbf{x}}^{(\epsilon, s)}=s \xi_{\mathbf{x}}^{(\epsilon, s)}$. Furthermore, 


$$
\left\langle\xi_{\mathbf{x}}^{(\epsilon, s)}, \xi_{\mathbf{x}^{\prime}}^{\left(\epsilon^{\prime}, s^{\prime}\right)}\right\rangle=\delta_{\epsilon, \epsilon^{\prime}} \delta_{s, s^{\prime}} \delta^{3}\left(\mathbf{x}-\mathbf{x}^{\prime}\right), \quad \sum_{\epsilon= \pm} \sum_{s=0, \pm 1} \int_{\mathbb{R}^{3}} d^{3} \mathbf{x}\left|\xi_{\mathbf{x}}^{(\epsilon, s)}\right\rangle\left\langle\xi_{\mathbf{x}}^{(\epsilon, s)}\right|=\Sigma_{0}
$$

We can express any six-component vector $\Psi^{\prime} \in \mathcal{H}^{\prime}$ in the basis $\left\{\xi_{\mathbf{x}}^{(\epsilon, s)}\right\}$ according to

$$
\Psi^{\prime}=\sum_{\epsilon= \pm} \sum_{s=0, \pm 1} \int_{\mathbb{R}^{3}} d^{3} \mathbf{x} f(\epsilon, s, \mathbf{x}) \xi_{\mathbf{x}}^{(\epsilon, s)},
$$

where $f:\{-,+\} \times\{-1,0,+1\} \times \mathbb{R}^{3} \rightarrow \mathrm{C}$ is the wave function associated with $\Psi^{\prime}$ in the position representation, i.e.,

$$
f(\epsilon, s, \mathbf{x}):=\left\langle\xi_{\mathbf{x}}^{(\epsilon, s)}, \Psi^{\prime}\right\rangle .
$$

Also, the action of a physical observable $O^{\prime}: \mathcal{H}^{\prime} \rightarrow \mathcal{H}^{\prime}$ on the state vector $\Psi^{\prime} \in \mathcal{H}^{\prime}$ is given by

$$
O^{\prime} \Psi^{\prime}=\sum_{\epsilon= \pm} \sum_{s=0, \pm 1} \int_{\mathbb{R}^{3}} d^{3} \mathbf{x}[\hat{O} f(\epsilon, s, \mathbf{x})] \xi_{\mathbf{x}}^{(\epsilon, s)}
$$

where

$$
\hat{O} f(\epsilon, s, \mathbf{x}):=\sum_{\epsilon^{\prime}= \pm} \sum_{s^{\prime}=0, \pm 1} \int_{\mathbb{R}^{3}} d^{3} \mathbf{x}^{\prime} f\left(\epsilon^{\prime}, s^{\prime}, \mathbf{x}^{\prime}\right)\left\langle\xi_{\mathbf{x}}^{(\epsilon, s)}, O^{\prime} \xi_{\mathbf{x}^{\prime}}^{\left(\epsilon^{\prime}, s^{\prime}\right)}\right\rangle .
$$

This provides the representation of observables in terms of linear operators acting on the squareintegrable wave functions $f$.

Next, we introduce the operators

$$
\mathbf{x}_{\mathfrak{m}\{\mathfrak{a}\}}:=\mathcal{U}_{\{\mathfrak{a}\}}^{-1} \mathbf{X}_{\mathfrak{m}}^{\prime} \mathcal{U}_{\{\mathfrak{a}\}}, \quad \mathbf{p}_{\mathfrak{m}\{\mathfrak{a}\}}:=\mathcal{U}_{\{\mathfrak{a}\}}^{-1} \mathbf{P}_{\mathfrak{m}}^{\prime} \mathcal{U}_{\{\mathfrak{a}\}}, \quad s_{\mathfrak{m}\{\mathfrak{a}\}}:=\mathcal{U}_{\{\mathfrak{a}\}}^{-1} \Sigma_{\mathfrak{m}} \mathcal{U}_{\{\mathfrak{a}\}},
$$

that act in $\mathcal{H}_{\{\mathfrak{a}\}}$, and define the Proca fields

$$
A_{\{\mathfrak{a}\} \mathbf{x}}^{(\epsilon, s)}=\left(A_{\{a\} \mathbf{x}}^{0(\epsilon, s)}, \mathbf{A}_{\{a\} \mathbf{x}}^{(\epsilon, s)}:=\mathcal{U}_{\{\mathfrak{a}\}}^{-1} \xi_{\mathbf{X}}^{(\epsilon, s)},\right.
$$

which form a complete orthonormal basis of $\mathcal{H}_{\{\mathfrak{a}\}}$,

$$
\left(\left(A_{\{\mathfrak{a}\} \mathbf{x}}^{(\epsilon, s)}, A_{\{\mathfrak{a}\} \mathbf{x}^{\prime}}^{\left(\epsilon^{\prime}, s^{\prime}\right)}\right)_{\{\mathfrak{a}\}}=\delta_{\epsilon, \epsilon^{\prime}} \delta_{s, s^{\prime}} \delta^{3}\left(\mathbf{x}-\mathbf{x}^{\prime}\right), \quad \sum_{\epsilon= \pm} \sum_{s=0, \pm 1} \int_{\mathbb{R}^{3}} d^{3} \mathbf{x} \mid A_{\{\mathfrak{a}\} \mathbf{x}}^{(\epsilon, s)}\right)\left(A_{\{\mathfrak{a}\} \mathbf{x}}^{(\epsilon, s)} \mid=s_{0\{\mathfrak{a}\}} .\right.
$$

Here we have used (113), (143), and (149), $s_{0\{\mathfrak{a}\}}$ coincides with the identity operator for $\mathcal{H}_{\{\mathfrak{a}\}}$, and for all $A, A^{\prime} \in \mathcal{H}_{\{\mathfrak{a}\}}$, the operator $\left.\mid A\right)\left(A^{\prime} \mid\right.$ is defined by $\left.\mid A\right)\left(A^{\prime} \mid A^{\prime \prime}:=\left(\left(A^{\prime}, A^{\prime \prime}\right)\right)_{\{\mathfrak{a}\}} A\right.$, for any $A^{\prime \prime}$ $\in \mathcal{H}_{\{\mathfrak{a}\}}$. In view of (150) and

$$
\left(\left(A_{\{\mathfrak{a}\} \mathbf{x}}^{(\epsilon, s)}, A\right)\right)_{\{\mathfrak{a}\}}=\left\langle\mathcal{U}_{\{\mathfrak{a}\}} A_{\{\mathfrak{a}\} \mathbf{x}}^{(\epsilon, s)} \mathcal{U}_{\{\mathfrak{a}\}} A\right\rangle=\left\langle\xi_{\mathbf{x}}^{(\epsilon, s)}, \Psi^{\prime}\right\rangle=f(\epsilon, s, \mathbf{x}),
$$

we can express any Proca field $A \in \mathcal{H}_{\{\mathfrak{a}\}}$ in the basis $\left\{A_{\{\mathfrak{a}\} \mathbf{x}}^{(\epsilon, s)}\right\}$ according to

$$
A=\sum_{\epsilon= \pm} \sum_{s=0, \pm 1} \int_{\mathbb{R}^{3}} d^{3} \mathbf{x} f(\epsilon, s, \mathbf{x}) A_{\{a\} \mathbf{x}}^{(\epsilon, s)}
$$

Here we do not label the wave functions $f$ with the subscript $\{\mathfrak{a}\}$ because they do not depend on the choice of the parameter $\mathfrak{a}_{\epsilon, h}$. The proof of this assertion uses the unitary operator

$$
U_{\{\mathfrak{a}\}}:=\mathcal{U}_{\{\mathfrak{a}\}}^{-1} \mathcal{U},
$$

that maps $\mathcal{H}$ onto $\mathcal{H}_{\{\mathfrak{a}\}}$ and is identical with the one given in Ref. 6 for the KG fields. 
The physical observables $o_{\{\mathfrak{a}\}}: \mathcal{H}_{\{\mathfrak{a}\}} \rightarrow \mathcal{H}_{\{\mathfrak{a}\}}$ are uniquely specified in terms of their representation in the basis $\left\{A_{\{\mathfrak{a}\} \mathbf{x}}^{(\epsilon, s)}\right.$; for all $A \in \mathcal{H}_{\{\mathfrak{a}\}}$,

$$
o_{\{\mathfrak{a}\}} A=\sum_{\epsilon= \pm} \sum_{s=0, \pm 1} \int_{\mathbb{R}^{3}} d^{3} \mathbf{x}[\hat{O} f(\epsilon, s, \mathbf{x})] A_{\{\mathfrak{a}\} \mathbf{x}}^{(\epsilon, s)}
$$

where $\hat{O} f(\epsilon, s, \mathbf{x})$ is defined by (147). This follows from $\left(\left(A_{\{\mathfrak{a}\}_{\mathbf{x}}}^{(\epsilon, s)}, o_{\{\mathfrak{a}\}} A_{\{\mathfrak{a}\}_{\mathbf{x}^{\prime}}}^{\left(\epsilon^{\prime}, s^{\prime}\right)}\right)\right)_{\{\mathfrak{a}\}}=\left\langle\xi_{\mathbf{x}}^{(\epsilon, s)}, O^{\prime} \xi_{\mathbf{x}^{\prime}}^{\left(\epsilon^{\prime}, s^{\prime}\right)}\right\rangle$. In view of (150) and (152), we can express the transition amplitudes between two states (the inner product of two Proca fields) in the form

$$
\left(\left(A, A^{\prime}\right)\right)_{\{\mathfrak{a}\}}=\sum_{\epsilon= \pm} \sum_{s=0, \pm 1} \int_{\mathbb{R}^{3}} d^{3} \mathbf{x} f(\epsilon, s, \mathbf{x})^{*} f^{\prime}(\epsilon, s, \mathbf{x}) .
$$

More generally, for any observable $o_{\{\mathfrak{a}\}}: \mathcal{H}_{\{\mathfrak{a}\}} \rightarrow \mathcal{H}_{\{\mathfrak{a}\}}$, we have

$$
\left(\left(A, o_{\{\mathfrak{a}\}} A^{\prime}\right)\right)_{\{\mathfrak{a}\}}=\sum_{\epsilon= \pm} \sum_{s=0, \pm 1} \int_{\mathbb{R}^{3}} d^{3} \mathbf{x} f(\epsilon, s, \mathbf{x})^{*} \hat{O} f^{\prime}(\epsilon, s, \mathbf{x})=\sum_{\epsilon= \pm} \sum_{s=0, \pm 1} \int_{\mathbb{R}^{3}} d^{3} \mathbf{x}[\hat{O} f(\epsilon, s, \mathbf{x})]^{*} f^{\prime}(\epsilon, s, \mathbf{x}) .
$$

The above discussion shows that we may view the wave functions $f$ as elements of $\mathcal{H}^{\prime}$, and similarly to the case of KG fields, ${ }^{26,27}$ formulate the QM of Proca fields in terms of these wave functions. In this formulation the observables are Hermitian operators $\hat{O}$ acting on the wave functions. For example, the action of $\mathbf{x}_{0\{\mathfrak{a}\}}, \mathbf{p}_{0\{\mathfrak{a}\}}, s_{3\{\mathfrak{a}\}}$, and $s_{12\{\mathfrak{a}\}}$ on $A$ corresponds to the action of the operators $\hat{\mathbf{x}}_{0\{\mathfrak{a}\}}, \hat{\mathbf{p}}_{0\{\mathfrak{a}\}}, \hat{s}_{3\{\mathfrak{a}\}}$, and $\hat{s}_{12\{\mathfrak{a}\}}$ on $f$, where

$$
\begin{gathered}
\hat{\mathbf{x}}_{0\{\mathfrak{a}\}} f(\epsilon, s, \mathbf{x}):=\mathbf{x} f(\epsilon, s, \mathbf{x}), \quad \hat{\mathbf{p}}_{0\{\mathfrak{a}\}} f(\epsilon, s, \mathbf{x}):=-i \hbar \nabla f(\epsilon, s, \mathbf{x}), \\
\hat{s}_{3\{\mathfrak{a}\}} f(\epsilon, s, \mathbf{x}):=\epsilon f(\epsilon, s, \mathbf{x}), \quad \hat{s}_{12\{\mathfrak{a}\}} f(\epsilon, s, \mathbf{x}):=s f(\epsilon, s, \mathbf{x}) .
\end{gathered}
$$

These equations show that $f(\epsilon, s, \mathbf{x})$ are the position wave functions with definite chirality (sign of the energy) $\epsilon$ and spin $s$ (say along the $x^{3}$-direction). They are, however, not the eigenfunctions of the helicity operator. To see this, we recall from (40) that $\mathcal{H}^{\prime}=\tilde{\mathcal{H}} \oplus \tilde{\mathcal{H}}$, and the spin operator acting in $\widetilde{\mathcal{H}}$ is given by $\mathbf{S}$ of Eq. (25). This shows that the spin operator acting in $\mathcal{H}^{\prime}$ is (Throughout this paper we express spin and angular momentum operators in units of $\hbar$.)

$$
\mathbf{S}^{\prime}:=\left(\Sigma_{28},-\Sigma_{20}, \Sigma_{8}\right)=\left(\begin{array}{ll}
\mathbf{S} & 0 \\
0 & \mathbf{S}
\end{array}\right)
$$

Denoting the spin operator acting in $\mathcal{H}_{\{\mathfrak{a}\}}$ by $\mathbf{s}_{\{\mathfrak{a}\}}$, we have

$$
\mathbf{s}_{\{\mathfrak{a}\}}=\mathcal{U}_{\{\mathfrak{a}\}}^{-1} \mathbf{S}^{\prime} \mathcal{U}_{\{\mathfrak{a}\}} .
$$

The projection of this operator along the momentum $\mathbf{p}_{0\{\mathfrak{a}\}}$ gives the helicity operator,

$$
\left(\hat{\mathbf{p}}_{0\{\mathfrak{a}\}} \cdot \hat{\mathbf{s}}_{\{\mathfrak{a}\}}\right) f\left(\epsilon, s^{i}, \mathbf{x}\right)=\epsilon_{i j k} \partial_{j} f\left(\epsilon, s^{k}, \mathbf{x}\right),
$$

where $\left(s^{1}, s^{2}, s^{3}\right)=(+1,-1,0)$, and we employed $(147)$.

Similarly, the action of the Hamiltonian $\mathrm{h}$ on $A$ corresponds to the action of the operator $\hat{\mathrm{h}}:=\hat{s}_{3\{\mathfrak{a}\}} \sqrt{\hat{\mathbf{p}}_{0\{\mathfrak{a}\}}^{2}+m^{2} c^{2}}$ on the wave function $f$, 


$$
\hat{\mathrm{h}} f(\epsilon, s, \mathbf{x})=\hbar \epsilon \sqrt{-\nabla^{2}+\mathcal{M}^{2}} f(\epsilon, s, \mathbf{x}) .
$$

Subsequently, in view of (162) and (58), the dynamics of the evolving Proca field $A_{x^{0}}$ is determined in terms of the wave functions $f\left(\epsilon, s, \mathbf{x} ; x^{0}\right)=\left(\left(A_{\{\mathfrak{a}\} \mathbf{x}}^{(\epsilon, s)}, A_{x^{0}}\right)\right)_{\{\mathfrak{a}\}}$, according to

$$
i \hbar \partial_{0} f\left(\epsilon, s, \mathbf{x} ; x^{0}\right)=\epsilon \sqrt{-\hbar^{2} \nabla^{2}+m^{2} c^{2}} f\left(\epsilon, s, \mathbf{x} ; x^{0}\right) .
$$

Furthermore, applying $i \partial_{0}$ to both sides of (163), we can check that the wave functions $f$ also satisfy the KG equation: $\left[\partial_{0}^{2}-\nabla^{2}+\mathcal{M}^{2}\right] f\left(\epsilon, s, \mathbf{x} ; x^{0}\right)=0$.

Next, recall that because the time-reversal operator (79) acting in $\mathcal{H}^{\prime}$ commutes with $\mathbf{X}_{0}^{\prime}$, the eigenvectors $\xi_{\mathbf{x}}^{(\epsilon, s)}$ may be taken to be real. In this case the action of the time-reversal operator $T=$ PT on any $A \in \mathcal{H}_{\{\mathfrak{a}\}}$ is equivalent to the complex-conjugation of the associated wave function $f$, i.e., $\hat{T} f(\epsilon, s, \mathbf{x})=f(\epsilon, s, \mathbf{x})^{*}$. Similarly, we can identify the operators $\hat{s}_{3}$ and $\hat{s}_{12}$, respectively, with the chirality and spin operators acting on the wave functions $f$.

As we shall see in Sec. VII the wave functions $f(\epsilon, s, \mathbf{x})$ furnish a position representation for the QM of the Proca fields. The corresponding position operator is the spin-1 analog of the Newton-Wigner position operator for the KG field ${ }^{21,22}$ and similarly to the latter fails to be relativistically covariant. (See, however, Ref. 48.) This means that the above-mentioned positionrepresentation provides a noncovariant description of a quantum system that also admits a unitaryequivalent covariant description in terms of the Hilbert space $\mathcal{H}_{\{\mathfrak{a}\}}$ and the Hamiltonian h.

\section{POSITION, SPIN, AND LOCALIZED STATES}

As discussed in Ref. 27 for the KG fields, the canonical quantization scheme that provides the physical meaning of the observables yields the Foldy representation of the quantum system. (In the classical limit, each component of a Proca field corresponds to a classical free particle of energy $E= \pm \sqrt{p^{2}+m^{2} c^{2}}$. Upon quantization $E \rightarrow \pm \hbar \sqrt{-\nabla^{2}+\mathcal{M}^{2}}$ which signifies the relevance of the Foldy representation.) This suggests that the operators $\hat{\mathbf{x}}_{0\{\mathfrak{a}\}}$ and $\hat{\mathbf{p}}_{0\{\mathfrak{a}\}}$ that clearly satisfy the canonical commutation relations may be identified with the position and momentum operators acting on the wave functions $f$. This in turn means that the operators $\mathbf{X}_{0}^{\prime}$ and $\mathbf{P}_{0}^{\prime}$ in the Foldy representation and the operators $\mathbf{x}_{0\{\mathfrak{a}\}}$ and $\mathbf{p}_{0\{\mathfrak{a}\}}$ in the $\left(\mathcal{H}_{\{\mathfrak{a}\}}, \mathrm{h}\right)$-representation also describe the position and momentum observables. In particular, the basis vectors $\xi_{\mathbf{x}}^{(\epsilon, s)}$ and $A_{\{\mathfrak{a}\} \mathbf{x}}^{(\epsilon, s)}$ determine the states of the system with a definite position value $\mathbf{x}$; they are localized in space. They also have definite charge or chirality (sign of the energy) and spin (say along the $x^{3}$-direction).

By construction, $A_{\{\mathfrak{a}\} \mathbf{x}}^{(\epsilon, s)}$ are delta-function normalized position eigenvectors, i.e., they satisfy (150) and

$$
\mathbf{x}_{0\{\mathfrak{a}\}} A_{\{\mathfrak{a}\} \mathbf{x}}^{(\epsilon, s)}=\mathbf{x} A_{\{\mathfrak{a}\} \mathbf{x}}^{(\epsilon, s)} .
$$

Similarly, we identify the chirality operator and the spin operator along the $x^{3}$-direction with $\mathrm{C}$ $=\mathcal{U}_{\{\mathfrak{a}\}}^{-1} \Sigma_{3} \mathcal{U}_{\{\mathfrak{a}\}}$ and $s_{12}=\mathcal{U}_{\{a\}}^{-1} \Sigma_{12} \mathcal{U}_{\{\mathfrak{a}\}}$, respectively. This implies

$$
\mathrm{C} A_{\{\mathfrak{a}\} \mathbf{x}}^{(\epsilon, s)}=\epsilon A_{\{\mathfrak{a}\} \mathbf{x}}^{(\epsilon, s)}, \quad s_{12} A_{\{\mathfrak{a}\} \mathbf{x}}^{(\epsilon, s)}=s A_{\{\mathfrak{a}\} \mathbf{x}}^{(\epsilon, s)} .
$$

In view of Eqs. (150), (164), and (165), the state vectors $A_{\{a\} \mathbf{x}}^{(\epsilon, s)}$ represent spatially localized Proca fields with definite chirality $\epsilon$ and spin $s$. We can associate each Proca field $A \in \mathcal{H}_{\{\mathfrak{a}\}}$ with a unique position wave function, namely, $f(\epsilon, s, \mathbf{x})$. As we explained in Sec. VI, we can use these wave functions to represent all the physical quantities associated with the Proca fields. We also emphasize that according to (161), $f(\epsilon, s, \mathbf{x})$ are not the helicity eigenfunctions. Therefore, the state vectors $A_{\{a\} \mathbf{x}}^{(\epsilon, s)}$ do not have definite helicity. This is in complete accordance with the Heisenberg uncertainty principle: since $A_{\{a\} \mathbf{x}}^{(\epsilon, s)}$ are localized states (with definite position) they do not have definite momentum and hence definite helicity. 
Next, we recall that two Hilbert spaces $\mathcal{H}_{\{\mathfrak{a}\}}$ for different choices of the parameters $\{\mathfrak{a}\}$ are unitary equivalent. We can use (148) and (149), and the unitary operator (117) to obtain the explicit form of the localized Proca fields and the physical observables acting in $\mathcal{H}_{\{\mathfrak{a}\}}$. The resulting expressions for the position and spin operators are highly complicated. Therefore, in the following we shall only derive the explicit form of these operators in the covariant representation $(\mathcal{H}, \mathrm{h})$.

\section{A. Explicit form of the localized states}

As suggested by (155), the wave functions $f(\epsilon, s, \mathbf{x})$ belong to $L^{2}\left(\mathbb{R}^{3}\right)$. Moreover due to the implicit dependence of $A_{\mathbf{x}}^{(\epsilon, s)}$ on the $x_{0}^{0}$ appearing in the expression for $\mathcal{U}, f(\epsilon, s, \mathbf{x})$ depend on $x_{0}^{0}$. As in the case of KG fields this dependence becomes explicit, if we express $f(\epsilon, s, \mathbf{x})$ in terms of $A$ directly. To see this, we first substitute (67), (68), and (142) in (149) to obtain

$$
A_{\mathbf{x}}^{0\left(\epsilon, s^{i}\right)}\left(x^{0}\right)=\epsilon \frac{D^{-1 / 4}}{\sqrt{\mathcal{M} \kappa}} e^{-i \epsilon\left(x^{0}-x_{0}^{0}\right) D^{1 / 2}} \mathfrak{K}^{i}|\mathbf{x}\rangle, \quad \mathbf{A}_{\mathbf{x}}^{\left(\epsilon, s^{i}\right)}\left(x^{0}\right)=\sqrt{\frac{\mathcal{M}}{\kappa}} \mathfrak{U}^{-1} e^{-i \epsilon\left(x^{0}-x_{0}^{0}\right) D^{1 / 2}}|\mathbf{x}\rangle \otimes e_{s^{i}},
$$

where $\left(s^{1}, s^{2}, s^{3}\right)=(+1,-1,0)$. We then use this equation and (55) to compute the right-hand side of (151). This yields

$$
f\left(\epsilon, s^{i}, \mathbf{x}\right)=\sqrt{\frac{\kappa}{\mathcal{M}}} e^{i \epsilon\left(x^{0}-x_{0}^{0}\right) \hat{D}^{1 / 2}}\left[\mathfrak{U A}_{\epsilon}\left(x^{0}, \mathbf{x}\right)\right]^{i},
$$

where $A_{\epsilon}^{\mu}$ is the definite-chirality (definite-energy) component of $A^{\mu}$ with chirality $\epsilon$. Because $A_{\epsilon}^{\mu}$ and consequently $\mathfrak{U A}_{\epsilon}$ satisfy the Foldy equation (126), we have $e^{i \epsilon\left(x^{0}-x_{0}^{0}\right) D^{1 / 2}} \mathfrak{U} \mathbf{A}_{\boldsymbol{\epsilon}}\left(x^{0}, \mathbf{x}\right)$ $=\mathfrak{U} \mathbf{A}_{\epsilon}\left(x_{0}^{0}, \mathbf{x}\right)$. Inserting this in (167), we find the following manifestly $x_{0}^{0}$-dependent expression for $f\left(\epsilon, s^{i}, \mathbf{x}\right)$ :

$$
f\left(\epsilon, s^{i}, \mathbf{x}\right)=\sqrt{\frac{\kappa}{\mathcal{M}}}\left[\mathfrak{U} \mathbf{A}_{\epsilon}\left(x_{0}^{0}, \mathbf{x}\right)\right]^{i} .
$$

Next, we use (166) to compute the value of the localized Proca fields $A_{\mathbf{y}}^{\left(\epsilon, s^{i}\right)}$ at a space-time point $\left(x^{0}, \mathbf{x}\right)$,

$$
A_{\mathbf{y}}^{\left(\epsilon, s^{i}\right)}\left(x^{0}, \mathbf{x}\right):=\left\langle\mathbf{x} \mid A_{\mathbf{y}}^{\left(\epsilon, s^{i}\right)}\left(x^{0}\right)\right\rangle .
$$

Doing the necessary calculations, we obtain

$$
\begin{aligned}
A_{\mathbf{y}}^{0(\epsilon,+1)}(x)=i \epsilon \sin \theta \cos \phi I_{1}, & A_{\mathbf{y}}^{0(\epsilon,-1)}(x)=i \epsilon \sin \theta \sin \phi I_{1}, \quad A_{\mathbf{y}}^{0(\epsilon, 0)}(x)=i \epsilon \cos \theta I_{1}, \\
& \mathbf{A}_{\mathbf{y}}^{(\epsilon,+1)}(x)=\left(\begin{array}{c}
v_{1} \\
v_{2} \\
v_{3}
\end{array}\right), \quad \mathbf{A}_{\mathbf{y}}^{(\epsilon,-1)}(x)=\left(\begin{array}{c}
v_{2} \\
v_{4} \\
v_{5}
\end{array}\right), \quad \mathbf{A}_{\mathbf{y}}^{(\epsilon, 0)}(x)=\left(\begin{array}{c}
v_{3} \\
v_{5} \\
v_{6}
\end{array}\right),
\end{aligned}
$$

where $x:=\left(x^{0}, \mathbf{x}\right), \theta$ and $\phi$ are the polar and azimuthal angles representing the direction of $\mathbf{x}-\mathbf{y}$,

$$
v_{1}:=I_{2}+\sin ^{2} \theta \cos ^{2} \phi I_{3}, \quad v_{2}:=\frac{1}{2} \sin ^{2} \theta \sin (2 \phi) I_{3}, \quad v_{3}:=\frac{1}{2} \sin (2 \theta) \cos \phi I_{3},
$$




$$
v_{4}:=I_{2}+\sin ^{2} \theta \sin ^{2} \phi I_{3}, \quad v_{5}:=\frac{1}{2} \sin (2 \theta) \sin \phi I_{3}, \quad v_{6}:=I_{2}+\cos ^{2} \theta I_{3},
$$

and

$$
\begin{gathered}
I_{1}:=\frac{|\mathbf{x}-\mathbf{y}|}{2 \pi^{2} \sqrt{\mathcal{M} \kappa}} \int_{0}^{\infty} d k k^{2} \Omega_{1}(\epsilon, k) \Omega_{2}(k), \\
I_{2}:=\sqrt{\frac{\mathcal{M}}{\kappa}} \frac{1}{2 \pi^{2}} \int_{0}^{\infty} d k \Omega_{1}(\epsilon, k)\left\{\frac{k \sin (k|\mathbf{x}-\mathbf{y}|)}{|\mathbf{x}-\mathbf{y}|}+\Omega_{2}(k)\left\{\mathcal{M}^{-1}\left[k^{2}+\mathcal{M}^{2}\right]^{1 / 2}-1\right\}\right\}, \\
I_{3}:=\sqrt{\frac{\mathcal{M}}{\kappa}} \frac{1}{2 \pi^{2}} \int_{0}^{\infty} d k \Omega_{1}(\epsilon, k)\left\{\frac{k \sin (k|\mathbf{x}-\mathbf{y}|)}{|\mathbf{x}-\mathbf{y}|}-3 \Omega_{2}(k)\right\}\left\{\mathcal{M}^{-1}\left[k^{2}+\mathcal{M}^{2}\right]^{1 / 2}-1\right\}, \\
\Omega_{1}(\epsilon, k):=\frac{\exp \left[-i \epsilon\left(x^{0}-x_{0}^{0}\right) \sqrt{k^{2}+\mathcal{M}^{2}}\right]}{\left[k^{2}+\mathcal{M}^{2}\right]^{1 / 4}}, \quad \Omega_{2}(k):=\frac{\sin (k|\mathbf{x}-\mathbf{y}|)}{k|\mathbf{x}-\mathbf{y}|^{3}}-\frac{\cos (k|\mathbf{x}-\mathbf{y}|)}{|\mathbf{x}-\mathbf{y}|^{2}} .
\end{gathered}
$$

For $x^{0}=x_{0}^{0}$, the integrals on the right-hand side (172)-(174) can be expressed in terms of the Bessel K-function $\left(K_{n}\right)$ and the hypergeometric function $\left({ }_{p} F_{q}\right)$. The result, for both $\epsilon=-1$ and 1 , is

$$
\begin{aligned}
& I_{1}=\sqrt{\frac{\mathcal{M}}{\kappa}}\left[2^{3 / 4} \pi^{3 / 2} \Gamma\left(\frac{1}{4}\right)\right]^{-1}\left(\frac{\mathcal{M}}{z}\right)^{5 / 4}\left\{\frac{5}{2 \mathcal{M} z} K_{5 / 4}(\mathcal{M} z)+K_{1 / 4}(\mathcal{M} z)\right\}, \\
& I_{2}=\sqrt{\frac{\mathcal{M}}{\kappa}}\left[2^{3 / 4} \pi^{3 / 2} \Gamma\left(\frac{1}{4}\right)\right]^{-1}\left(\frac{\mathcal{M}}{z}\right)^{5 / 4}\left\{K_{5 / 4}(\mathcal{M} z)+\frac{1}{\mathcal{M} z} K_{1 / 4}(\mathcal{M} z)+\frac{\Gamma\left(\frac{1}{4}\right)^{2}}{4 \pi(\mathcal{M} z)^{3 / 2}} K_{3 / 4}(\mathcal{M} z)\right. \\
& +\frac{1}{(\mathcal{M} z)^{3 / 4}}\left(\frac{2 \pi}{\Gamma\left(\frac{1}{4}\right)}{ }_{1} F_{2}\left[\frac{1}{2} ; \frac{5}{4}, \frac{3}{2} ; \frac{\mathcal{M}^{2} z^{2}}{4}\right]+\frac{\Gamma\left(\frac{1}{4}\right)^{3}}{3 \pi \cdot 2^{7 / 4} 1} F_{2}\left[\frac{1}{2} ; \frac{3}{2}, \frac{7}{4} ; \frac{\mathcal{M}^{2} z^{2}}{4}\right]\right) \\
& \left.+\frac{\Gamma\left(\frac{1}{4}\right)}{(\mathcal{M} z)^{5 / 4}}\left(\frac{1}{2^{3 / 4} \mathcal{M} z}{ }_{1} F_{2}\left[\frac{1}{4} ; \frac{1}{4}, \frac{3}{4} ; \frac{\mathcal{M}^{2} z^{2}}{4}\right]-2^{1 / 4}{ }_{1} F_{2}\left[\frac{1}{4} ; \frac{3}{4}, \frac{5}{4} ; \frac{\mathcal{M}^{2} z^{2}}{4}\right]\right)\right\}, \\
& I_{3}=\sqrt{\frac{\mathcal{M}}{\kappa}}\left[2^{3 / 4} \pi^{3 / 2} \Gamma\left(\frac{1}{4}\right)\right]^{-1}\left(\frac{\mathcal{M}}{z}\right)^{5 / 4}\left\{\frac{3 K_{1 / 4}(\mathcal{M} z)}{\mathcal{M} z}+\frac{K_{5 / 4}(\mathcal{M} z)}{(\mathcal{M} z)^{5 / 4}}+\frac{\Gamma\left(\frac{1}{4}\right)^{2}}{4 \pi(\mathcal{M} z)^{1 / 2}}\left[K_{7 / 4}(\mathcal{M} z)\right.\right. \\
& \left.+\frac{3 K_{3 / 4}(\mathcal{M} z)}{\mathcal{M} z}\right]+\frac{1}{(2 \mathcal{M} z)^{3 / 4}}\left(\frac{12 \pi}{\Gamma\left(\frac{1}{4}\right)}{ }_{1} F_{2}\left[\frac{1}{2} ; \frac{5}{4}, \frac{3}{2} ; \frac{\mathcal{M}^{2} z^{2}}{4}\right]+\frac{\Gamma\left(\frac{1}{4}\right)^{3}}{2 \pi}{ }_{1} F_{2}\left[\frac{1}{2} ; \frac{3}{2}, \frac{7}{4} ; \frac{\mathcal{M}^{2} z^{2}}{4}\right]\right) \\
& \left.+\frac{3 \Gamma\left(\frac{1}{4}\right)}{(\mathcal{M} z)^{5 / 4}}\left(\frac{1}{2^{3 / 4} \mathcal{M} z}{ }_{1} F_{2}\left[-\frac{1}{4} ; \frac{1}{4}, \frac{3}{4} ; \frac{\mathcal{M}^{2} z^{2}}{4}\right]+2^{1 / 4}{ }_{1} F_{2}\left[\frac{1}{4} ; \frac{3}{4}, \frac{5}{4} ; \frac{\mathcal{M}^{2} z^{2}}{4}\right]\right)\right\},
\end{aligned}
$$

where $z:=|\mathbf{x}-\mathbf{y}|$ and $\Gamma$ is the Gamma function. Figure 1 gives the graphs of $I_{1}, I_{2}$, and $I_{3}$. They involve a $\delta$-function-like singularity at $|\mathbf{x}-\mathbf{y}|=0$. This is a manifestation of the fact that $A_{\mathbf{y}}^{(\epsilon, s)}$ are localized at $\mathbf{y}$.

As seen from Eqs. (170) and (171), the localized Proca fields, unlike the localized KG fields, ${ }^{6}$ depend on the angles $\theta$ and $\phi$ (direction of $\mathbf{x}-\mathbf{y}$ ). But as for the KG fields their position wave functions involve the delta functions; the position wave function $f_{(\epsilon, s, \mathbf{x})}\left(\epsilon^{\prime}, s^{\prime}, \mathbf{x}^{\prime}\right)$ for $A_{\mathbf{x}}^{(\epsilon, s)}\left(x_{0}^{0}\right)$ has the form $\delta_{\epsilon, \epsilon^{\prime}} \delta_{s, s^{\prime}} \delta^{3}\left(\mathbf{x}-\mathbf{x}^{\prime}\right)$. 


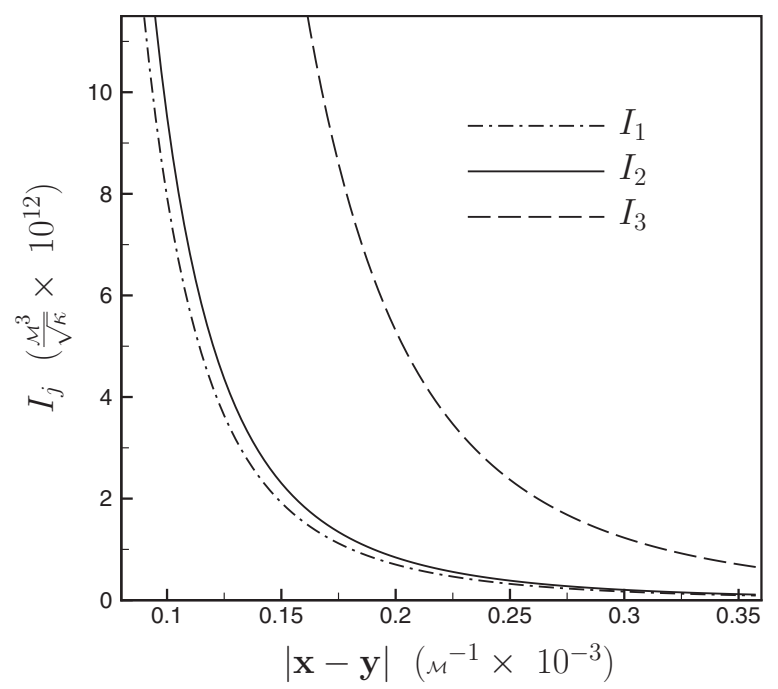

FIG. 1. Plots of $I_{1}, I_{2}$, and $I_{3}$ in terms of the radial distance $|\mathbf{x}-\mathbf{y}|$. The radial distance is scaled with the Compton wave length $\mathcal{M}^{-1}$.

Finally, we wish to emphasize that to the best of our knowledge the explicit form of the localized Proca fields $A_{\mathrm{x}}^{(\epsilon, s)}$ has not been previously given. It is remarkable that we have obtained these localized states without pursuing the axiomatic approach of Ref. 21. The latter gives rise to the Bargmann-Wigner localized fields. ${ }^{21,20}$

\section{B. Position, spin, and helicity operators}

Using the unitary map $\mathcal{U}$ and its inverse, we can obtain the explicit form of the position operator $\mathbf{x}_{0}$ that is defined to act on the Proca fields $A \in \mathcal{H}$. Note that $\chi:=\mathbf{x}_{0} A$ is a three-component field whose components satisfy both (11) and (13). Therefore it is uniquely determined in terms of the initial data $\left(\chi\left(x_{0}^{0}\right), \dot{\chi}\left(x_{0}^{0}\right)\right)$ for some $x_{0}^{0} \in \mathbb{R}$. One can compute the latter using (148), (65), (67), and (68), and the identities: $D=\mathfrak{K}^{2}+\mathcal{M}^{2}$,

$$
\begin{gathered}
\mathfrak{h} \mathbf{S} \mathfrak{h}=\frac{\mathfrak{K}}{|\mathfrak{K}|} \mathfrak{h}, \\
{[\mathbf{x}, \mathfrak{h}]=\frac{i}{|\mathfrak{K}|} \mathbf{S}-\frac{i \mathfrak{K}}{|\mathfrak{K}|^{2}} \mathfrak{h}, \quad\left[\mathbf{x}, \mathfrak{h}^{2}\right]=\frac{i}{|\mathfrak{K}|}(\mathfrak{h} \mathbf{S}+\mathbf{S} \mathfrak{h})-\frac{2 i \mathfrak{K}}{|\mathfrak{K}|^{2}} \mathfrak{h}^{2},}
\end{gathered}
$$

and $[F(\mathfrak{K}), \mathbf{x}]=-i \nabla_{\mathfrak{K}} F(\mathfrak{K})$, where $F$ is a differentiable function. This yields

$$
\begin{gathered}
\chi^{0}\left(x_{0}^{0}\right)=\mathfrak{Y} A^{0}\left(x_{0}^{0}\right)+\frac{1}{\mathcal{M} D^{1 / 2}} \dot{\mathbf{A}}\left(x_{0}^{0}\right), \quad \dot{\chi}^{0}\left(x_{0}^{0}\right)=\left(\mathfrak{Y}-\frac{i \mathfrak{K}}{D}\right) \dot{A}^{0}\left(x_{0}^{0}\right)-\frac{D^{1 / 2}}{\mathcal{M}} \mathbf{A}\left(x_{0}^{0}\right), \\
\boldsymbol{\chi}\left(x_{0}^{0}\right)=\mathfrak{X} \mathbf{A}\left(x_{0}^{0}\right), \quad \dot{\boldsymbol{\chi}}\left(x_{0}^{0}\right)=\left(\mathfrak{X}-\frac{i \mathfrak{K}}{D}\right) \dot{\mathbf{A}}\left(x_{0}^{0}\right),
\end{gathered}
$$

where

$$
\mathfrak{Y}:=\mathbf{x}+\frac{i \mathfrak{K}}{2 D}+\frac{i \mathfrak{K}}{\mathcal{M}\left(D^{1 / 2}+\mathcal{M}\right)},
$$




$$
\begin{aligned}
\mathfrak{X}:= & \mathbf{x}-\frac{i}{2} \mathfrak{K} D^{-1}+i \mathfrak{K}\left[D^{-1}-\mathcal{M}^{-1} D^{-1 / 2}\right] \mathfrak{h}^{2}+\frac{i}{|\mathfrak{K}|}\left[1-\mathcal{M} D^{-1 / 2}\right] \mathfrak{h} \mathbf{S}+\frac{i}{|\mathfrak{K}|}\left[\mathcal{M}^{-1} D^{1 / 2}-1\right] \mathbf{S h}, \\
& =\mathbf{x}-\frac{i \mathfrak{K}}{2 D}-\frac{i \mathfrak{K}(\mathfrak{K} \cdot \mathbf{S})^{2}}{\mathcal{M} D\left(D^{1 / 2}+\mathcal{M}\right)}+\frac{i[\mathbf{S}(\mathfrak{K} \cdot \mathbf{S})+(\mathfrak{K} \cdot \mathbf{S}) \mathbf{S}]}{2 \mathcal{M} D^{1 / 2}}-\frac{\left(D^{1 / 2}-\mathcal{M}\right)(\mathfrak{K} \times \mathbf{S})}{2 \mathcal{M} D^{1 / 2}\left(D^{1 / 2}+\mathcal{M}\right)} .
\end{aligned}
$$

Next, we employ (17) to express $\chi\left(x^{0}\right)$ in terms of (181) and (182). Simplify the resulting expression, we find, for all $x^{0} \in \mathbb{R}$,

$$
\begin{gathered}
\chi^{0}\left(x^{0}\right)=\left\{\mathfrak{Y}-i\left(x^{0}-x_{0}^{0}\right) \mathfrak{K} D^{-1} \partial_{0}\right\} A^{0}\left(x^{0}\right)+\frac{1}{\mathcal{M} D^{1 / 2}} \dot{\mathbf{A}}\left(x^{0}\right), \\
\boldsymbol{\chi}\left(x^{0}\right)=\left\{\mathfrak{X}-i\left(x^{0}-x_{0}^{0}\right) \mathfrak{K} D^{-1} \partial_{0}\right\} \mathbf{A}\left(x^{0}\right) .
\end{gathered}
$$

In addition to being a Hermitian operator acting in the physical Hilbert space $\mathcal{H}$, the position operator $\mathbf{x}_{0}$ has the following notable properties.

(1) It respects the charge superselection rule, ${ }^{49}$ for it commutes with the chirality operator $\mathrm{C}$ $=s_{3}$. This is easily seen by noting that $\mathbf{x}_{0}$ and $s_{3}$ are, respectively, obtained via a similarity transformation (148) from $\mathbf{X}_{0}^{\prime}$ and $\mathcal{S}_{3}^{\prime}=\Sigma_{3}$, and that according to (141), $\left[\mathbf{X}_{0}^{\prime}, \Sigma_{3}\right]=0$.

(2) It has commuting components, and it commutes with the spin operator. This arises because it is obtained via a similarity transformation (148) from $\mathbf{X}_{0}^{\prime}$ which has commuting components and commutes with $\mathbf{S}^{\prime}$.

(3) Its $x^{0}$-derivative gives the relativistic velocity operator; i.e., we have

$$
\mathbf{v}:=\frac{d \mathbf{x}_{0}}{d x^{0}}=i\left[\mathrm{~h}, \mathbf{x}_{0}\right]=i \mathcal{U}^{-1}\left[H^{\prime}, \mathbf{X}^{\prime}\right] \mathcal{U}=\frac{\mathbf{p}_{0}}{\sqrt{\mathbf{p}_{0}^{2}+m^{2} c^{2}}} \mathrm{C} .
$$

As we noticed in Ref. 28, this result means that the physical momentum is $\mathbf{p}_{0} \mathrm{C}$.

(4) It has the correct nonrelativistic limit: As $c \rightarrow \infty, \mathbf{x}_{0} \rightarrow \mathbf{x}$.

Similarly, we can obtain the explicit form of the spin operator $\mathbf{s}_{0}$ acting on $A \in \mathcal{H}$. Again, noting that $\mathscr{S}:=\mathbf{s}_{0} A$ is a three-component field whose components satisfy (11) and (13), we can determine $\mathscr{S}$ in terms of the initial data $\left(\mathscr{S}\left(x_{0}^{0}\right), \dot{\mathscr{S}}\left(x_{0}^{0}\right)\right)$. Using (65), (67), (68), (148), and (179), and

$$
[\mathbf{S}, \mathfrak{h}]=\frac{i}{|\mathfrak{K}|}(\mathfrak{K} \times \mathbf{S}), \quad\left[\mathbf{S}, \mathfrak{h}^{2}\right]=\frac{i}{|\mathfrak{K}|}[(\mathfrak{K} \times \mathbf{S}) \mathfrak{h}+\mathfrak{h}(\mathfrak{K} \times \mathbf{S})],
$$

these initial values are given by

$$
\begin{gathered}
\mathscr{S}^{0}\left(x_{0}^{0}\right)=\mathcal{M}^{-1} D^{-1 / 2} \mathfrak{K} \times \dot{\mathbf{A}}\left(x_{0}^{0}\right), \quad \dot{\mathscr{S}}^{0}\left(x_{0}^{0}\right)=-\mathcal{M}^{-1} D^{1 / 2} \mathfrak{K} \times \mathbf{A}\left(x_{0}^{0}\right), \\
\dot{\mathscr{S}}\left(x_{0}^{0}\right)=\widetilde{\mathbf{s}}_{0} \mathbf{A}\left(x_{0}^{0}\right), \quad \ddot{\mathscr{S}}\left(x_{0}^{0}\right)=\widetilde{\mathbf{s}}_{0} \dot{\mathbf{A}}\left(x_{0}^{0}\right),
\end{gathered}
$$

where

$$
\begin{aligned}
\widetilde{\mathbf{s}}_{0} & =\mathbf{S}+\frac{i}{|\mathfrak{K}|}\left[1-\mathcal{M} D^{-1 / 2}\right] \mathfrak{h}(\mathfrak{K} \times \mathbf{S})+\frac{i}{|\mathfrak{K}|}\left[\mathcal{M}^{-1} D^{1 / 2}-1\right](\mathfrak{K} \times \mathbf{S}) \mathfrak{h} \\
& =\frac{D+\mathcal{M}^{2}}{2 \mathcal{M} D^{1 / 2}} \mathbf{S}-\frac{\left(D^{1 / 2}-\mathcal{M}\right) \mathfrak{K}(\mathfrak{K} \cdot \mathbf{S})}{2 \mathcal{M} D^{1 / 2}\left(D^{1 / 2}+\mathcal{M}\right)}+\frac{i\{(\mathfrak{K} \cdot \mathbf{S})(\mathfrak{K} \times \mathbf{S})+(\mathfrak{K} \times \mathbf{S})(\mathfrak{K} \cdot \mathbf{S})\}}{2 \mathcal{M} D^{1 / 2}} .
\end{aligned}
$$

Next, we use (17) to express $\mathscr{S}\left(x^{0}\right)$ in terms of the initial data (188). This leads to 


$$
\mathscr{S}^{0}\left(x^{0}\right)=\mathcal{M}^{-1} D^{-1 / 2} \mathfrak{K} \times \dot{\mathbf{A}}\left(x^{0}\right), \quad \dot{\mathscr{S}}\left(x^{0}\right)=\widetilde{\mathbf{s}}_{0} \mathbf{A}\left(x^{0}\right) .
$$

As seen from (185), (186), and (190), the action of the position and spin operators on a Proca field $A$ mixes the components of the field. It is clearly more complicated than the action of the corresponding operators for the KG fields. ${ }^{6}$

Next, we evaluate the action of the momentum, angular momentum, and helicity operators on $A$. Because $\mathbf{P}_{0}^{\prime}$ and $\rho$ commute, in view of (148) and (59), we have $\mathbf{p}_{0}=U_{x_{0}^{0}}^{-1} \mathbf{P}_{0}^{\prime} U_{x_{0}^{0}}$. This in turn implies $\left[\mathbf{p}_{0} A\right]\left(x^{0}\right)=\mathbf{p} A\left(x^{0}\right)$ for all $x^{0} \in \mathbb{R}$. Furthermore, using (185), (186), and (190), we can show that the angular momentum operator (in units of $\hbar$ ) $\mathbf{L}:=\hbar^{-1} \mathbf{x}_{0} \times \mathbf{p}_{0}$ acts on $A$ according to

$$
\begin{aligned}
& \mathcal{L}^{0}\left(x^{0}\right)=\hbar^{-1}(\mathbf{x} \times \mathbf{p}) A^{0}\left(x^{0}\right)-\mathscr{S}^{0}\left(x^{0}\right), \\
& \overrightarrow{\mathcal{L}}\left(x^{0}\right)=\left\{\hbar^{-1} \mathbf{x} \times \mathbf{p}+\mathbf{S}\right\} A\left(x^{0}\right)-\dot{\mathscr{S}}\left(x^{0}\right), \quad \forall x^{0} \in \mathrm{R},
\end{aligned}
$$

where $\mathcal{L}\left(x^{0}\right):=[\mathbf{L} A]\left(x^{0}\right)$, and $\mathscr{S}\left(x^{0}\right):=\left[\mathbf{s}_{0} A\right]\left(x^{0}\right)$ is given in (190). Therefore, unlike the position $\mathbf{x}_{0}$ and spin $\mathbf{s}_{0}$ operators, the (linear) momentum $\mathbf{p}_{0}$ and the total angular momentum $\mathbf{M}:=\mathbf{L}+\mathbf{s}_{0}$ operators have the same expressions as in nonrelativistic QM. (Note that the corresponding particle in nonrelativistic $\mathrm{QM}$ is described by $\mathbf{A},{ }^{46}$ i.e., we should set $A^{0}=0$.) Similarly, we can compute the action of the helicity operator on $A$. In view of $(190)$, we find $\left[\left(\mathbf{p}_{0} \cdot \mathbf{s}_{0}\right) A\right]\left(x^{0}\right)$ $=\left(0,(\mathbf{p} \cdot \mathbf{S}) \mathbf{A}\left(x^{0}\right)\right)$, which is in complete accordance with our previous result $(100)$.

Again, to the best of our knowledge, the actions of the position, spin, and other observables on a (covariant) Proca field $A$ have not been previously given. Earlier works on the subject ${ }^{19-22}$ calculate the position and spin operators that act either on the six-component fields, i.e., in the Hilbert space $\mathcal{K}$, or on the Bargmann-Wigner's second-rank 4-spinors. One can use the unitary transformation $\rho: \mathcal{K} \rightarrow \mathcal{H}^{\prime}$ to compute the position $\left(\mathbf{X}_{0}:=\rho^{-1} \mathbf{X}_{0}^{\prime} \rho\right)$ and $\operatorname{spin}\left(\mathbf{S}_{0}:=\rho^{-1} \mathbf{S}^{\prime} \rho\right)$ operators acting in $\mathcal{K}$. The result is

$$
\begin{aligned}
& \mathbf{X}_{0}=\mathbf{x}-\frac{D^{1 / 2}-\mathcal{M}}{2 \mathcal{M} D^{1 / 2}\left(D^{1 / 2}+\mathcal{M}\right)}(\mathfrak{K} \times \mathbf{S})+\sigma_{1}\left\{-\frac{i \mathfrak{K}}{2 D}-\frac{i \mathfrak{K}(\mathfrak{K} \cdot \mathbf{S})^{2}}{\mathcal{M} D\left(D^{1 / 2}+\mathcal{M}\right)}+\frac{i[\mathbf{S}(\mathfrak{K} \cdot \mathbf{S})+(\mathfrak{K} \cdot \mathbf{S}) \mathbf{S}]}{2 \mathcal{M} D^{1 / 2}}\right\}, \\
& \mathbf{S}_{0}=\frac{D+\mathcal{M}^{2}}{2 \mathcal{M} D^{1 / 2}} \mathbf{S}-\frac{D^{1 / 2}-\mathcal{M}}{2 \mathcal{M} D^{1 / 2}\left(D^{1 / 2}+\mathcal{M}\right)} \mathfrak{K}(\mathfrak{K} \cdot \mathbf{S})+\frac{i}{2 \mathcal{M} D^{1 / 2}} \sigma_{1}\{(\mathfrak{K} \cdot \mathbf{S})(\mathfrak{K} \times \mathbf{S})+(\mathfrak{K} \times \mathbf{S})(\mathfrak{K} \cdot \mathbf{S})\} .
\end{aligned}
$$

These are exactly the position and spin operators that are obtained by Case in Ref. 16.

Following the treatment of the KG fields in Refs. 27 and 28, we can identify the coherent states of Proca fields with the eigenstates of the annihilation operator $\mathbf{a}:=\sqrt{k / 2 \hbar}\left(\mathbf{x}_{0}+i k^{-1} \mathbf{p}_{0}\right)$, where $k \in R$. Because both $\mathbf{x}_{0}$ and $\mathbf{p}_{0}$ commute with the chirality $\left(s_{3}\right)$ and spin $\left(s_{12}\right)$ operators, so does a. Hence, we can introduce a set of coherent states with definite chirality and spin. The corresponding state vectors $\mid \mathbf{z}, \epsilon, s)$ are defined as the common eigenvectors of $\mathbf{a}, s_{3}$, and $s_{12}$, i.e., $\mathbf{a} \mid \mathbf{z}, \boldsymbol{\epsilon}, s)=\mathbf{z} \mid \mathbf{z}, \boldsymbol{\epsilon}, s)$ and $\left.\left.\left.\left.s_{3} \mid \mathbf{z}, \boldsymbol{\epsilon}, s\right)=\epsilon \mid \mathbf{z}, \boldsymbol{\epsilon}, s\right), s_{12} \mid \mathbf{z}, \epsilon, s\right)=s \mid \mathbf{z}, \boldsymbol{\epsilon}, s\right)$, where $\mathbf{z} \in \mathbb{C}^{3}, \boldsymbol{\epsilon} \in\{-,+\}$, and $s$ $\in\{-1,0,+1\}$. We studied these coherent states and found that they have essentially the same properties as the coherent states of the KG fields that we explored in Ref. 28.

\section{PROBABILITY DENSITY FOR SPATIAL LOCALIZATION OF A FIELD}

We may employ the procedure outlined in Ref. 6 to find the probability density for the spatial localization of a Proca field. As in nonrelativistic QM, we identify the probability of the localization of a Proca field $A$ in a region $V \subseteq \mathbb{R}^{3}$, at time $t_{0}=x_{0}^{0} / c$, with 


$$
P_{V}=\int_{V} d^{3} \mathbf{x}\left\|\Pi_{\mathbf{x}} A\right\|^{2}
$$

where $\Pi_{\mathbf{x}}$ is the projection operator onto the eigenspace of $\mathbf{x}_{0}$ with eigenvalue $\mathbf{x}$, i.e.,

$$
\left.\Pi_{\mathbf{x}}=\sum_{\epsilon= \pm} \sum_{s=0, \pm 1} \mid A_{\mathbf{x}}^{(\epsilon, s)}\right)\left(A_{\mathbf{x}}^{(\epsilon, s)} \mid,\right.
$$

$\|\cdot\|^{2}:=((\cdot, \cdot))$ is the square of the norm of $\mathcal{H}$, and we assume $\|A\|=1$. Substituting (195) in (194) and making use of (150) and (151), we have $P_{V}=\sum_{\epsilon= \pm} \sum_{s=0, \pm 1} \int_{V} d^{3} \mathbf{x}|f(\epsilon, s, \mathbf{x})|^{2}$. Therefore, in light of (168), (82), and (83), the probability density is given by

$$
\varrho\left(x_{0}^{0}, \mathbf{x}\right):=\sum_{\epsilon= \pm} \sum_{s=0, \pm 1}|f(\epsilon, s, \mathbf{x})|^{2}=\frac{\kappa}{2 \mathcal{M}}\left\{\left|\mathfrak{U} \mathbf{A}\left(x_{0}^{0}, \mathbf{x}\right)\right|^{2}+\left|\mathfrak{U} D^{-1 / 2} \dot{\mathbf{A}}\left(x_{0}^{0}, \mathbf{x}\right)\right|^{2}\right\} .
$$

For a position measurement to be made at time $t=x^{0} / c$, we have the probability density

$$
\varrho\left(x^{0}, \mathbf{x}\right)=\frac{\kappa}{2 \mathcal{M}}\left\{\left|\mathfrak{U} \mathbf{A}\left(x^{0}, \mathbf{x}\right)\right|^{2}+\left|\mathfrak{U} D^{-1 / 2} \dot{\mathbf{A}}\left(x^{0}, \mathbf{x}\right)\right|^{2}\right\}=\frac{\kappa}{2 \mathcal{M}}\left\{|\mathfrak{U} \mathbf{A}(x)|^{2}+\left|\mathfrak{U} \mathbf{A}_{c}(x)\right|^{2}\right\} .
$$

We can use the method discussed in Sec. V to introduce a current density $\mathcal{J}^{\mu}$ such that $\mathcal{J}^{0}$ $=\varrho$. (See also Ref. 50.) This probability current density turns out to have the form

$$
\mathcal{J}^{\mu}(x)=\frac{\kappa}{2 \mathcal{M}} \mathfrak{R}\left\{\mathfrak{U} \mathbf{A}(x)^{*} \cdot\left(D^{-1} \partial^{\mu} \mathfrak{U} \dot{\mathbf{A}}(x)\right)+\mathfrak{U} \mathbf{A}_{c}(x)^{*} \cdot\left(D^{-1} \partial^{\mu} \mathfrak{U} \dot{\mathbf{A}}_{c}(x)\right)\right\}+\Upsilon^{\mu}(x),
$$

where $\mathfrak{R}$ means "the real part of", $\Upsilon^{0}(x)=0$ and, for all $i \in\{1,2,3\}$,

$$
\begin{aligned}
\Upsilon^{i}(x)= & \frac{\kappa}{2 \mathcal{M}} \mathfrak{R}\left\{(\mathfrak{U} \mathbf{A}(x))^{i *}\left[\boldsymbol{\nabla} \cdot \frac{D^{-1 / 2}}{D^{1 / 2}+\mathcal{M}} \mathfrak{U} \dot{\mathbf{A}}(x)\right]-\left[\mathfrak{U} \mathbf{A}(x)^{i *} \cdot \nabla\right]\left(\frac{D^{-1 / 2}}{D^{1 / 2}+\mathcal{M}} \mathfrak{U} \dot{\mathbf{A}}(x)\right)^{i}\right. \\
& \left.+\left(\mathfrak{U} \mathbf{A}_{c}(x)\right)^{i *}\left[\boldsymbol{\nabla} \cdot \frac{D^{-1 / 2}}{D^{1 / 2}+\mathcal{M}} \mathfrak{U} \dot{\mathbf{A}}_{c}(x)\right]-\left[\mathfrak{U} \mathbf{A}_{c}(x)^{i *} \cdot \boldsymbol{\nabla}\right]\left(\frac{D^{-1 / 2}}{D^{1 / 2}+\mathcal{M}} \mathfrak{U} \dot{\mathbf{A}}_{c}(x)\right)^{i}\right\} .
\end{aligned}
$$

One can easily show that $\mathcal{J}^{\mu}(x)$ is neither a four-vector nor a conserved current density.

Although the above discussion is based on a particular choice for the parameters $\{\mathfrak{a}\}$, namely, $\{\mathfrak{a}\}=\{1\}$, it is generally valid. This is because the position wave functions $f$ and the corresponding probability densities do not depend on the parameters $\{\mathfrak{a}\}$. Therefore, if we are to compute the probability density $\varrho_{\{\mathfrak{a}\}}$ of the spatial localization of a Proca field $A \in \mathcal{H}_{\{\mathfrak{a}\}}$ with the position operator being identified with $\mathbf{x}_{0\{\mathfrak{a}\}}$ for $\{\mathfrak{a}\} \neq\{1\}$, we have, for a measurement made at $t_{0}=x_{0}^{0} / \mathrm{c}$,

$$
\varrho_{\{\mathfrak{a}\}}\left(x_{0}^{0}, \mathbf{x}\right)=\frac{\kappa}{2 \mathcal{M}}\left\{\left|\mathfrak{U} \mathbf{A}_{\{\mathfrak{a}\}}^{\prime}\left(x_{0}^{0}, \mathbf{x}\right)\right|^{2}+\left|\mathfrak{U} D^{-1 / 2} \dot{\mathbf{A}}_{\{\mathfrak{a}\}}^{\prime}\left(x_{0}^{0}, \mathbf{x}\right)\right|^{2}\right\},
$$

where $\mathbf{A}_{\{\mathfrak{a}\}}^{\prime}:=U_{\{\mathfrak{a}\}}^{-1} \mathbf{A}$ and $U_{\{\mathfrak{a}\}}: \mathcal{H} \rightarrow \mathcal{H}_{\{\mathfrak{a}\}}$ is given by (153). We can compute $\mathbf{A}_{\{\mathfrak{a}\}}^{\prime}$ using (65), (120), and (153) and use the result to obtain

$$
\begin{aligned}
\varrho_{\{\mathfrak{a}\}}(x)= & \frac{\kappa}{4 \mathcal{M}}\left\{\left|\mathfrak{U}_{+,+} \mathbf{A}(x)\right|^{2}+\left|\mathfrak{U}_{-,+} \mathbf{A}(x)\right|^{2}+\left|\mathfrak{U}_{+,+} \mathbf{A}_{c}(x)\right|^{2}+\left|\mathfrak{U}_{-,+} \mathbf{A}_{c}(x)\right|^{2}\right. \\
& \left.+2 \mathfrak{R}\left[\left(\mathfrak{U}_{+,+} \mathbf{A}(x)\right)^{*} \cdot \mathfrak{U}_{+,+} \mathbf{A}_{c}(x)-\left(\mathfrak{U}_{-,+} \mathbf{A}(x)\right)^{*} \cdot \mathfrak{U}_{-,+} \mathbf{A}_{c}(x)\right]\right\} .
\end{aligned}
$$

Again we can use the method of Sec. V to compute a probability current density $\mathcal{J}_{\{\mathfrak{a}\}}^{\mu}$ such that $\mathcal{J}_{\{a\}}^{0}=\varrho_{\{\mathfrak{a}\}}$. This leads to a complicated expression that we do not include here. Similarly to $\mathcal{J}^{\mu}$, we expect $\mathcal{J}_{\{\mathfrak{a}\}}^{\mu}$ to be neither covariant nor conserved. 
The nonconservation (noncovariance) of the probability current density $\mathcal{J}_{\{\mathfrak{a}\}}^{\mu}$ raises the issue of the nonconservation (frame dependence) of the total probability,

$$
\mathscr{P}_{\{\mathfrak{a}\}}:=\int_{\mathbb{R}^{3}} d^{3} \mathbf{x} \varrho_{\{\mathfrak{a}\}}\left(x^{0}, \mathbf{x}\right) .
$$

This would certainly be unacceptable. The situation is analogous to that of the KG fields. $\mathscr{P}_{\{\mathrm{a}\}}$ is indeed a frame-independent conserved quantity, thanks to the covariance and conservation of the current density $J_{\{\mathfrak{a}\}}^{\mu}$ and the identity

$$
\int_{\mathbb{R}^{3}} d^{3} \mathbf{x} \varrho_{\{\mathfrak{a}\}}\left(x^{0}, \mathbf{x}\right)=\int_{\mathbb{R}^{3}} d^{3} \mathbf{x} J_{\{\mathfrak{a}\}}^{0}\left(x^{0}, \mathbf{x}\right),
$$

which follows from (128) and (201) and the fact that $\mathfrak{U}_{\epsilon, \epsilon^{\prime}}$ are self-adjoint operators acting in $\tilde{\mathcal{H}}$.

Combining (202) and (203), we have

$$
\mathscr{P}_{\{a\}}=\int_{\mathbb{R}^{3}} d^{3} \mathbf{x} J_{\{a\}}^{0}\left(x^{0}, \mathbf{x}\right) .
$$

This relation implies that although the probability density $\varrho_{\{\mathfrak{a}\}}$ is not the zero component of a conserved four-vector current density, its integral over the whole space that yields the total probability (202) is nevertheless conserved. Furthermore, this global conservation law stems from a local conservation law, i.e., a continuity equation for a four-vector current density, namely, $J_{\{\mathfrak{a}\}}^{\mu}$.

\section{GAUGE SYMMETRY ASSOCIATED WITH THE CONSERVATION OF THE TOTAL PROBABILITY}

In this section we explore a global gauge symmetry that supports the conservation of the total probability or its local realization as the conservation of the current density $J_{\{\mathfrak{a}\}}^{\mu}$. To determine the nature of this symmetry, we recall that the conserved charge associated with any conserved current is the generator of the corresponding infinitesimal gauge transformations. ${ }^{51}$ We use the Hamiltonian formulation to obtain these transformations. The procedure we follow mimics the one presented in Ref. 6 for the KG fields.

The Lagrangian $L$ for a Proca field $A$ and the corresponding canonical momenta $\Pi(\mathbf{x}), \bar{\Pi}(\mathbf{x})$ associated with $A(\mathbf{x}):=A\left(x^{0}, \mathbf{x}\right)$ and $A^{*}\left(x^{0}, \mathbf{x}\right)$ are, respectively, given by ${ }^{3}$

$$
\begin{gathered}
L:=-\int_{\mathbb{R}^{3}} d^{3} \mathbf{x}\left\{\frac{1}{2} F_{\mu \nu}(\mathbf{x})^{*} F^{\mu \nu}(\mathbf{x})+\mathcal{M}^{2} A_{\mu}(\mathbf{x})^{*} A^{\mu}(\mathbf{x})\right\}, \\
\Pi^{0}(\mathbf{x}):=\frac{\delta L}{\delta \dot{A}_{0}(\mathbf{x})}=0, \quad \Pi^{i}(\mathbf{x}):=\frac{\delta L}{\delta \dot{A}_{i}(\mathbf{x})}=-F^{0 i}(\mathbf{x})^{*}=-E^{i}(\mathbf{x})^{*}, \\
\bar{\Pi}^{0}(\mathbf{x}):=\frac{\delta L}{\delta \dot{A}_{0}(\mathbf{x})^{*}}=0, \quad \bar{\Pi}^{i}(\mathbf{x}):=\frac{\delta L}{\delta \dot{A}_{i}(\mathbf{x})^{*}}=-F^{0 i}(\mathbf{x})=-E^{i}(\mathbf{x}) .
\end{gathered}
$$

Throughout this section we suppress the $x^{0}$-dependence of the fields for simplicity. The fact that $\Pi^{0}$ and $\bar{\Pi}^{0}$ vanish shows that the Proca system is a constrained system. There are two primary constraints

$$
\Phi_{1}:=\Pi^{0}(x) \approx 0, \quad \Phi_{2}:=\bar{\Pi}^{0}(x) \approx 0 .
$$

Following Dirac's notation, ${ }^{52}$ we write the constraints as weak equations with the weak equality 
symbol " $\approx . "$ Solving for the velocities $\dot{A}_{i}$ and $\dot{A}_{i}^{*}$ in (206) and (207), and using the Hamiltonian

$$
\begin{aligned}
H_{0}:= & \int_{\mathbb{R}^{3}} d^{3} \mathbf{x}\left\{\Pi_{i}(\mathbf{x}) \bar{\Pi}^{i}(\mathbf{x})-\Pi^{i}(\mathbf{x}) \partial_{i} A_{0}(\mathbf{x})-\bar{\Pi}^{i}(\mathbf{x}) \partial_{i} A_{0}(\mathbf{x})^{*}+\mathcal{M}^{2} A_{0}(\mathbf{x}) A^{0}(\mathbf{x})^{*}+\mathcal{M}^{2} A_{i}(\mathbf{x}) A^{i}(\mathbf{x})^{*}\right. \\
& \left.+\partial_{i} A_{j}(\mathbf{x}) \partial^{i} A^{j}(\mathbf{x})^{*}-\partial_{i} A_{j}(\mathbf{x}) \partial^{j} A^{i}(\mathbf{x})^{*}\right\},
\end{aligned}
$$

we obtain the so-called total Hamiltonian: ${ }^{52} H_{T}:=H_{0}+u^{j} \Phi_{j}$, where $u^{j}, j=1,2$ are two unknown coefficients. Using the Poisson bracket

$$
\{\mathcal{F}, \mathcal{G}\}_{P}:=\int_{\mathbb{R}^{3}} d^{3} \mathbf{x}\left[\frac{\delta \mathcal{F}}{\delta A_{\mu}(\mathbf{x})} \frac{\delta \mathcal{G}}{\delta \Pi^{\mu}(\mathbf{x})}-\frac{\delta \mathcal{G}}{\delta A_{\mu}(\mathbf{x})} \frac{\delta \mathcal{F}}{\delta \Pi^{\mu}(\mathbf{x})}+\frac{\delta \mathcal{F}}{\delta A_{\mu}(\mathbf{x})^{*}} \frac{\delta \mathcal{G}}{\delta \bar{\Pi}^{\mu}(\mathbf{x})}-\frac{\delta \mathcal{G}}{\delta A_{\mu}(\mathbf{x})^{*}} \frac{\delta \mathcal{F}}{\delta \bar{\Pi}^{\mu}(\mathbf{x})}\right]
$$

of the observables $\mathcal{F}$ and $\mathcal{G}$, we can easily show that the dynamical consistency of the primary constraints (208), i.e., $\dot{\Phi}_{j}=\left\{\Phi_{j}, H_{T}\right\}_{P} \approx 0$, results in the following secondary constraints:

$$
\Phi_{3}:=\mathcal{M}^{2} A_{0}(\mathbf{x})+\partial_{i} \bar{\Pi}^{i}(\mathbf{x}) \approx 0, \quad \Phi_{4}:=\mathcal{M}^{2} A_{0}(\mathbf{x})^{*}+\partial_{i} \Pi^{i}(\mathbf{x}) \approx 0,
$$

and that there is no other secondary constraint.

It is not difficult to show that the matrix $\mathscr{C}_{j j^{\prime}}(\mathbf{y}, \mathbf{z}):=\left\{\Phi_{j}(\mathbf{y}), \Phi_{j^{\prime}}(\mathbf{z})\right\}_{P}$ is nonsingular. Hence we have a theory with four second-class constraints and can apply Dirac's canonical quantization that uses the Dirac bracket, ${ }^{52}$

$$
\{\mathcal{F}, \mathcal{G}\}_{D}:=\{\mathcal{F}, \mathcal{G}\}_{P}-\int_{\mathbb{R}^{3}} d^{3} \mathbf{y} \int_{\mathbb{R}^{3}} d^{3} \mathbf{z}\left\{\mathcal{F}, \Phi_{j}(\mathbf{y})\right\}_{P} \mathscr{C}^{j j^{\prime}}(\mathbf{y}, \mathbf{z})\left\{\Phi_{j^{\prime}}(\mathbf{z}), \mathcal{G}\right\}_{P}
$$

where $\mathscr{C}^{j j^{\prime}}(\mathbf{y}, \mathbf{z})$ is the inverse of $\mathscr{C}_{j j^{\prime}}(\mathbf{y}, \mathbf{z})$. Computing the latter and its inverse, we find $\mathscr{C}^{j j^{\prime}}(\mathbf{y}, \mathbf{z}):=\tilde{\mathscr{C}}^{j j^{\prime}} \delta^{3}(\mathbf{y}-\mathbf{z})$, and

$$
\{\mathcal{F}, \mathcal{G}\}_{D}=\{\mathcal{F}, \mathcal{G}\}_{P}-\int_{\mathbb{R}^{3}} d^{3} \mathbf{y}\left\{\mathcal{F}, \Phi_{j}(\mathbf{y})\right\}_{P} \tilde{\mathscr{C}}^{j j^{\prime}}\left\{\Phi_{j^{\prime}}(\mathbf{y}), \mathcal{G}\right\}_{P},
$$

where $\tilde{\mathscr{C}}^{13}=\tilde{\mathscr{C}}^{24}=-\tilde{\mathscr{C}}^{31}=-\tilde{\mathscr{C}}^{42}=\mathcal{M}^{-2}$ and $\tilde{\mathscr{C}}^{j j^{\prime}}=0$ for other $j$ 's and $j^{\prime}$ 's. Further details of the constraint quantization of Proca system can be found in Ref. 53-55.

In terms of the canonical phase space variables $(A, \Pi)$ and $\left(A^{*}, \bar{\Pi}\right)$, the total probability (204) takes the form

$$
\begin{aligned}
\mathscr{P}_{\{\mathfrak{a}\}}= & \frac{\kappa}{2 \mathcal{M}} \int_{\mathbb{R}^{3}} d^{3} \mathbf{x}\left\{\Pi^{i}(\mathbf{x})\left[\Theta_{+, 0} D^{-1 / 2} \dot{\mathbf{A}}(\mathbf{x})\right]_{i}-A_{i}(\mathbf{x})^{*}\left[\Theta_{+, 0} D^{-1 / 2} \dot{\bar{\Pi}}(\mathbf{x})\right]^{i}\right. \\
& \left.+i\left(\Pi^{i}(\mathbf{x})\left[\Theta_{+, 0} \mathbf{A}(\mathbf{x})\right]_{i}-A_{i}(\mathbf{x})^{*}\left[\Theta_{+, 0} \overline{\mathbf{\Pi}}(\mathbf{x})\right]^{i}\right)\right\}
\end{aligned}
$$

where we have made use of (128), (206), and (207). Now, we can obtain the infinitesimal symmetry transformation,

$$
A \rightarrow A+\delta A,
$$

generated by $\mathscr{P}_{\{a\}}$ using

$$
\delta A(\mathbf{x})=\left\{A(\mathbf{x}), \mathscr{P}_{\{\mathfrak{a}\}}\right\}_{D} \delta \phi,
$$

where $\delta \phi$ is an infinitesimal real parameter. In view of (206), (207), (210), (213), (216), (29), (33), and (82) we have 


$$
\begin{gathered}
\delta A^{0}(\mathbf{x})=-i \delta \theta\left\{L_{+}^{0} \mathrm{C}+L_{-}^{0}\right\} A^{0}(\mathbf{x}), \\
\delta \mathbf{A}(\mathbf{x})=-i \delta \theta\left\{\Theta_{+, 0} \mathrm{C}+\Theta_{-, 0}\right\} \mathbf{A}(\mathbf{x}),
\end{gathered}
$$

where $\delta \theta:=\kappa \delta \phi /(2 \mathcal{M})$ and $\Theta_{ \pm, 0}$ are given by (99). We may employ (100) to express (217) and (218) as

$$
\delta A(\mathbf{x})=-i \delta \theta\left\{\vartheta_{+, 0} \mathrm{C}+\vartheta_{-, 0}\right\} A(\mathbf{x}),
$$

where $\vartheta_{\epsilon, 0}: \mathcal{H}_{\{a\}} \rightarrow \mathcal{H}_{\{a\}}$ is defined by

$$
\vartheta_{\epsilon, 0}:=\left[L_{\epsilon}^{+}-L_{\epsilon}^{0}\right] \mathfrak{H}^{2}+L_{\epsilon}^{-} \mathfrak{H}+L_{\epsilon}^{0} .
$$

In view of (219), the symmetry transformations (215) are generated by the operator $\vartheta_{+, 0} \mathrm{C}$ $+\vartheta_{-, 0}$. We can easily exponentiate the latter to obtain the corresponding noninfinitesimal symmetry transformations,

$$
A \rightarrow e^{-i \theta\left(\vartheta_{+, 0} \mathrm{C}+\vartheta_{-, 0}\right)} A,
$$

where $\theta \in \mathbb{R}$ is arbitrary. In terms of the $(\epsilon, h)$-components $A_{\epsilon, h}$ of $A,(221)$ takes the form

$$
A=\sum_{\epsilon= \pm} \sum_{h=0, \pm 1} A_{\epsilon, h} \rightarrow \sum_{\epsilon= \pm} \sum_{h=0, \pm 1} e^{-i \epsilon \mathfrak{a}_{\epsilon, h}} A_{\epsilon, h}
$$

where we have made use of $C^{2}=1$ and $\mathfrak{H}^{3}=\mathfrak{H}$.

Similarly to its spin-zero counterpart, ${ }^{6}$ as seen from (221) and (222), the gauge group $G_{\{\mathfrak{a}\}}$ associated with these transformations is a one-dimensional connected Abelian Lie group. Therefore, it is isomorphic to either of $U(1)$ or $\mathbb{R}^{+}$, the latter being the noncompact multiplicative group of positive real numbers. ${ }^{56}$ (Here we identify the gauge group with its connected component that includes the identity and is obtained by exponentiating the generator $\vartheta_{+, 0} \mathrm{C}+\vartheta_{-, 0}$.)

We can construct a faithful representation of the group $G_{\{\mathfrak{a}\}}$ using the six-component representation $A=\left(A_{+,+1}, A_{+,-1}, A_{+, 0}, A_{-,+1}, A_{-,-1}, A_{-, 0}\right)^{T}$, where $\mathrm{C}$ and $\mathfrak{H}$ are, respectively, represented by $\Sigma_{3}$ and $\Sigma_{12}$. In this representation a typical element of $G_{\{\mathfrak{a}\}}$ takes the form

$$
g_{a}(\theta):=\operatorname{diag}\left(e^{-i \mathfrak{a}_{+,+1} \theta}, e^{-i \mathfrak{a}_{+,-1} \theta}, e^{-i \mathfrak{a}_{+, 0} \theta}, e^{i \mathfrak{a}_{-,+1} \theta}, e^{i \mathfrak{a}_{-,-1} \theta}, e^{i \mathfrak{a}_{-, 0} \theta}\right) .
$$

This expression suggests that the gauge group $G_{\{a\}}$ is a subgroup of $U(1) \otimes^{6}$, the latter being the direct product of six copies of $U(1)$. It is not difficult to show that $G_{\{\mathfrak{a}\}}$ is a compact subgroup of this group and consequently isomorphic to $U(1)$ if and only if all the parameters $\mathfrak{a}_{\epsilon, h}$ are rational numbers, otherwise $G_{\{\mathfrak{a}\}}$ is isomorphic to $\mathbb{R}^{+}$.

Clearly, the $G_{\{\mathfrak{a}\}}$ gauge symmetry associated with the conservation of the total probability is a global gauge symmetry. Similarly to its spin-zero counterpart, ${ }^{6}$ the local analog of this global gauge symmetry is different from the usual local Yang-Mills-type gauge symmetries.

\section{CONCLUSION AND DISCUSSION}

In this article we have used the methods of PHQM to devise a complete formulation of the RQM of the Proca fields that does not involve restricting to the positive-energy solutions of the Proca equation. In particular, we have constructed the most general physically admissible inner product $((\cdot, \cdot))_{\{\mathfrak{a}\}}$ on the solution space of the Proca equation. Up to a trivial scaling, this inner product involves five real parameters that we collectively denote by $\mathfrak{a}$. For all the values of $\mathfrak{a}$ the inner product $((\cdot, \cdot))_{\{a\}}$ is positive-definite and relativistically invariant. It also renders the generator of the time-translations, i.e., the Hamiltonian h, and the helicity operator self-adjoint. The quantum system associated with the Proca fields may be represented by a Hilbert space $\mathcal{H}_{\{a\}}$ defined by the inner product $((\cdot, \cdot))_{\{\mathfrak{a}\}}$ and the Hamiltonian $h$. Using the unitary equivalence of this covariant representation with that of the noncovariant Foldy representation, which arises quite 
naturally in our formulation, we constructed relativistic position, momentum, angular momentum, spin and helicity operators acting in $\mathcal{H}_{\{\mathfrak{a}\}}$, and the localized states of the Proca field. Furthermore, we introduced the position wave functions and used them to construct a probability current density which turned out to be neither conserved nor covariant. We resolved the apparent inconsistency of this observation with the physical requirement of the conservation and frame independence of the total probability using a conserved and covariant current density. The global conservation of the total probability is supported by the local conservation of this current density. The latter is linked to a previously unnoticed global gauge symmetry of the Proca field with an Abelian gauge group.

In Ref. 29, the authors have also attempted to give a spin-one generalization of our treatment of the KG fields. However, because of various self-imposed restrictions they obtain a oneparameter subfamily of the inner products $((\cdot, \cdot))_{\{\mathfrak{a}\}}$. This is related to the fact that the authors of Ref. 29 used the special Foldy transformation (65) [see Eq. (35) of Ref. 29] to find the metric operator acting in the Hilbert space $\mathcal{H}^{\prime}$. (The treatment of Ref. 29 has some minor errors. For example, the system $\left\{\Psi_{\epsilon, h}(\mathbf{k}), \Phi_{\epsilon, h}(\mathbf{k})\right\}$ used in Ref. 29 to construct the metric operator is actually not biorthonormal, as claimed by the authors. Fortunately, this type of errors could be absorbed in the unknown parameters of the metric operator and do not affect the final result.) Furthermore, in trying to impose the condition of the Lorentz-invariance of the metric operator, they transform the operator $\tilde{\eta}_{+}$to a metric operator acting in the Foldy representation and demand that the latter commutes with the generators of the Poincare group in this representation. This does not seem to be well justified because as seen from (40), the Hilbert space of the Foldy representation is just the direct sum of two copies of $\tilde{\mathcal{H}}$. Hence, the metric operator associated with this representation is just the identity operator, not the one given by Eq. (39) of Ref. 29. As seen from (117), this is the Foldy transformation which depends on the choice of the unknown parameters appearing in the operator $\widetilde{\eta}_{+}$, not the metric operator of the Foldy representation. We would also like to stress that the analysis of Ref. 29 does not include the construction of the observables of the system or any treatment of its physical aspects such as the notorious problem of the probabilistic interpretation of the QM of Proca fields.

Apart from the historical importance of the subject, the present work is mainly motivated by the close analogy of the Proca and Maxwell fields. Performing the zero-mass limit of our results in an appropriate manner should lead to a consistent quantum mechanical treatment of individual photons. As it is to be expected, there are subtleties in performing this limit. Nevertheless, we have been able to make some progress toward solving the problem of the construction of the Hilbert space and observables for the photon. We plan to report the results in a separate article.

\section{ACKNOWLEDGMENTS}

F.Z. wishes to acknowledge the hospitality of Koç University during his visits. This project was supported by Turkish Academy of Sciencs (TÜBA).

${ }^{1}$ E. P. Wigner, Ann. Math. 40, 149 (1939).

${ }^{2}$ V. Bargemann and E. P. Wigner, Proc. Natl. Acad. Sci. U.S.A. 34, 211 (1948).

${ }^{3}$ S. Weinberg, The Quantum Theory of Fields (Cambridge University Press, Cambridge, 1995).

${ }^{4}$ A. Peres and D. R. Terno, Rev. Mod. Phys. 76, 93 (2004).

${ }^{5}$ A. Mostafazadeh and A. Batal, J. Phys. A 37, 11645 (2004); A. Mostafazadeh, ibid. 38, 6557 (2005).

${ }^{6}$ A. Mostafazadeh and F. Zamani, Ann. Phys. (N.Y.) 321, 2183 (2006).

${ }^{7}$ A. Proca, J. Phys. Radium 7, 347 (1936); see also D. N. Poenaru and A. Calboreanu, Europhys. News 37, 24 (2006).

${ }^{8}$ W. Pauli and V. Weisskopf, Helv. Phys. Acta 7, 709 (1934).

${ }^{9}$ L.-C. Tu, J. Luo, and G. T. Gillies, Rep. Prog. Phys. 68, 77 (2005).

${ }^{10}$ M. Hawton, Phys. Rev. A 59, 954 (1999); 59, 3223 (1999); 75, 062107 (2007); M. Hawton and W. E. Baylis, ibid. 64, 052103 (2001)

${ }^{11}$ B. Vijayalakshmi, M. Seetharaman, and P. M. Mathews, J. Phys. A 12, 665 (1979).

${ }^{12}$ A. J. Silenko, e-print arXiv:hep-th/0404074.

${ }^{13}$ N. Kemmer, Proc. R. Soc. London, Ser. A 177, 9 (1939).

${ }^{14}$ S. Weinberg, Phys. Rev. 133, B1318 (1964); S. Shay and R. H. Good, Jr., Phys. Rev. 179, 1410 (1969).

${ }^{15}$ M. Taketani and S. Sakata, Proc. Phys. Math. Soc. Jpn. 22, 757 (1940), reprinted as , Suppl. Prog. Theor. Phys. 1, 84 (1955).

${ }^{16}$ K. M. Case, Phys. Rev. 95, 1323 (1954). 
${ }^{17}$ L. L. Foldy, Phys. Rev. 102, 568 (1956).

${ }^{18}$ W. Pauli, Rev. Mod. Phys. 15, 175 (1943); S. N. Gupta, Proc. Phys. Soc. London 63, 681 (1950); K. Bleuler, Helv. Phys. Acta 23, 567 (1950); E. C. G. Sudarshan, Phys. Rev. 123, 2183 (1961); T. D. Lee and G. C. Wick, Nucl. Phys. B 9, 209 (1969); N. Nakanishi, Suppl. Prog. Theor. Phys. 51, 1 (1972); A. Mostafazadeh, Czech. J. Phys. 53, 1079 (2003); e-print arXiv:quant-ph/0308028.

${ }^{19}$ M. H. L. Pryce, Proc. R. Soc. London, Ser. A 195, 62 (1948); T. F. Jordan and N. Mukunda, Phys. Rev. 132, 1842 (1963); H. Bacry, J. Math. Phys. 5, 109 (1964); R. A. Berg, ibid. 6, 34 (1965); A. Sankaranarayanan and R. H. Good, Jr., Phys. Rev. 140, B509 (1965); J. E. Johnson, Phys. Rev. 181, 1755 (1969); T. F. Jordan, J. Math. Phys. 21, 2028 (1980).

${ }^{20}$ J. A. de Azcárraga, L. Oliver, and J. Pascual, Phys. Rev. D 8, 4375 (1973).

${ }^{21}$ T. D. Newton and E. P. Wigner, Rev. Mod. Phys. 21, 400 (1949).

${ }^{22}$ A. S. Wightman, Rev. Mod. Phys. 34, 845 (1962); T. O. Philips, Phys. Rev. 136, B893 (1964); A. J. Kállnay, Phys. Rev. D 1, 1092 (1970); A. J. Kállnay and P. L. Torres, ibid. 9, 1670 (1974).

${ }^{23}$ J. Daicic and N. E. Frankel, Prog. Theor. Phys. 88, 1 (1992); , J. Phys. A 26, 1397 (1993); V. K. Mishra, S. Hama, B. C. Clark, R. E. Kozack, R. L. Mercer, and L. Ray, Phys. Rev. C 43, 801 (1991); Y. Nedjadi and R. C. Barrett, J. Math. Phys. 35, 4517 (1994); Y. Sucu and N. Ünal, Eur. Phys. J. C 44, 287 (2005); A. Zecca, Gen. Relativ. Gravit. 38, 837 (2006). ${ }^{24}$ V. G. Bagrov, A. Y. Trifonov, and A. A. Yevseyevich, Class. Quantum Grav. 9, 533 (1992).

${ }^{25}$ A. Mostafazadeh, Class. Quantum Grav. 20, 155 (2003).

${ }^{26}$ A. Mostafazadeh, Ann. Phys. (N.Y.) 309, 1 (2004).

${ }^{27}$ A. Mostafazadeh, Int. J. Mod. Phys. A 21, 2553 (2006).

${ }^{28}$ A. Mostafazadeh and F. Zamani, Ann. Phys. (N.Y.) 321, 2210 (2006).

${ }^{29}$ V. Jakubský and J. Smejkal, Czech. J. Phys. 56, 985 (2006).

${ }^{30}$ A. Mostafazadeh, Czech. J. Phys. 53, 1079 (2003); , J. Phys. A 38, 3213 (2005).

${ }^{31}$ C. M. Bender and S. Boettcher, Phys. Rev. Lett. 80, 5243 (1998); C. M. Bender, S. Boettcher, and P. N. Meisenger, J. Math. Phys. 40, 2201 (1999).

${ }^{32}$ A. Mostafazadeh, J. Math. Phys. 43, 2814 (2002).

${ }^{33}$ A. Mostafazadeh, J. Math. Phys. 43, 205 (2002).

${ }^{34}$ F. G. Scholtz, H. B. Geyer, and F. J. W. Hahne, Ann. Phys. 213, 74 (1992).

${ }^{35}$ A. Mostafazadeh, J. Math. Phys. 44, 974 (2003).

${ }^{36}$ C. M. Bender, D. C. Brody, and H. F. Jones, Phys. Rev. Lett. 89, 270401 (2002).

${ }^{37}$ Z. Ahmed and S. R. Jain, J. Phys. A 36, 9711 (2003); Z. Ahmed, Phys. Lett. A 310, 139 (2003).

${ }^{38}$ A. Mostafazadeh, J. Math. Phys. 43, 3944 (2002).

${ }^{39}$ A. Mostafazadeh, Nucl. Phys. B 640, 419 (2002).

${ }^{40}$ S. A. Fulling, Aspects of Quantum Field Theory in Curved Space-Time (Cambridge University Press, Cambridge, 1989).

${ }^{41}$ W. Greiner and J. Reinhardt, Field Quantization (Springer, Berlin, 1996).

${ }^{42}$ W. Greiner and B. Müller, Quantum Mechanics: Symmetries (Springer, Berlin, 1994).

${ }^{43}$ W. Greiner, Relativistic Quantum Mechanics (Springer, Berlin, 1994).

${ }^{44}$ K. Haller, Phys. Rev. D 8, 1796 (1973).

${ }^{45}$ M. Znojil and H. B. Geyer, Phys. Lett. B 640, 52 (2006).

${ }^{46}$ F. J. Ynduráin, Relativistic Quantum Mechanics and Introduction to Field Theory (Springer, Berlin, 1996).

${ }^{47}$ T. Kato, Perturbation Theory for Linear Operators (Springer, Berlin, 1995).

${ }^{48}$ Sz. Farkas, Z. Kurucz, and M. Weiner, Int. J. Theor. Phys. 41, 79 (2002).

${ }^{49}$ G. C. Wick, A. S. Wightman, and E. P. Wigner, Phys. Rev. 88, 101 (1952).

${ }^{50}$ B. Rosenstein and L. P. Horwitz, J. Phys. A 18, 2115 (1985).

${ }^{51}$ C. Itzykson and J.-B. Zuber, Quantum Field Theory (McGraw-Hill, New York, 1980).

${ }^{52}$ P. A. M. Dirac, The Principles of Quantum Mechanics (Oxford University Press, Oxford, 1958).

${ }^{53}$ D. M. Gitman and I. V. Tyutin, Quantization of Fields with Constraints (Springer, Berlin, 1990); M. Henneaux and C. Teitelboim, Quantization of Gauge Systems (Princeton University Press, Princeton, 1992).

${ }^{54}$ Y. W. Kim, M. I. Park, Y. J. Park, and S. J. Yoon, Int. J. Mod. Phys. A 12, 4217 (1997).

${ }^{55}$ J. Smejkal, V. Jakubský, and M. Znojil, J. Phys. Stud. 11, 45 (2007).

${ }^{56}$ T. Bröcker and T. T. Dieck, Representations of Compact Lie Groups (Springer, Berlin, 1985). 